Commun. Math. Phys. 146, 139-204 (1992)

Communications in

Mathematical

Physics

(C) Springer-Verlag 1992

\title{
Mathematical Models with Exact Renormalization for Turbulent Transport, II: Fractal Interfaces, Non-Gaussian Statistics and the Sweeping Effect
}

\author{
Marco Avellaneda ${ }^{1 \star}$ and Andrew Majda ${ }^{2 \star \star}$ \\ ${ }^{1}$ Courant Institute, New York University, New York, NY 10012, USA \\ 2 Department of Mathematics and Program in Appl. \& Computa. Math., Princeton University, \\ Princeton, NJ 08544, USA
}

Received June 6, 1991

\begin{abstract}
This paper continues the study of a model for turbulent transport with an exact renormalization theory which has recently been proposed and developed by the authors. Three important topics are analyzed with complete mathematical rigor for this model: (1) Renormalized higher order statistics of a passively advected scalar such as the pair distance distribution and the fractal dimension of interfaces, (2) the effect of non-Gaussian turbulent velocity statistics on renormalization theory, (3) the "sweeping" effect of additional large scale mean velocities. A special emphasis is placed on renormalization theory in the vicinity of the value of the analogue of the Kolmogorov-spectrum in the model. In the authors' earlier paper, it was established that the Kolmogorov value is at a phase transition boundary in the exact renormalization theory. It is found here that the qualitative model, despite its simplicity contains, in the vicinity of the Kolmogorov value, a remarkable amount of the qualitative behavior of turbulent transport which has been uncovered in recent experiments and proposed in phenomenological theories. In particular, the Richardson 4/3-law for pair dispersion and interfaces with fractal dimension defect of $2 / 3$ occur in the model rigorously as limits when the Kolmogorov spectrum is approached as a limit from one side of the phase transition boundary; alternative corrections to the Richardson law with the same form as those proposed heuristically in the recent literature and interfaces with fractal dimension defect $1 / 3$, occur in the model when the Kolmogorov spectrum is approached from the other side of the phase transition. It is very interesting that fractal dimension defects of roughly the value either $1 / 3$ or $2 / 3$ for level sets and interfaces of passive scalars have been ubiquitous in recent turbulence experiments. As regards non-Gaussian
\end{abstract}

* Research partially supported by NSF-DMS-9005799, ARO-DAAL03-89-K-0039 and AFOSR 90-0090

$\star \star$ Research partially supported by grants NSF-DMS-90-01805, ARO-DAAL03-89-K-0013, and ONR-N00014-89-J-1014 
velocity statistics, a principle of "statistical universality" is established rigorously in the model so that the renormalization theory for eddy diffusivity coincides with the one presented in earlier work in the case of Gaussian velocity statistics. Finally, the authors show that the "sweeping effect" can significantly alter the renormalization theory in the model for suitable infrared divergent velocity statistics with steady or nearly steady velocity fields. However, it is proved here that the renormalization theory in the model in the vicinity of the Kolmogorov spectrum is Galilean invariant and insensitive to this sweeping effect of large scales.

\section{Introduction}

This paper concerns the long-time behavior of passively advected scalar quantities, such as heat or mass, evolving in a turbulent, incompressible velocity field. Passive scalar transport arises in a variety of physical situations, such as cloud dynamics, dispersion of pollutants in oceans and lakes and flow in porous media. The macroscopic description of the enhancement of dissipation rates in turbulent transport is a complex problem, due to the large fluctuations in the scalar fields caused by the turbulent flow. These problems are very difficult because the velocity involves a continuous range of excited space and/or time scales. An extremely important practical problem of this sort involves the computation of eddy diffusivities in fully developed turbulence. The goal of such theory is to assess the effects of the continuum of energetic smaller scales on the large scales through effective equations without resolving these effects explicitly. However, when non-dimensionalized on dissipation length scales, turbulent velocity fields exhibit strong infrared divergences exactly at the large scales where a theory of eddy diffusivity is needed (see [7]). Thus, these problems have been attacked through a wide variety of renormalized perturbation theories which mimic ideas from field theory and/or the renormalization group (R-N-G) from critical phenomena involving partial summation of divergent perturbation series $([10,13,15,16,19,24-26])$.

In developing theories for eddy diffusivity, the natural initial data involve only long wavelengths, i.e., the initial data has the form $T_{0}(\delta x)$ with $\delta \ll 1$. The first goal of an eddy diffusivity theory is to determine a nonlinear rescaling function $\rho(\delta)$ so that the ensemble average of solutions of the advection-diffusion equation

$$
\lim _{\delta \rightarrow 0}\left\langle T^{\delta}\left(\frac{x}{\delta}, \frac{t}{\rho^{2}}\right)\right\rangle=\bar{T}(x, t)
$$

satisfies an effective equation involving eddy diffusivity, i.e. enhanced dissipation. The issue of interest is then to characterize this effective evolution equation. The rescaling function, $\rho(\delta)$ is uniquely determined by the requirement that the above limit is nontrivial, i.e. neither identically zero nor merely $T_{0}(x)$, and serves to determine the critical time scale of important activity in the transport-diffusion process. The function $\rho(\delta)=\delta$ corresponds to the usual diffusive scaling. The behavior of the transport-diffusion process is superdiffusive provided $\rho(\delta)=\delta^{\theta}$ with $\theta<1$ since this corresponds to shorter time scales of nontrivial activity than the usual diffusive scalings while the behavior is super-ballistic if $\theta<\frac{1}{2}$ since this corresponds to motion which is faster than purely advective motion. 
Motivated by these important practical problems involving theories of eddy diffusivity, the authors recently introduced a model problem for renormalization of eddy diffusivity in turbulent transport involving simple shear flows with Gaussian statistics (see [1]). Furthermore, this model has an exactly solvable but remarkably complex renormalization theory for eddy diffusivity. The second-order statistics in these models involve two parameters, $\varepsilon$ and $z$ with $z \geqq 0$, where $\varepsilon$ measures the strength of the infrared divergence and $z$ measures the decorrelation time of long-wave portions of the statistical velocity spectrum (see [1] and (1.4)-(1.11) below). The rigorous renormalization theory for the exactly solvable model involves five phase regions in the $\varepsilon, z$ plane - the exponent of $\rho(\delta)$ serves as an order parameter describing the "phase transitions" to different anomalous scalings with different eddy diffusivity theories for the ensemble average. Such transitions are described by the "phase diagram" presented in Fig. 1. This phase diagram involves five regions with different anomalous scaling exponents with crossovers between diffusive, super-diffusive, and super-ballistic scaling regimes with corresponding remarkable changes in the nature of the effective equations for the eddy diffusivity theory. It is very interesting that the analogue of the Kolmogorov spectrum in the simple model occurs at the point $\tilde{\varepsilon}=8 / 3, z=2 / 3$ and these values correspond to a boundary point with "phase transition" between two different regions of renormalization (see Fig. 1 and page 409 of [1]). In other related work ([2,3]), the authors are using this simplified model with a rigorous exact renormalization theory to provide an unambiguous comparison of a variety of R-N-G methods and renormalized perturbation theories for predicting eddy diffusivity. In a completely different direction, the authors have recently used the predictions of the model to understand renormalization theory for general isotropic turbulent transportdiffusion in $R^{d}$ in the two phase regions analogous to those in Fig. 1 surrounding the Kolmogorov spectrum $([5,6])$.

This article has as main goals to pursue the study of model simple shear flows introduced in [1], focussing on three important topics: (i) Higher order statistics involving the relative diffusion of pairs of particles, described by the pair-distance distribution, and the closely related question of the evolution of interfaces in turbulent flows; (ii) the issue of statistical universality, i.e. exploring the dependence of the effective equations on the statistics of non-Gaussian random velocities; (iii) the "sweeping effect," arising from dispersion in a random flow with a uniform average velocity.

The phase-diagrams for the scaling laws and effective equations developed in [1] and in the present paper show remarkable crossovers in the neighborhood of the Kolmogorov-Obukhov regime $(\varepsilon=8 / 3, z=2 / 3)$. In this paper, we establish with complete rigor that the model flows reproduce several features associated with higher-order statistics of the passive scalar that have been observed (Lovejoy [27], Meneveau and Sreenivasan [31]), or are believed to occur in intermittency corrections to fully developed turbulence (Hentschel and Procaccia [17]). Our results include the evolution equation for the pair-distance distribution function, with explicit values for the corresponding relative diffusion coefficient, as well as calculation of the fractal dimensions of interfaces evolving in the flow. In particular, in the crossover between regions II and III, or II ${ }^{\bar{w}}$ and III ${ }^{\bar{w}}$ (see the phase diagrams below), we recover the analogous result for the model to Richardson's $l^{4 / 3}$ law [38] on one side of the boundaries II/III and $\mathrm{II}^{\bar{w}} / \mathrm{III}^{\bar{w}}$, and a different pair-dispersion 
diffusivity in regions III, III ${ }^{\bar{w}}$, proportional to $t l^{2 / 3}$, where $l$ denotes the interparticle separation. This last form of the pair diffusivity has been proposed on heuristic grounds in [17]. With regards to the fractal dimension of level sets, we show once again a remarkable crossover phenomenon in a neighborhood of the KolmogorovObukhov values. For values of the statistical parameters $\varepsilon=8 / 3$ and $z=2 / 3$, we obtain a dimensional defect $H=2-d=2 / 3=0.67$, and this value agrees also with the limit as the statistical parameters (defined below) $(\varepsilon, z)$ approach the Kolmogorov-Obukhov values from region II or II ${ }^{\bar{w}}$. Remarkably, the corresponding limit as $(\varepsilon, z)$ converge to the Kolmogorov-Obukhov values from inside regions III or III $^{\bar{w}}$ is $H=\frac{1}{3} \cong 0.33$, showing a discontinuity in the neighborhood of the homogeneous turbulence regime. Physically regions III and III $^{\bar{w}}$ correspond to regimes where macroscopically, time-decorrelation effects are negligible (Taylor's hypothesis). It is striking that the numerical values $H=\frac{2}{3}$ and $H=\frac{1}{3}$ are in very good agreement with recent high Reynolds number experiments [27], see also [12], and with values measured by Meneveau and Sreenivasan [31] in turbulent jets. Furthermore, with standard approximations from combustion theory involving turbulent diffusion flames [41], pp. 69-80), the evolution of such level sets coincides with the evolution of an interface defining the flame sheet. Thus, our results for the model give a rigorous estimate of the fractal dimension of interfaces such as diffusion flame fronts, evolving in the simple shear flows of the model. For these reasons, we often use the terminology, level set or interface interchangeably in Sect. 5.

To what extent are the renormalized eddy-diffusivity equations for passive transport by a random velocity field with a given power-energy spectrum dependent on the higher-order velocity statistics? This issue of statistical universality is very important from the view-point of turbulence modeling where explicit expressions for the sub-grid eddy-diffusivity are needed. In this paper, we show that the renormalization problem for random simple shear flows can be analyzed for a variety of non-Gaussian velocity statistics by means of appropriate central limit theorems, and that the corresponding renormalized equations agree with the ones for Gaussian flows. This universality of the eddy diffusivity for passive transport at high Reynolds numbers is shown again with full rigor for the models. The applicability of central limit theorems suggests that the results of this article also extend to isotropic 3D random flows. Some aspects of this extension for regions II and III are developed in [5] and [6] by the authors.

The study initiated in [1] is also extended to comprise velocities with a nonzero mean flow. This is also a simplified model problem for important practical issues, since most flows have a "mean wind." The consideration of a passively advected scalar in a coordinate system which moves with the mean flow leads to a Doppler-shifted velocity field with additional time-decorrelations. This "sweeping effect" arising from the mean velocity plays a role in the long-time/large-distance dynamics in some statistical regimes, but not in others. We show here how the phase-diagram for the models in the $(\varepsilon, z)$-plane, is modified by the presence of the mean "wind," and obtain a new phase-diagram which is useful to assess the sweeping effect in general random velocity fields.

As in our previous work [1], we consider transport by a simple shear flow, described by the equation

$$
\left(\frac{\partial}{\partial t}+\mathbf{u}_{\delta}(y, t) \cdot \nabla\right) T(x, y, t)=D\left[\frac{\partial^{2} T(x, y, t)}{\partial x^{2}}+\frac{\partial^{2} T(x, y, t)}{\partial y^{2}}\right]
$$


with initial condition

$$
T(x, y, t=0)=T_{0}(\delta x, \delta y)
$$

where $T_{0}(x, y)$ is an initial profile and $\delta$ is a small parameter representing the ratio $\eta / L$ between the dissipation and integral length scales [1]. (Here, we have changed the notation slightly from our earlier paper [1].) In (1.1) the velocity field $\mathbf{u}_{\delta}(y, t)$ is given by

$$
\mathbf{u}_{\delta}(y, t)=\left[\begin{array}{c}
\bar{v} \\
\bar{w}
\end{array}\right]+\left[\begin{array}{c}
u_{\delta}(y, t) \\
0
\end{array}\right],
$$

where $u_{\delta}(y, t)$ is a stationary random process and $\left[\begin{array}{c}\bar{v} \\ \bar{w}\end{array}\right]$ is a constant vector representing the average flow. We assume throughout this paper that the power-energy spectrum of $u_{\delta}(y, t)$ has the form

$$
\left\langle\hat{u}(k, \omega) \hat{u}\left(k^{\prime}, \omega^{\prime}\right)\right\rangle=E_{\delta}(k, \omega) \delta\left(k+k^{\prime}\right) \delta\left(\omega+\omega^{\prime}\right)
$$

with

$$
E_{\delta}(k, \omega)=\bar{U}^{2} \psi_{0}\left(\frac{k}{\delta}\right) \psi_{\infty}(k)|k|^{1-\varepsilon} \frac{1}{\pi\left[1+\omega^{2} / a^{2}|k|^{22}\right]} \cdot \frac{1}{a|k|^{2}} .
$$

In this formula, $\psi_{0}(k)$ and $\psi_{\infty}(k)$ are smooth, nonnegative, even functions (i.e., $\left.\psi_{i}(k)=\psi_{i}(-k), i=0, \infty\right)$, representing infrared and ultraviolet cutoffs. These functions are assumed to satisfy

$$
\psi_{0}(k)=\left\{\begin{array}{lll}
0 & \text { for } & |k| \leqq k_{0} \\
1 & \text { for } & |k| \geqq k_{1}
\end{array}\right.
$$

and

$$
\psi_{\infty}(k)=\left\{\begin{array}{lll}
1 & \text { for } & |k| \leqq k_{3} \\
0 & \text { for } & |k| \geqq k_{4}
\end{array} .\right.
$$

Such cutoffs are given naturally in turbulence theory by the integral scale and the dissipation scale respectively (see [1]). As in [1], the last condition in (1.7) can be replaced by rapid decay of ${ }_{+\infty} \psi_{\infty}(k)$ for $|k| \gg 1$. It follows that for $\delta k_{1} \leqq|k| \leqq k_{2}$, the energy spectrum $E_{\delta}(k)=\int_{-\infty} E_{\delta}(k, \omega) d \omega \propto \bar{U}^{2}|k|^{1-\varepsilon}$ is a power-law in $k$. The Fourier transform of $E_{\delta}(k, \omega)$ with respect to $\omega$, denoted by $E_{\delta}(k, t)$ satisfies

$$
\begin{aligned}
E_{\delta}(k, t) & =\left\langle\hat{u}_{\delta}(k, \tau+t) \overline{\hat{u}_{\delta}(k, \tau)}\right\rangle \\
& =\bar{U}^{2} \psi_{0}\left(\frac{k}{\delta}\right) \psi_{\infty}(k)|k|^{1-\varepsilon} e^{-\left.a|k|\right|_{t}}
\end{aligned}
$$

and indicates the rate of decorrelation in time of the random mode $\hat{u}(k, \tau)$. To understand the roles of the parameters $\varepsilon$ and $z$, we observe that, from (1.8), the exponent $\varepsilon$ controls the spatial correlations in the velocity field at fixed time, since

$$
E_{\delta}(k, 0)=\bar{U}^{2} \psi_{0}\left(\frac{k}{\delta}\right) \psi_{\infty}(k)|k|^{1-\varepsilon}
$$


so that, formally,

$$
\begin{aligned}
\left\langle u_{\delta}(x, t) u_{\delta}\left(x^{\prime}, t\right)\right\rangle & =\bar{U}^{2} \int \psi_{0}\left(\frac{k}{\delta}\right) \psi_{\infty}(k)|k|^{1-\varepsilon} e^{i k\left(x-x^{\prime}\right)} d k \\
& \sim \text { const. } \times\left|x-x^{\prime}\right|^{-2+\varepsilon} .
\end{aligned}
$$

In particular, large values of $\varepsilon$ correspond to long-range correlations in the velocity field. Concerning the exponent $z(z>0)$, we see from (1.8) that the decorrelation in time of $\hat{u}(k, t)$ for each $k$ is governed by the $k$-dependent turnover time

$$
\tau_{a}(k)=\frac{1}{a|k|^{2}} .
$$

This turnover time is longer for small values of $k$, thereby incorporating the feature that long-wavelength components oscillate more slowly than high- $k$ modes. In fact, in the limiting case $z=0$, all modes have identical turnover times, while $z>0$ corresponds to statistics for which modes with $|k| \ll 1$ have much larger turnover times than high modes. We have used the simple structural form of the function $\omega /|k|^{z}$ in (1.8) for simplicity in exposition; in fact, in regions II and II, any more general function $\Psi\left(\omega /|k|^{z}\right)$ can be used and yields the same renormalization theory, provided that $\Psi$ is integrable and nonzero in a neighborhood of the origin. A Doppler-shifted random function $\tilde{u}_{\delta}(y, t)=u_{\delta}(y+\bar{w} t, t)$, which corresponds to dispersion in a moving coordinate system, has a spectrum $\tilde{E}_{\delta}(k, \omega)$ given by

$$
\begin{aligned}
\tilde{E}_{\delta}(k, t) & =\bar{U}^{2} \psi_{0}\left(\frac{k}{\delta}\right) \psi_{\infty}(k)|k|^{1-\varepsilon} \cos (\bar{w} k t) e^{-a|k| z_{t}} \\
& =\cos (\bar{w} k t) E_{\delta}(k, t) .
\end{aligned}
$$

This gives rise to the $k$-dependent "sweeping" time

$$
\tau_{\bar{w}}(k)=\frac{1}{|k||\bar{w}|}
$$

which dominates the turnover time if $z>1$, i.e. $\tau_{\bar{w}}(k) \ll \tau_{a}(k)$ for $|k| \ll 1$, but is much larger than the turnover time if $z>1$.

The values of the parameters $\varepsilon$ and $z$ corresponding to homogeneous turbulence are, according to the Kolmogorov-Obukhov theory [22, 32],

$$
\varepsilon=\frac{8}{3}, \quad z=\frac{2}{3},
$$

and the corresponding spectrum is given by

$$
E_{\delta}(k, \omega)=\bar{U}^{2} \psi_{0}\left(\frac{k}{\delta}\right) \psi_{\infty}(k)|k|^{-5 / 3} \frac{\left(a|k|^{2 / 3}\right)^{-1}}{\pi\left(1+\omega^{2} / a^{2}|k|^{4 / 3}\right)} .
$$

By considering variable parameters $\varepsilon$ and $z$ we can study rigorously, in the context of these models, the renormalization problem for passive turbulent transport for Kolmogorov-Obukhov turbulence, as well as for regimes corresponding to intermittency corrections to the $k^{-5 / 3}$ law. Other statistical regimes corresponding to different $(\varepsilon, z)$ are interesting from the point of view of renormalization theory for 
flows in porous media $[10,11,15,21]$. Here, we consider the renormalization problem for all values of $\varepsilon, z$ in the ranges $-\infty<\varepsilon<4,0<z<\infty$.

We study the fundamental problem of finding a suitable scaling function $\rho=\rho(\delta)$ corresponding to the macroscopic evolution of the solution of the initial-value problem (1.1)-(1.2). This is done here by considering the scalar

$$
T_{\delta}\left(x^{\prime}, y^{\prime}, t^{\prime}\right)=T\left(\frac{x^{\prime}}{\delta}-\frac{\bar{v} t^{\prime}}{\rho(\delta)^{2}}, \frac{y^{\prime}}{\delta}-\frac{\bar{w} t^{\prime}}{\rho(\delta)^{2}}, \frac{t^{\prime}}{\rho(\delta)^{2}}\right)
$$

which satisfies

$$
\begin{aligned}
& \frac{\partial T_{\delta}\left(x^{\prime}, y^{\prime}, t^{\prime}\right)}{\partial t^{\prime}}+\frac{\delta}{\rho^{2}} u_{\delta}\left(\frac{y^{\prime}}{\delta}+\frac{\bar{w} t^{\prime}}{\rho(\delta)^{2}}, \frac{t^{\prime}}{\rho(\delta)^{2}}\right) \frac{\partial T_{\delta}\left(x^{\prime}, y^{\prime}, t^{\prime}\right)}{\partial x^{\prime}} \\
& =D\left[\frac{\partial^{2} T_{\delta}\left(x^{\prime}, y^{\prime}, t^{\prime}\right)}{\partial x^{\prime 2}}+\frac{\partial^{2} T_{\delta}\left(x^{\prime}, y^{\prime}, t^{\prime}\right)}{\partial y^{\prime 2}}\right]
\end{aligned}
$$

and

$$
T_{\delta}\left(x^{\prime}, y^{\prime}, 0\right)=T_{0}\left(x^{\prime}, y^{\prime}\right)
$$

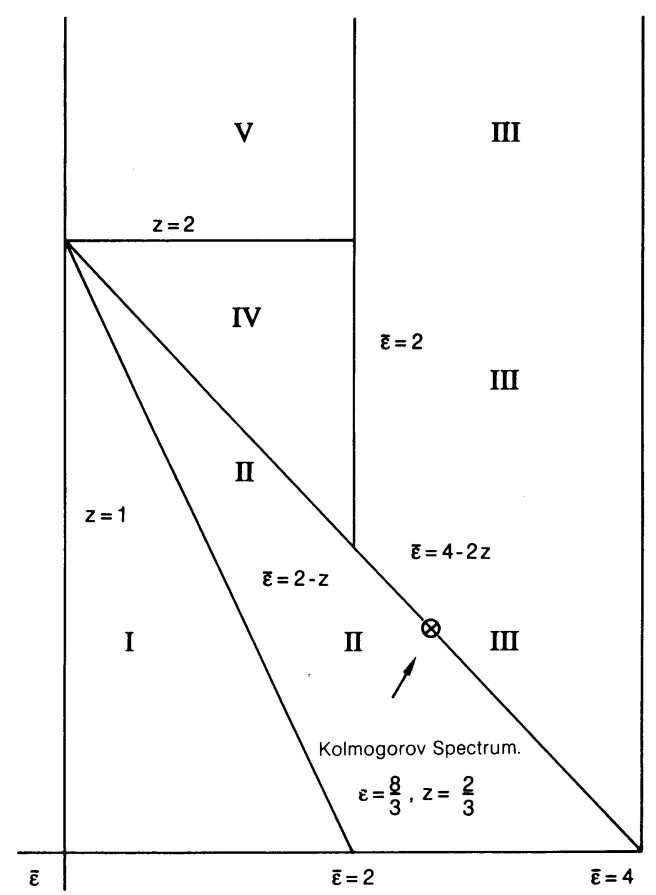

Fig. 1. The phase-diagram represents the five renormalization regimes for the case of vanishing transverse mean flow $(\bar{w}=0)$ according to the valves of the parameters $\varepsilon$ and $z$. The parameter $\varepsilon$ measures the strength of the infrared spectrum of the velocity, corresponding to long wavelength modes, while $z$ measures the rate of decorrelation in time of long wavelength modes (cf. (1.8), (1.9), (1.10)). The values $\varepsilon=8 / 3, z=2 / 3$ correspond to the Kolmogorov-Obukhov theory of homogeneous turbulence; they correspond to a point lying on the boundary between regions II and III of the phase-diagrams 


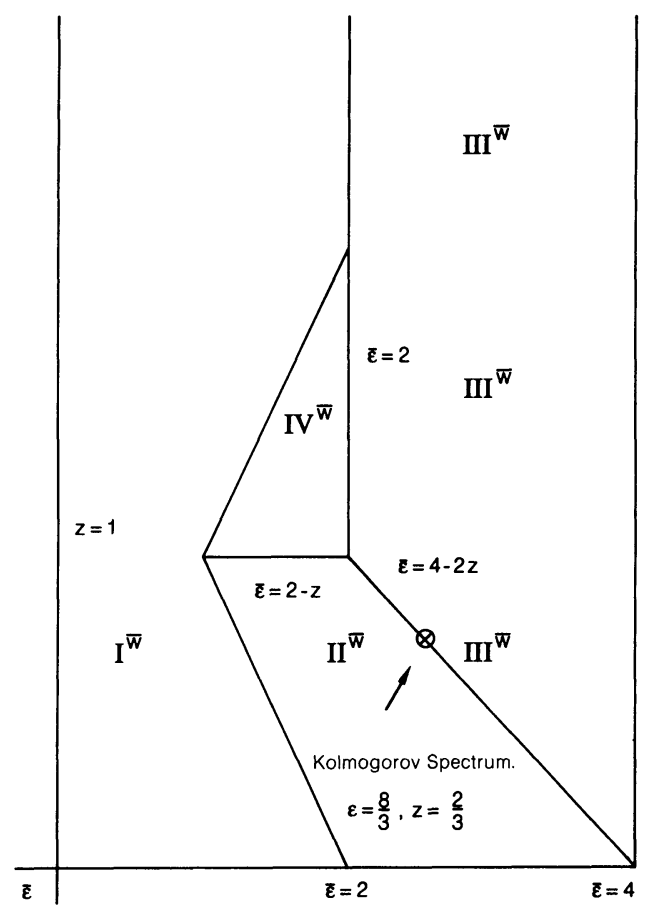

Fig. 2. The phase-diagram for $\bar{w} \neq 0$, incorporating the "sweeping effect" caused by a constant mean flow with non-vanishing transverse component $(\bar{w} \neq 0)$. Notice that the diagram has four different regions and differs from the one of Fig. 1 only for $z \geqq 1$. In particular, the diagram remains unchanged in the vicinity of the Kolmogorov-Obukhov values

Here, $x^{\prime}, y^{\prime}, t^{\prime}$ denote macroscopic space-time coordinates and an appropriate Galilean transformation to a frame evolving with the mean flow is made in (1.16), (1.17). The choice of the scaling function $\rho=\rho(\delta)$ must be such that the averaged scalar $\left\langle T_{\delta}\left(x^{\prime}, y^{\prime}, t^{\prime}\right)\right\rangle$ satisfies

$$
\lim _{\delta \downarrow 0}\left\langle T_{\delta}\left(x^{\prime}, y^{\prime}, t^{\prime}\right)\right\rangle=\bar{T}\left(x^{\prime}, y^{\prime}, t^{\prime}\right),
$$

where $\bar{T}\left(x^{\prime}, y^{\prime}, t^{\prime}\right)$ evolves according to an effective equation of motion. The correct scaling function $\rho(\delta)$ as well as the effective equation will depend, in general, on the statistical parameters $\varepsilon$ and $z$, as shown in [1] for the special case of Gaussian fields with $\bar{w}=0$. This deceivingly simple equation reveals a rich variety of different renormalization regimes as $\varepsilon, z$ vary. The regimes, or phases in the $(\varepsilon, z)$ plane, corresponding to different scaling functions and effective equations for the case $\bar{w}=0$, in (1.3), are shown in the phase-diagram of Fig. 1. These results are identical to those of [1] for Gaussian flows. In contrast, the phase-diagram for $\bar{w} \neq 0$, which is quite different, is shown in Fig. 2. In both cases, the Kolmogorov-Obukhov regime corresponds to a point on the boundary between two regions (II and III, if $\bar{w}=0$ or $\mathrm{II}^{\bar{w}}$ and III ${ }^{\bar{w}}$, if $\bar{w} \neq 0$ ). The consequences of this fact, which were outlined above, are studied in detail in Sects. IV and V hereafter. The differences between the diagrams 
for $\bar{w}=0$ and $\bar{w} \neq 0$ are significant only for $z>1$, which corresponds to the region in which the "sweeping time" $\tau_{\bar{w}}(k)$ is much shorter than the turnover time $\tau_{a}(k)$ for $|k| \ll 1$. In particular, the sweeping effect caused by $\bar{w} \neq 0$ is negligible for $\varepsilon, z$ in a neighborhood of the Kolmogorov regime. The chart in Table 1 describes the forms of the scaling functions $\rho(\delta)$ and the explicit form of the effective diffusivity of the effective equation of motion for $\bar{T}\left(x^{\prime}, y^{\prime}, t^{\prime}\right)$ (we drop the primes for simplicity). In regions $I$ and $I^{\bar{w}}$, which correspond to mean-field behavior, the effective equation is

$$
\frac{\partial \bar{T}(x, y, t)}{\partial t}=D^{*} \frac{\partial^{2} \bar{T}(x, y, t)}{\partial x^{2}}+D \frac{\partial^{2} T(x, y, t)}{\partial y^{2}}
$$

where $D^{*}$ is a renormalized effective diffusivity. Effective diffusivities for the remaining regions, which correspond to superdiffusive scalings with $\rho(\delta) \ll \delta$, II-V and $I I^{\bar{w}}-I^{\bar{w}}$ are given in Table 1 . Notice that the diffusivities can be local and time-independent (II, II ${ }^{\bar{w}}$, IV $^{\bar{w}}$ ), local and time-dependent (III, III ${ }^{\bar{w}}$, IV and boundaries II/III and $\mathrm{II}^{\bar{w}} / \mathrm{III}^{\bar{w}}$ ) or nonlocal (V). In the latter case, the nonlocal eddy diffusivity is the one studied in our previous paper [1]. Comparison of the diagrams for $\bar{w}=0$ and $\bar{w} \neq 0$ shows, among other things, that the presence of a mean-flow extends the region of validity of mean-field theory and suppresses the nonlocal superdiffusive region $V$.

Table 1. Summary of the scaling functions $\rho(\delta)$ and the effective diffusivities for all regions of both phase-diagrams, and for the boundaries II/III and II ${ }^{\bar{w}} / \mathrm{III}^{\bar{w}}$. Regions I and I ${ }^{\bar{w}}$ correspond to Fick's law of diffusion, so that $\rho(\delta)=\delta$. In all other regimes the motion is superdiffusive, with $\rho(\delta) \ll \delta$, as $\delta \rightarrow 0$

\begin{tabular}{|c|c|c|}
\hline Region & Scaling function & Effective diffusivity $\left(D^{*}\right)$ \\
\hline $\mathrm{I}, \mathrm{I}^{\bar{w}}$ & $\rho(\delta)=\delta$ & $\begin{array}{l}D+2 \bar{U}^{2} \\
\int_{-\infty}^{+\infty} \frac{\left[D|k|^{2}+a|k|^{2}\right]|k|^{1-\varepsilon} \psi_{\infty}|k| d k}{\left[D|k|^{2}+a|k|^{2}\right]^{2}+\bar{w}|k|^{2}}\end{array}$ \\
\hline $\mathrm{II}, \mathrm{II}^{\bar{w}}$ & $\rho(\delta)=\delta^{(4-\varepsilon-z) / 2}$ & $\frac{\bar{U}^{2}}{a} \int_{-\infty}^{+\infty} \psi_{0}(k)|k|^{1-\varepsilon-z} d k$ \\
\hline III, III ${ }^{\bar{w}}$ & $\rho(\delta)=\delta^{1-/ \varepsilon / 4}$ & $\frac{\bar{U}^{2}}{2} \int_{-\infty}^{+\infty} \psi_{0}(k)|k|^{1-\varepsilon} d k$ \\
\hline IV & $\rho(\delta)=\delta^{z /(\varepsilon+2 z-2)}$ & $\begin{array}{l}\left(2+\frac{\varepsilon-2}{z}\right) \frac{\bar{U}^{2}}{a}(a t)^{1+(\varepsilon-2) / z} \\
\cdot \int_{-\infty}^{+\infty}|k|^{1-\varepsilon-z}\left[1-\frac{1-e^{-|k|^{z}}}{|k|^{z}}\right] d k\end{array}$ \\
\hline $\mathrm{IV}^{\bar{w}}$ & $\rho(\delta)=\delta^{1-(\varepsilon-z) / 2}$ & $\frac{\bar{U}^{2} a}{\bar{w}^{2}} \int_{-\infty}^{+\infty} \psi_{0}(k)|k|^{1-\varepsilon+z} d k$ \\
\hline V & $\rho(\delta)=\delta^{1 /(1+\varepsilon / 2)}$ & Nonlocal (see [1] and Sect. III) \\
\hline Boundaries & $\rho(\delta)=\delta^{(4-\varepsilon-z) / 2}$ & $\frac{U^{2}}{a} \int_{-\infty}^{+\infty} \psi_{0}(k)|k|^{1-\varepsilon} e^{-a|k| z t} d k$ \\
\hline
\end{tabular}


Table 2. This table shows the explicit formulas for the pair-distance diffusivities for the models. With the exception of regions I, $\mathrm{I}^{\bar{w}}$, IV and V, the pair-diffusivities depend on the initial vertical separation of pairs of particles, and can be both time-dependent or time-independent, according to the region of interest. The Hölder exponent of the macroscopic front, $\bar{F}(y, t)$, as well as the fractal dimension of the front $d=2-H$ are given in each case

\begin{tabular}{|c|c|c|c|}
\hline Region & Pair-distance diffusivity $\left(D^{*}(l, t)\right)$ & $H$ & $d$ \\
\hline $\begin{array}{l}\mathrm{I}, \mathrm{I}^{\bar{w}}, \mathrm{IV} \\
\mathrm{V}\end{array}$ & $\begin{array}{l}2 \mathrm{D}^{*} \\
\text { nonlocal }\end{array}$ & $\begin{array}{l}0 \\
0\end{array}$ & $\begin{array}{l}2 \\
2\end{array}$ \\
\hline II, II ${ }^{\bar{w}}$ & $\frac{2 \bar{U}^{2}}{a} \int_{-\infty}^{+\infty}|k|^{1-\varepsilon-z}\left(1-\cos k l_{2}\right) \psi_{0}(k) d k$ & $\frac{\varepsilon+z-2}{2}$ & $\frac{6-\varepsilon-z}{2}$ \\
\hline III, III ${ }^{\bar{w}}$ & $\bar{U}^{2} t \int_{-\infty}^{+\infty}|k|^{1-\varepsilon}\left(1-\cos k l_{2}\right) \psi_{0}(k) d k$ & $\frac{\varepsilon-2}{2}$ & $\frac{6-\varepsilon}{2}$ \\
\hline$I V^{\bar{w}}$ & $\frac{2 \bar{U}^{2} a}{\bar{w}^{2}} \int_{-\infty}^{+\infty}|k|^{1-\varepsilon+z}\left(1-\cos k l_{2}\right) \psi_{0}(k) d k$ & $\frac{\varepsilon-z}{2}$ & $\frac{4+z-\varepsilon}{2}$ \\
\hline Boundaries & $\frac{2 \bar{U}^{2} a}{\bar{w}^{2}} \int_{-\infty}^{+\infty}|k|^{1-\varepsilon}\left(1-\cos k l_{2}\right) e^{-\left.a|k|\right|^{z} t} \psi_{0}(k) d k$ & $\frac{\varepsilon+z-2}{2}$ & $\frac{6-\varepsilon-z}{2}$ \\
\hline
\end{tabular}

Table 2 contains the results concerning the pair-distance diffusivity for relative diffusion, as well as the Hölder exponents and fractal dimensions of interfaces. The models reveal a rich structure, in which different pair-diffusivities emerge in each phase. In some regions $\left(\mathrm{I}, \mathrm{I}^{w}, \mathrm{IV}, \mathrm{V}\right)$, pairs of particles evolve independently in the macroscopic time-scale, and hence the pair-diffusivity for the pair-distance distribution is equal to twice the value of the (single-particle) effective diffusivity. On the other hand, the pair-diffusivity in regions II, II ${ }^{\bar{w}}$, III, III ${ }^{\bar{w}}, \mathrm{IV}^{\bar{w}}$, and the boundaries between II and III, and $\mathrm{II}^{\bar{w}}$ and III ${ }^{\bar{w}}$ depend on the initial vertical separation $l_{2}$ and on time in some cases (III; III ${ }^{\bar{w}}$ and boundaries) but is time-independent in others (II, $\mathrm{II}^{\bar{w}}$ ). Table 2 also indicates the fractal dimension of advected fronts, $d=2-H$. In regions I and $\mathrm{I}^{\bar{w}}$ the fronts are $\delta$-correlated on the macroscopic scales, and thus $d=2$. The same is true for regions IV and $\mathrm{V}$, with the difference that the fractal dimension is, in these cases scale-dependent, with an intermediate fractal dimension $1<d_{\text {int }}<2$ developing on time-scales which are large but yet smaller than the macroscopic time scale $\rho(\delta)^{-2}$. These results, which are not directly relevant to homogeneous turbulence will be described in a separate work. Regions II, II ${ }^{\bar{w}}$, III, $\mathrm{III}^{\bar{w}}, \mathrm{IV}^{\bar{w}}$ and the boundaries II/III, II ${ }^{\bar{w}} / \mathrm{III}^{\bar{w}}$ have fractal dimensions $d$ satisfying $1<d<2$, as shown in Table 2. An important conclusion that can be drawn from this analysis is that although scaling exponents vary continuously across phaseboundaries (in general), the quantities associated with higher order statistics (pair-distance distribution, fractal dimension of fronts) exhibit typically discontinuities across phase boundaries. We believe that this phenomenon may explain the consistent appearance of certain dimensional defects in experimental data [31]. [Note that for purposes of comparison the analogue of our line element in 3D models is a surface and the formula relating $H$ to $d$ should be $d=3-H$ instead of $d=2-H$.] 


\section{The Phase Diagram with the Sweeping Effect: Gaussian Statistics}

This section concerns the derivation of the different renormalization regimes for the advection-diffusion equation with random Gaussian velocity

$$
\begin{aligned}
& \frac{\partial T(x, y, t)}{\partial t}+\left(\bar{v}+u_{\delta}(y, t)\right) \frac{\partial T(x, y, t)}{\partial x}+\bar{w} \frac{\partial T(x, y, t)}{\partial y} \\
& =D\left(\frac{\partial^{2} T(x, y, t)}{\partial x^{2}}+\frac{\partial^{2} T(x, y, t)}{\partial y^{2}}\right)
\end{aligned}
$$

with initial data

$$
T(x, y, t=0)=T_{0}(\delta x, \delta y) .
$$

We shall focus primarily on the implications of having a nonzero transverse component of the mean flow, $\bar{w} \neq 0$. The case $\bar{w}=0$ was treated previously in [1]. For non-zero mean fields we consider Eq. (2.1) in a moving coordinate system, setting

$$
\begin{gathered}
x=x^{\prime}+t \bar{v}, \quad y=y^{\prime}+t \bar{w}, \\
T^{\prime}\left(x^{\prime}, y^{\prime}, t\right)=T\left(x^{\prime}+\bar{v} t, y^{\prime}+\bar{w} t, t\right) .
\end{gathered}
$$

The scalar $T^{\prime}$ satisfies the evolution equation

$$
\frac{\partial T^{\prime}\left(x^{\prime}, y^{\prime}, t\right)}{\partial t}+u_{\delta}\left(y^{\prime}+\bar{w} t, t\right) \frac{\partial T^{\prime}\left(x^{\prime}, y^{\prime}, t\right)}{\partial x^{\prime}}=D\left[\frac{\partial^{2} T^{\prime}\left(x^{\prime}, y^{\prime}, t\right)}{\partial x^{\prime 2}}+\frac{\partial^{2} T^{\prime}\left(x^{\prime}, y^{\prime}, t\right)}{\partial y^{\prime 2}}\right],
$$

with initial condition $T^{\prime}\left(x^{\prime}, y^{\prime}, 0\right)=T_{0}\left(\delta x^{\prime}, \delta y^{\prime}\right)$. As discussed in the Introduction, the renormalization problem consists, on the one hand, in determining a time-scaling function $\rho(\delta)$ such that the average scalar

$$
\left\langle T^{\prime}\left(\frac{x^{\prime}}{\delta}, \frac{y^{\prime}}{\delta}, \frac{t}{\rho(\delta)^{2}}\right)\right\rangle
$$

has a non-trivial limit as $\delta \rightarrow 0$, and, on the other hand, in characterizing the evolution of the limiting function

$$
\bar{T}\left(x^{\prime}, y^{\prime}, t\right)=\lim _{\delta \downarrow}\left\langle T^{\prime}\left(x^{\prime} / \delta, y^{\prime} / \delta, t / \rho(\delta)^{2}\right)\right\rangle .
$$

One of the main conclusions of our theory is that the scaling properties and effective evolution equations for $\bar{T}$ can vary substantially, according to the statistical correlations of the fluctuating field $u_{\delta}(y, t)$. To bring this into the foreground, we first study Gaussian velocity fields $u_{\delta}(y, t)$ with mean zero and power-energy spectrum

$$
E_{\delta}(k, \omega)=\bar{U}^{2} \psi_{0}^{2}\left(\frac{k}{\delta}\right) \psi_{\infty}^{2}(k)|k|^{1-\varepsilon}\left[\frac{1}{a|k|^{2}} \Phi\left(\frac{w}{a|k|^{2}}\right)\right]
$$

where

$$
\Phi(s)=\frac{1}{\pi} \frac{1}{1+s^{2}} .
$$


Accordingly, let $W(d k, d \omega)$ denote a two-dimensional, real white-noise Gaussian measure in $\mathbf{R}^{2}$, satisfying

$$
\begin{aligned}
& \iint_{A} W(d k, d \omega) \text { is Gaussian for all Borel sets } A \text { in } \mathbf{R}^{2}, \\
& \qquad\langle W(d k, d \omega)\rangle=0
\end{aligned}
$$

and

$$
\left\langle W(d k, d \omega) W\left(d k^{\prime}, d \omega^{\prime}\right)\right\rangle=\delta\left(k+k^{\prime}\right) \delta\left(\omega+\omega^{\prime}\right) d k d \omega .
$$

We define $u_{\delta}(y, t)$ (in this section) as

$$
u_{\delta}(y, t)=\bar{U} \iint e^{i k y+i \omega t} \psi_{0}^{1 / 2}\left(\frac{k}{\delta}\right) \psi_{\infty}^{1 / 2}(k)|k|^{1-\varepsilon / 2}\left[\frac{1}{a|k|^{z}} \Phi\left(\frac{\omega}{a|k|^{z}}\right)\right]^{1 / 2} W(d k, d \omega) \text {. }
$$

The long-time, large-distance problem for $(2.1-2.2)$ can then be studied as the statistical parameters $\varepsilon$ and $z$ are varied. Due to the presence of the shift $y \rightarrow y^{\prime}+\bar{w} t$, (i.e. the fact that $u_{\delta}\left(y^{\prime}+\bar{w} t, t\right)$ appears in (2.4)) the situation is different than in the case of a purely fluctuating velocity, previously studied in [1]. In the present case, the solution of the renormalization problem is summarized in the second phasediagram in the Introduction. What follows is a derivation of the scaling functions and effective evolution equations corresponding to each of the four nontrivial renormalization regimes in the diagram.

II.1. Normal Diffusion: Region $I^{\bar{w}}$. If the parameters $\varepsilon, z$ satisfy $z>0, \varepsilon+2 \min (1, z)-$ $\min (2, z)<2$, we shall see that the renormalization of $(2.1),(2.2)$ reduces to a problem in homogenization theory for operators with random coefficients, a question that was extensively studied by Papanicolaou and Varadhan [37] and others $[7,23,33,36]$. Choosing the time-scaling function $\rho(\delta)$ so that

$$
\rho(\delta)=\delta,
$$

which corresponds to Fick's law $\left(x^{2} \sim t\right)$, the equation satisfied by the scalar

$$
T_{\delta}\left(x^{\prime}, y^{\prime}, t\right)=T^{\prime}\left(\frac{x^{\prime}}{\delta}, \frac{y^{\prime}}{\delta}, \frac{t}{\delta^{2}}\right)
$$

is (dropping the primes):

$$
\left\{\begin{array}{l}
\frac{\partial T_{\delta}(x, y, t)}{\partial t}+\frac{1}{\delta} u_{\delta}\left(\frac{y}{\delta}+\bar{w} \frac{t}{\delta^{2}}, \frac{t}{\delta^{2}}\right) \frac{\partial T_{\delta}(x, y, t)}{\partial x} \\
=D\left[\frac{\partial^{2} T_{\delta}(x, y, t)}{\partial x^{2}}+\frac{\partial^{2} T_{\delta}(x, y, t)}{\partial y^{2}}\right] \\
T_{\delta}(x, y, 0)=T_{0}(x, y)
\end{array}\right.
$$

Without loss of generality, we consider plane-wave initial data of the form

$$
T_{0}(x, y)=e^{i x \cdot \xi+i y \cdot \eta}
$$

where $\xi, \eta$ are given numbers.

The solution of Eqs. (2.13), (2.14) can be represented probabilistically using a two-dimensional Brownian motion $\left(\beta_{1}(t), \beta_{2}(t)\right)$, (cf. for instance, McKean [30]). 
Accordingly,

$$
\begin{aligned}
& T_{\delta}(x, y, t) \\
& =e^{i x \cdot \xi+i y \cdot \eta} \times E\left\{\operatorname { e x p } \left[i \xi \sqrt{2 D} \beta_{1}(t)+i \eta \sqrt{2 D} \beta_{2}(t)\right.\right. \\
& \left.\left.\quad+\frac{i \xi}{\delta} \int_{0}^{t} u_{\delta}\left(\frac{y}{\delta}+\frac{\sqrt{2 D}}{\delta} \beta_{2}(s)+\frac{\bar{w}_{s}}{\delta^{2}}, \frac{s}{\delta^{2}}\right) d s\right]\right\} \\
& =e^{i \xi x+i \eta y} e^{-\xi^{2} D t} E\left\{\exp \left[i \eta \sqrt{2 D} \beta_{2}(t)+i \frac{\xi}{\delta} \int_{0}^{t} u_{\delta}\left(\frac{y}{\delta}+\frac{\sqrt{2 D}}{\delta} \beta_{2}(s)+\frac{\bar{w} s}{\delta^{2}}, \frac{s}{\delta^{2}}\right) d s\right]\right\},
\end{aligned}
$$

where $E\{\cdot\}$ denotes averaging over $\beta_{l}(t), \beta_{2}(t)$. Following Bensoussan-LionsPapanicolaou [7] and Papanicolaou-Varadhan [35], we can show that for $\varepsilon, z$ in region $\mathrm{I}^{\bar{w}}$, the exponent in (2.15),

$$
\eta \sqrt{2 D} \beta_{2}(t)+\frac{\xi}{\delta} \int_{0}^{t} u_{\delta}\left(\frac{y+\sqrt{2 D} \beta_{2}(s)}{\delta}+\frac{\bar{w} s}{\delta^{2}}, \frac{s}{\delta^{2}}\right) d s,
$$

converges in distribution to a Gaussian random variable with mean zero and variance

$$
\left[2 D \eta^{2}+2\left(D^{*}-D\right) \xi^{2}\right] t,
$$

where $D^{*}$ is the longitudinal diffusivity, given by

$$
D^{*}=D+2 \bar{U}^{2} \int_{-\infty}^{+\infty} \frac{\left(D|k|^{2}+a|k|^{2}\right)|k|^{1-\varepsilon} \psi_{\infty}(k) d k}{\left[D k^{2}+a|k|^{2}\right]^{2}+\bar{w}^{2} k^{2}} .
$$

Using this result, and the representation formula (2.15), we conclude that

$$
\begin{aligned}
\bar{T}(x, y, t) & =\lim _{\delta \downarrow 0}\left\langle T_{\delta}(x, y, t)\right\rangle \\
& =e^{i \xi x+i \eta y} e^{-\xi^{2} D t} e^{-\left(\eta^{2} D+\left(D^{*}-D\right) \xi^{2}\right) t} \\
& =e^{i \xi x+i \eta y} e^{-\left(D^{*} \xi^{2} t+D \eta^{2} t\right)} .
\end{aligned}
$$

The proof of the convergence in distribution of the random variable (2.16) to a Gaussian random variable with mean zero and variance (2.17) is given in Appendix 1. Notice that the condition $\varepsilon+2 \min (1, z)-\min (2, z)<2$ is necessary and sufficient for the convergence of the integral in (2.18) defining the effective longitudinal diffusivity $D^{*}$. Since Eq. (2.13) is linear, using (2.19), we conclude that, for general smooth initial data $T_{0}(x, y)$, the function $\bar{T}(x, y, t)$ defined by (2.6) evolves according to the effective diffusion equation

$$
\left\{\begin{array}{l}
\frac{\partial \bar{T}(x, y, t)}{\partial t}=D^{*} \frac{\partial^{2} \bar{T}(x, y, t)}{\partial x^{2}}+D \frac{\partial^{2} \bar{T}(x, y, t)}{\partial y^{2}} \\
\bar{T}(x, y, 0)=T_{0}(x, y)
\end{array}\right.
$$

II.2. Anomalous Diffusion. For $\varepsilon, z$ outside the mean-field region $I^{\bar{w}}$, the behavior of (2.1), (2.2) is superdiffusive, in the sense that $\rho(\delta) \ll \delta$ as $\delta \rightarrow 0$. There are altogether 
three different regions in the $(\varepsilon, z)$ plane in which superdiffusion occurs. In each region, the macroscopic behavior is determined by a different balance between spatial and temporal correlations of the fluctuating velocity. To put this in evidence, we use the probabilistic representation for the solution of the initial value problem (2.4) in terms of a Brownian path-space integral. The determination of the different renormalization regimes is done by studying the scaling properties of this path space integral in the long-time/large-distance limit as $\varepsilon$ and $z$ vary.

We begin by giving a suitable representation formula for $\left\langle T^{\prime}\left(x^{\prime}, y^{\prime}, t\right)\right\rangle$, where $T^{\prime}$ is defined in (2.4). Dropping the primes for simplicity, we have (cf. [30])

$$
T^{\prime}(x, y, t)=E\left\{T_{0}(\delta X(t), \delta Y(t))\right\},
$$

where $X(t), Y(t)$ are the coordinates of a Lagrangian particle in a reference frame moving with the mean flow, i.e.,

$$
\begin{aligned}
& X(t)=x+\sqrt{2 D} \beta_{1}(t)+\int_{0}^{t} u_{\delta}\left(y+\sqrt{2 D} \beta_{2}(s)+\bar{w} s, s\right) d s \\
& Y(t)=y+\sqrt{2 D} \beta_{2}(t)
\end{aligned}
$$

For plane-wave initial data $T_{0}$, of the form (2.14), substitution of (2.22) in (2.21) yields

$$
\begin{aligned}
& T^{\prime}(x, y, t) \\
& =e^{i \delta \xi \cdot x+i \delta \eta \cdot y} E\left\{\operatorname { e x p } \left[i \delta \xi \sqrt{2 D} \beta_{1}(t)+i \delta \xi \int_{0}^{t} u_{s}\left(y+\sqrt{2 D} \beta_{2}(s)+\bar{w} s, s\right) d s\right.\right. \\
& \left.\left.+i \delta \eta \sqrt{2 D} \beta_{2}(t)\right]\right\} \\
& =e^{i \delta \xi \cdot x+i \delta \eta \cdot y-\delta^{2} \xi^{2} D t} E\left\{\exp \left[i \delta \eta \sqrt{2 D} \beta_{2}(t)+i \delta \xi \int_{0}^{t} u_{\delta}\left(y+\sqrt{2 D} \beta_{2}(s)+\bar{w} s, s\right) d s\right]\right\} \text {. }
\end{aligned}
$$

Using the fact that $u_{\delta}$ is Gaussian, we can compute explicitly the average of $T^{\prime}(x, y, t)$ with respect to velocity statistics. We obtain, accordingly,

$$
\begin{aligned}
&\left\langle T^{\prime}(x, y, t)\right\rangle \\
&= e^{i \delta \xi x+i \delta \eta y-\delta^{2} \xi^{2} D t} \\
& \cdot E\left\{\exp \left[i \delta \eta \sqrt{2 D} \beta_{2}(t)-\frac{\delta^{2} \xi^{2}}{2}\left\langle\left[\int_{0}^{t} U_{\delta}\left(y+\sqrt{2 D} \beta_{2}(s)+\bar{w} s, s\right) d s\right]^{2}\right\rangle\right]\right\} \\
&= e^{i \delta \xi \cdot x+i \delta \eta y-\delta^{2} \xi^{2} D t} \\
& \cdot E\left\{\exp \left[i \eta \delta \sqrt{2 D} \beta_{2}(t)-\frac{\delta^{2} \xi^{2}}{2} \int_{0}^{t} \int_{0}^{t} R\left(\sqrt{2 D}\left(\beta(s)-\beta\left(s^{\prime}\right)\right)+\bar{w}\left(s-s^{\prime}\right), s-s^{\prime}\right) d s d s^{\prime}\right]\right\} .
\end{aligned}
$$

Here $R(y, t)$ is the Eulerian velocity autocorrelation function, given by

$$
\begin{aligned}
R(y, t) & =\iint e^{i k \cdot y+i w t} E_{\delta}(k, \omega) d k d \omega \\
& =\bar{U}^{2} \int_{-\infty}^{+\infty} \psi_{0}^{2}\left(\frac{k}{\delta}\right) \psi_{\infty}^{2}(k)|k|^{1-\varepsilon} e^{i k \cdot y-a|k| z t} d k .
\end{aligned}
$$


For notational simplicity, we set

$$
Q_{\delta}(t)=\int_{0}^{t} \int_{0}^{t} R\left(\sqrt{2 D}\left(\beta_{2}(s)-\beta_{s}\left(s^{\prime}\right)\right)+\bar{w}\left(s-s^{\prime}\right), s-s^{\prime}\right) d s^{\prime} d s
$$

After making the change of variables $x \rightarrow \frac{x}{\delta}, y \rightarrow \frac{y}{\delta}, t \rightarrow \frac{t}{\rho^{2}}$, where $\rho=\rho(\delta)$ is a yet unspecified function of $\delta$, we obtain

$$
\left\langle T^{\prime}\left(\frac{x}{\delta}, \frac{y}{\delta}, \frac{t}{\rho^{2}}\right)\right\rangle=e^{i \xi \cdot x+i \eta y-\left(\delta^{2} / \rho^{2}\right) D \xi^{2} t} E\left\{\exp \left[i \delta \eta \sqrt{2 D} \beta_{2}\left(\frac{t}{\rho^{2}}\right)-\frac{\delta^{2} \xi^{2}}{2} Q_{\delta}\left(\frac{t}{\rho^{2}}\right)\right]\right\} .
$$

This is the basic representation formula that will be used to compute the timescaling functions and the effective equations of motion in the different anomalous renormalization regions. Outside region $\mathrm{I}^{\bar{w}}$, we anticipate that the system should be superdiffusive, i.e. $\rho(\delta) \ll \delta$. Therefore, since we have $\beta_{2}\left(t / \rho^{2}\right)=\frac{1}{\rho} \tilde{\beta}_{2}(t)$, where $\tilde{\beta}_{2}(t)=\rho \beta_{2}\left(\frac{t}{\rho^{2}}\right)$ is another Brownian motion, the contributions from $\bar{e}^{\delta^{2} / \rho^{2} D \xi^{2} t}$ and $e^{i \delta \sqrt{2 D} \eta \beta_{2}\left(t / \rho^{2}\right)}$ in (2.27) should negligible as $\delta \rightarrow 0$, and the leading contribution should come from the exponential

$$
e^{-\left(\xi^{2} \delta^{2} / 2\right) Q \delta\left(t / \rho^{2}\right)}
$$

The differences in the asymptotic behavior of $Q_{\delta}\left(t / \rho^{2}\right)$ as $\rho \rightarrow 0$ determine the various "phases" of the diagram for the effective equations of motion, as shown in the following paragraphs.

\section{II.2.A. Superdiffusion with Rapid Time-Decorrelation: Region $I I^{\bar{w}}$}

Region II $^{\bar{w}}$ is determined by the inequalities

$$
0<z<1, \quad 2-z<\varepsilon<4-2 z \text {. }
$$

We make the choice of scaling function

$$
\rho(\delta)=\delta^{(4-\varepsilon-z) / 2}
$$

which, on account of (2.29), satisfies $\delta / \rho(\delta) \rightarrow 0$ as $\delta \rightarrow 0$. From (2.26), we have

$$
\begin{aligned}
& Q_{\delta}\left(\frac{t}{\rho^{2}}\right)=\bar{U}^{2} \int_{-\infty}^{+\infty} \psi_{0}\left(\frac{k}{\delta}\right) \psi_{\infty}(k)|k|^{1-\varepsilon} d k \\
& \quad \cdot\left[\int_{0}^{t / \rho^{2}} \int_{0}^{t / \rho^{2}} \exp \left[i k \sqrt{2 D}\left(\beta_{2}(s)-\beta_{2}\left(s^{\prime}\right)\right)+i \bar{w} k\left(s-s^{\prime}\right)-a|k|^{z}\left|s-s^{\prime}\right| d s d s^{\prime}\right]\right] .
\end{aligned}
$$

The quantity in brackets in this equation can be written, after a change of variables, 
in the form

$$
\frac{t^{2}}{\rho^{4}} \int_{0}^{1} \int_{0}^{1} \exp \left[i k \sqrt{2 D} \frac{t^{1 / 2}}{\rho}\left(\tilde{\beta}(s)-\tilde{\beta}\left(s^{\prime}\right)\right)+i k \bar{w} \frac{t}{\rho^{2}}\left(s-s^{\prime}\right)-a|k|^{z} \frac{t}{\rho^{2}}\left|s-s^{\prime}\right|\right] d s d s^{\prime},
$$

where $\tilde{\beta}(s)=\frac{\rho}{t^{1 / 2}} \beta_{2}\left(\frac{t}{\rho^{2}} s\right)$ is an auxiliary Brownian motion. If we make the substitution $k=\delta k^{\prime}$ in (2.32), this integral becomes

$\frac{t^{2}}{\rho^{4}} \int_{0}^{1} \int_{0}^{1} \exp \left[i k^{\prime} \sqrt{2 D} \frac{t^{1 / 2}}{\rho}\left(\tilde{\beta}(s)-\tilde{\beta}\left(s^{\prime}\right)\right)+i k^{\prime} \bar{w} \frac{\delta t}{\rho^{2}}\left(s-s^{\prime}\right)-a|k|^{z} \frac{\delta^{z} t}{\rho^{2}}\left|s-s^{\prime}\right|\right] d s d s^{\prime}$.

Since $\frac{\delta}{\rho} \ll 1$, and, for any $0<\alpha<1$, we have

$$
\exp \left[i k^{\prime} \frac{\delta t^{1 / 2}}{\rho} \sqrt{2 D}\left(\tilde{\beta}(s)-\tilde{\beta}\left(s^{\prime}\right)\right)\right]=1+0\left(\left(\frac{\delta k^{\prime}}{\rho}\right)^{\alpha}\right),
$$

the integral in (2.33), is asymptotically equivalent to

$$
\begin{gathered}
\frac{t^{2}}{\rho^{4}} \int_{0}^{1} \int_{0}^{1} \exp \left[i k^{\prime} \bar{w} \frac{\delta t}{\rho^{2}}\left(s-s^{\prime}\right)-a\left|k^{\prime}\right|^{z} \frac{\delta^{z} t}{\rho^{2}}\left|s-s^{\prime}\right|\right] d s d s^{\prime} \\
=\frac{2 t^{2}}{\rho^{4}} \int_{0}^{1} \int_{0}^{s} \exp \left[i k^{\prime} \bar{w} \frac{\delta t}{\rho^{2}} s^{\prime}-a|k|^{z} \frac{\delta^{z} t}{\rho^{2}} s^{\prime}\right] d s^{\prime} d s \\
=\frac{2 t^{2}}{\rho^{4}} \frac{1}{A\left(r^{\prime}, t, \delta\right)}\left[1-\frac{1-e^{-A\left(k^{\prime}, t, \delta\right)}}{A\left(k^{\prime}, t, \delta\right)}\right],
\end{gathered}
$$

with

$$
A\left(k^{\prime}, t, \delta\right)=a\left|k^{\prime}\right|^{z} \frac{\delta^{z} t}{\rho^{2}}-i k^{\prime} \bar{w} \frac{\delta t}{\rho^{2}} .
$$

From the definition of $\rho=\rho(\delta)$ in (2.30), we have

$$
\frac{\delta^{z}}{\rho^{2}}=\delta^{(\varepsilon+2 z-4) / 2} \rightarrow \infty \quad \text { as } \delta \rightarrow 0
$$

so that,

$$
\begin{aligned}
& \frac{t^{2}}{\rho^{4}} \int_{0}^{1} \int_{0}^{1} \exp \left[i k^{\prime} \frac{\delta}{\rho} \sqrt{2 D t}\left(\tilde{\beta}(s)-\tilde{\beta}\left(s^{\prime}\right)\right)+i k^{\prime} \frac{\bar{w} \delta}{\rho^{2}}\left(s-s^{\prime}\right)-a\left|k^{\prime}\right|^{z} \frac{\delta^{z}}{\rho^{2}} t\left|s-s^{\prime}\right|\right] d s d s^{\prime} \\
& \quad=\frac{2 t^{2}}{\rho^{4}} \frac{1}{\left(\frac{a\left|k^{\prime}\right|^{z} \delta^{z} t}{\rho^{2}}-i \frac{k^{\prime} \cdot \bar{w} \delta t}{\rho^{2}}\right)}(1+o(1)) \\
& =\frac{2 t}{\rho^{2} \delta^{z} a\left|k^{\prime}\right|^{z}}(1+o(1)) .
\end{aligned}
$$

Substituting this asymptotic expression in the integral in (2.31), (and recalling (2.34)) 
we obtain

$$
\frac{\xi^{2} \delta^{2}}{2} Q_{\delta}\left(\frac{t}{\rho^{2}}\right)=\frac{\xi^{2} t \delta^{4-\varepsilon} \bar{U}^{2}}{a \rho^{2} \delta^{z}}\left[\int \psi_{0}(k) \psi_{\infty}(\delta k)|k|^{1-\varepsilon-z} d k+o(1)\right] .
$$

Therefore, since $\rho(\delta)=\delta^{(4-\varepsilon-z) / 2}$, we have

$$
\frac{\xi^{2} \delta^{2}}{2} Q_{\delta}\left(\frac{t}{\rho^{2}}\right)=\frac{\xi^{2} t \bar{U}^{2}}{a} \int \psi_{0}(k)|k|^{1-\varepsilon-z} d k+o(1)
$$

and hence, from the representation formula (2.27),

$$
\lim _{\delta \downarrow 0}\left\langle T^{\prime}\left(\frac{x}{\delta}, \frac{y}{\delta^{\prime}}, \frac{t}{\rho^{2}}\right)\right\rangle=e^{i \xi x+i \eta y-\xi^{2} D_{\mathrm{II}}^{*} t}
$$

with

$$
D_{\mathrm{II}}^{*}=\frac{\bar{U}^{2}}{a} \int_{-\infty}^{+\infty} \psi_{0}(k)|k|^{1-\varepsilon-z} d k
$$

Clearly, our calculation for plane-wave initial data implies, more generally, that for arbitrary smooth initial data $T_{0}(x, y)$, the function $\bar{T}(x, y, t)=\lim _{\delta \downarrow 0}\left\langle T^{\prime}\left(\frac{x}{\delta}, \frac{y}{\delta}, \frac{t}{\rho^{2}}\right)\right\rangle$
satisfies the evolution equation

$$
\left\{\begin{array}{l}
\frac{\partial \bar{T}(x, y, t)}{\partial t}=D_{\mathrm{II}}^{*} \frac{\partial^{2} \bar{T}(x, y, t)}{\partial x^{2}} \\
\bar{T}(x, y, 0)=T_{0}(x, y)
\end{array}\right.
$$

This concludes the analysis of region $\mathrm{II}^{\bar{w}}$.

II.2.B. Superballistic Diffusion Dominated by Long-Range Spatial Correlations: Regions $I I I^{\bar{w}}$. Region III $^{\bar{w}}$ is determined by the inequalities

$$
\max (2,4-2 z)<\varepsilon<4 \text {. }
$$

The appropriate scaling function for this range of parameters $\varepsilon$ and $z$ is

$$
\rho(\delta)=\delta^{1-\varepsilon / 4} .
$$

With this value of $\rho(\delta)$, we have

$$
\left\{\begin{array}{l}
\frac{\delta^{z}}{\rho^{2}}=\delta^{(\varepsilon+2 z-4) / 2} \rightarrow 0 \\
\frac{\delta}{\rho^{2}}=\delta^{(\varepsilon-2) / 2} \rightarrow 0 \\
\frac{\delta}{\rho}=\delta^{\varepsilon / 4} \rightarrow 0
\end{array}\right.
$$

as $\delta \rightarrow 0$. Therefore, if we make the change of variables $k=\delta k^{\prime}$ in (2.31) we obtain

$$
Q_{\delta}\left(\frac{t}{\rho^{2}}\right)=\frac{\delta^{2-\varepsilon} t^{2} \bar{U}^{2}}{\rho^{4}} \int_{-\infty}^{+\infty} \psi_{0}\left(k^{\prime}\right) \psi_{\infty}\left(\delta k^{\prime}\right)\left|k^{\prime}\right|^{1-\varepsilon}\left[\int_{0}^{1} \int_{0}^{1} e^{-B\left(k^{\prime}, s, s^{\prime}, \delta\right)} d s d s^{\prime}\right] d k
$$


with

$$
B\left(k^{\prime}, s, s^{\prime}, \delta\right)=a\left|k^{\prime}\right|^{z} \frac{\delta^{z} t^{z}}{\rho^{2}}\left|s-s^{\prime}\right|+i \bar{w} k^{\prime} \frac{\delta t}{\rho^{2}}\left(s-s^{\prime}\right)+i k^{\prime} \frac{\delta}{\rho} \sqrt{2 D t}\left(\tilde{\beta}(s)-\widetilde{\beta}\left(s^{\prime}\right)\right),
$$

where $\tilde{\beta}(s)$ is an auxiliary Brownian motion. Using (2.46), we conclude that $B\left(k^{\prime}, s, s^{\prime}, \delta\right) \rightarrow 0$ as $\delta \rightarrow 0$. Hence

$$
\frac{\delta^{2} \xi^{2}}{2} Q_{\delta}\left(\frac{t}{\rho^{2}}\right)=\left(\frac{\delta^{4-\varepsilon}}{\rho^{4}}\right)\left[\frac{\xi^{2} t^{2}}{2} \bar{U}^{2} \int_{-\infty}^{+\infty} \psi_{0}\left(k^{\prime}\right)\left|k^{\prime}\right|^{1-\varepsilon} d k^{\prime}+o(1)\right]
$$

The choice $\rho(\delta)=\delta^{1-\varepsilon / 4}$ in region III implies that the right-hand side of (2.49) is finite, so that the averaged scalar $\left\langle T^{\prime}\left(\frac{x}{\delta}, \frac{y}{\delta}, \frac{t}{\rho^{2}}\right)\right\rangle$, corresponding to a plane-wave initial data, satisfies, from (2.27),

$$
\begin{aligned}
\bar{T}(x, y, t) & =\lim _{\delta \downarrow 0}\left\langle T^{\prime}\left(\frac{x}{\delta}, \frac{y}{\delta}, \frac{t}{\rho^{2}}\right)\right\rangle \\
& =\exp \left[i \xi x+i \eta y-\frac{\xi^{2} t^{2} \bar{U}^{2}}{2} \int_{-\infty}^{+\infty} \psi_{0}(k)|k|^{1-\varepsilon} d k\right] .
\end{aligned}
$$

Therefore, defining the effective diffusivity

$$
D_{\mathrm{III}}^{*}(t)=\frac{\bar{U}^{2} t}{2} \int_{-\infty}^{+\infty} \psi_{0}(k)|k|^{1-\varepsilon} d k
$$

we conclude, from (2.50), that the evolution equation for the function $\bar{T}(x, y, t)$ for arbitrary smooth initial data $T_{0}(x, y)$, is

$$
\left\{\begin{array}{l}
\frac{\partial \bar{T}(x, y, t)}{\partial t}=D_{\mathrm{III}}^{*}(t) \frac{\partial^{2} \bar{T}(x, y, t)}{\partial x^{2}} \\
\bar{T}(x, y, 0)=T_{0}(x, y)
\end{array}\right.
$$

Notice that the function $\rho(\delta)=\delta^{1-\varepsilon / 4}$ corresponds to the scaling relation

$$
X^{2} \sim T^{1 /(1-\varepsilon / 4)}
$$

for the displacement of advected Lagrangian particles on length scales on the order of $\delta^{-1}$. In particular, since $\varepsilon>2$ in region III $^{\bar{w}},(1-\varepsilon / 4)^{-1}>2$ and hence $X^{2} \gg T^{2}$. This apparently paradoxical, "superballistic" motion is a consequence of the fact that the kinetic energy $\left\langle\left|u_{\delta}(0,0)\right|^{2}\right\rangle$ diverges in region III ${ }^{\bar{w}}$ as $\delta \rightarrow 0$. Physically, this means that in region III $^{\bar{w}}$ the motion over large scales is influenced by an increasingly large input of kinetic energy in the long-wavelength modes. This results in an apparent average particle velocity $X / T$ that diverges with the size of the system. Notice that superballistic motion also exists in a portion of region $\mathrm{II}^{\bar{w}}$, for $3-z<\varepsilon<4-2 z$, and in particular on the boundary between II $^{\bar{w}}$ and III ${ }^{\bar{w}}$.

II.2.C. Superdiffusion with Rapid Time Decorrelation Arising from the Transverse Mean Flow: Region $I V^{\bar{w}}$. Region $I V^{\bar{w}}$ of the phase-diagram corresponds to values of $\varepsilon$ and $z$ for which the principal time-decorrelation effect is due to the "sweeping" of particles across the stratification by the mean velocity. This "sweeping effect" is felt at values of $z$ such that $z>1$, since, in such regime, the $k$-dependent "sweeping 
time" $\tau_{\bar{w}}(k)=\frac{1}{|\bar{w}||k|}$ is much smaller than the eddy turnover time $\tau_{a}(k)=\frac{1}{a|k|^{2}}$, for $|k| \ll 1$. This region is characterized by the inequalities

$$
1<z<\varepsilon<2 \text {. }
$$

As we shall demonstrate, appropriate the time-scaling function $\rho(\delta)$ in region $\mathrm{IV}^{\bar{w}}$ is given by

$$
\rho(\delta)=\delta^{1-(\varepsilon-z) / 2} .
$$

In order to justify (2.55) and compute the effective equation of motion, we study the behavior of the quantity $\frac{\xi^{2} \delta^{2} Q_{\delta}\left(t / \rho^{2}\right)}{2}$ as $\delta \rightarrow 0$, as we did in II.2.B. Note that, from the choice of $\rho(\delta)$ in (2.55), we have $\delta / \rho(\delta)=\delta^{(\varepsilon-z) / 2} \rightarrow 0$. It follows, from (2.31), (2.34), (2.35), and (2.36), that $\frac{\xi^{2} \delta^{2}}{2} Q_{\delta}\left(t / \rho^{2}\right)$ behaves, to leading order, as

$$
\frac{\xi^{2} \delta^{2}}{2} Q_{\delta}\left(\frac{t}{\rho^{2}}\right) \cong \bar{U}^{2} \frac{\xi^{2} \delta^{4-\varepsilon} t}{2 \rho^{2}} \int \frac{\psi_{0}(k) \psi_{\infty}(\delta k)|k|^{1-\varepsilon}}{\left(a|k|^{2} \delta^{z}-i k \bar{w} \delta\right)}\left[1-\frac{1-e^{-A(k, t, \delta)}}{A(k, t, \delta)}\right] d k,
$$

with $A(k, \delta, t)=\left(a|k|^{z} \delta^{z}-i \bar{w} k \delta\right) \frac{t}{\rho^{2}}$. After some computation, we find that the right-hand side of this equation is equal to

$$
\begin{aligned}
& \bar{U}^{2} \xi^{2} t \times \int \frac{\psi_{0}(k) \psi_{\infty}(\delta k)|k|^{1-\varepsilon+z} a}{a^{2}|k|^{2 z} \delta^{2(z-1)}+\bar{w}^{2}|k|^{2}}(1-B(k, t, \delta)) k \\
& +\bar{U}^{2} \xi^{2} t \times \delta^{2-\varepsilon}\left[\int \psi_{0}(k)|k|^{-(1+\varepsilon)} d k+o(1)\right] \\
& +\bar{U}^{2} \xi^{2} t \times \delta^{1+z-\varepsilon}\left[\int \psi_{0}(k)|k|^{-2-\varepsilon+z} a d k+o(1)\right],
\end{aligned}
$$

where $B(k, t, \delta)$ is given by

$$
\begin{aligned}
B(k, \delta, t)= & \frac{\rho^{4}}{\left(a^{2}|k|^{2 z} \delta^{2 z}+\bar{w}^{2} k^{2} \delta^{2}\right)} \\
& \cdot\left[\left(1-e^{-a\left(\left(\left.|k|\right|^{z} \delta^{z} t\right) / \rho^{2}\right)} \cos \left(\frac{\bar{w} k \delta t}{\rho^{2}}\right)\right)\left(\frac{a|k|^{2} \delta^{z} t}{\rho^{2}}\right)\right. \\
& \left.+\sin \left(\frac{\bar{w} k \delta t}{\rho^{2}}\right) e^{-a\left(\left(\left.|k|\right|^{2} \delta t\right) / \rho^{2}\right)} \cdot\left(\frac{\bar{w} k \delta t}{\rho^{2}}\right)\right]=0\left(\delta^{1-\varepsilon+z}\right) .
\end{aligned}
$$

The inequalities defining region $\mathrm{IV}^{\bar{w}}$ imply that $\delta^{2-\varepsilon} \rightarrow 0$ and $\delta^{1-\varepsilon+z} \rightarrow 0$ as $\delta \rightarrow 0$. This allows us to compute the asymptotic behavior of (2.57) to leading order. In fact, we conclude from (2.57), (2.58) that

$$
\lim _{\delta \downarrow 0} \frac{\xi^{2} \delta^{2}}{2} Q_{\delta}\left(\frac{t}{\rho^{2}}\right)=\frac{\xi^{2} t \bar{U}^{2} a}{\bar{w}^{2}} \int_{-\infty}^{+\infty} \psi_{0}(k)|k|^{-1-\varepsilon+z} d k .
$$

Therefore, if we define the effective diffusivity

$$
D_{\mathrm{IV} w}^{*}=\frac{\bar{U}^{2} a}{\bar{w}^{2}} \int \psi_{0}(k)|k|^{-1-\varepsilon+z} d k,
$$


we find from (2.27), (2.28) that the solution of the evolution equation with plane-wave initial data $e^{i \xi x+i \eta y}$ converges to

$$
\bar{T}(x, y, t)=e^{i \xi \cdot x+i \eta y} e^{-D_{\mathrm{IV} \dot{w}}^{*} \xi^{2} t} .
$$

This implies that $\bar{T}(x, y, t)$ satisfies, for general initial data, the diffusion equation

$$
\frac{\partial \bar{T}(x, y, t)}{\partial t}=D_{\mathrm{IV} \bar{w}}^{*} \frac{\partial^{2} \bar{T}(x, y, t)}{\partial x^{2}}
$$

II.2.D. Summary. In conclusion, we have obtained in the above analysis four different renormalization regimes for the stratified Gaussian models with nonvanishing transverse mean velocity. We make some further remarks:

(i) The "sweeping effect," or advection of Lagrangian particles transversely to the stratification, produces dominant time decorrelation effects only if $z>1$. In fact, if $\tau_{a}(k)=\left(a|k|^{z}\right)^{-1}$ and $\tau_{\bar{w}}(k)=(|w|(k))^{-1}$ denote respectively the eddy turnover time and the "sweeping" time at wavenumber $k$, we have (for $|k| \ll 1$ )

$$
\tau_{a}(k) \ll \tau_{\bar{w}}(k) \quad \text { if } \quad z<1
$$

and

$$
\tau_{a}(k) \gg \tau_{\bar{w}}(k), \text { if } z>1 .
$$

(ii) The range of validity of mean-field theory is extended by having a nonzero transverse mean flow. In particular, the superdiffusive region $\mathrm{V}$, corresponding to a non-local effective Green function, which exists for $\bar{w}=0$, is not present if $\bar{w} \neq 0$. (iii) The sweeping effect gives rise to a new superdiffusive region, $I^{\bar{w}}$, with an effective equation of diffusion type. The corresponding effective diffusivity, $D_{\mathrm{IV}^{w}}^{*}$, is independent of $D$.

(iv) The phase diagram for $z<1$ coincides with the one for $\bar{w} \neq 0$, since (2.63) holds.

(v) The scaling exponents in the various regions form a continuous function in the $(\varepsilon, z)$ plane, with the exception of the half-line $\varepsilon=2, z>2$, separating regions $\mathrm{I}^{\bar{w}}$ and III $^{\bar{w}}$, along which the exponent jumps from 1 to $1 / 2=1-\left.\frac{\varepsilon}{4}\right|_{\varepsilon=2}$.

(vi) The scaling behavior and the form of the effective equation for $\bar{T}(x, y, t)$ at the boundary between the renormalization regions II $^{\bar{w}}$ and III $^{\bar{w}}$, given by $(0<z<1$, $\varepsilon+2 z=4$ ) is of special interest for applications to turbulence theory. It is studied separately in Sect. IV.

(vii) The remaining portion of the $\varepsilon$, $z$ plane, namely $\varepsilon>4, \varepsilon>0$, constitutes a trivial statistical regime from the point of view of the renormalization problem, in the sense that the system cannot be described by any effective equation on a macroscopic time scale. This is easily seen by observing that for $\varepsilon \geqq 4$, the scaling function for region III $^{\bar{w}}, \rho(\delta)=\delta^{1-\varepsilon / 4}$, does not tend to zero as $\delta \rightarrow 0$.

\section{Statistical Universality: Renormalization for Non-Gaussian Fields}

The assumption made about the Gaussian nature of the fluctuating velocity $u_{\delta}(y, t)$, albeit convenient for calculations, is quite restrictive, and it is interesting to consider 
the renormalization problem for non-Gaussian statistics as well. For instance, it has been argued by some authors $[14,40]$ that the effective Green function or eddydiffusivity, for passive turbulent transport should depend, when super-diffusion occurs, only on the scaling properties of the energy spectrum and the wavenumberdependent turnover time (through $\varepsilon$ and $z$ ), but not on the higher-order statistics of $u$. Our models provide a framework for studying rigorously this hypothesis. Another reason for considering non-Gaussian statistics in the context of stratified models, is the possibility of calculating rigorously the effective equations of motion for some discrete layered models studied previously by Matheron and de Marsily [28], and Bouchaud et al. $[10,11]$.

In this section, we show that the phase diagrams of Figs. 1 and 2 are valid for a wide class of non-Gaussian velocity statistics with power-energy spectrum

$$
E_{\delta}(k, \omega)=\bar{U}^{2} \psi_{0}\left(\frac{k}{\delta}\right) \psi_{\infty}(k) \frac{a^{-1}|k|^{1-\varepsilon-z}}{\pi\left(1+\frac{\omega^{2}}{a^{2}|k|^{2 z}}\right)},
$$

where $\psi_{0}(\cdot), \psi_{\infty}()$ are the standard cutoff functions described in the Introduction. The solution of the renormalization problem given in [1] for $\bar{w}=0$ and in Sect. II for $\bar{w} \neq 0$, relied on the explicit representation formula of the scalar $\langle T(x, y, t)\rangle$ in (2.24), which is valid only for Gaussian fields $u_{\delta}(y, t)$. Here, we develop an alternative approach, which is more general and does not rely on (2.24). Instead, we show that the renormalization problem reduces, after making a suitable change of scale, to the application of a central limit theorem for sums of weakly dependent random variables. Interestingly, despite the non-Fickian superdiffusive scaling functions $\rho(\delta)$ outside regions I and $I^{w}$, we obtain effective equations of motion with effective probability distribution functions for Lagrangian particle displacements which are either purely Gaussian (Regions I, I ${ }^{\bar{w}}$, II, II ${ }^{\bar{w}}$, III, IV IV $^{\bar{w}}$ ), or mixtures of Gaussians, (Region V) with an explicitly given, universal distribution of variances. This characterization is remarkable, given the variety of pdf's which are scale-invariant and consistent with a given scaling function $\rho(\delta)$.

The solution of the renormalization problem hinges on the applicability of limit theorems for sums of dependent random variables. For this reason it is natural to assume certain "mixing" or "weak dependence" statistical hypotheses on the fields $u_{\delta}(y, t)$. Such assumptions are however unnecessary in the mean-field regions I and $I^{\bar{w}}$, which can be handled by one-dimensional, random homogenization methods (cf. Sect. II, Appendix 1, and [1]). These methods require no assumptions on $u_{\delta}(y, t)$ other than statistical homogeneity and a power-energy spectrum of the form (3.1). On the other hand, in the renormalization theory for the superdiffusive regions, mixing assumptions appear to be necessary in order to obtain the same effective equations as in the Gaussian statistics. Here, we do not seek to determine the most general set of assumptions under which the long-time/large distance limit coincides with the one for the Gaussian case. Instead, we focus our attention on two models of random velocity fields, which are natural from the point of view of the theory of disordered systems, and which have some physical significance. They can be associated with complex flows arising from random distributions of momentum sources in a stratified porous medium [28] or with flow in a turbulent shear layer [1]. 
To describe such models, we introduce non-dimensional elementary functions, or "blobs," denoted by $\phi_{\delta}(y, t), \delta>0$, and given by

$$
\phi_{\delta}(y, t)=\frac{1}{2 \pi} \iint \sqrt{E_{\delta}(k, \omega) / \bar{U}^{2}} e^{i k \cdot x+i \omega t} d k d \omega .
$$

Note that, by Plancherel's identity, we have

$$
\iint\left(\phi_{\delta}(y, t)\right)^{2} d y d t=\frac{1}{\bar{U}^{2}} \iint E_{\delta}(k, \omega) d k d \omega .
$$

Moreover, $\phi_{\delta}$ is integrable and satisfies

$$
\iint \phi_{\delta}(y, t) d y d t=0
$$

as a consequence of the vanishing of the Fourier transform of $\phi_{\delta}(y, t)$ in a neighborhood of $k=0$, due to the infrared cutoff $\psi_{0}\left(\frac{k}{\delta}\right)$ in (3.1). To define the first model, we consider a stationary, ergodic point process $\left(p^{(n)}, \tau^{(n)}\right)$ in the plane $\mathbf{R}^{2}$, such that for all open sets $\Omega$ in $\mathbf{R}^{2}$, we have

$$
\lim _{\delta \downarrow 0} \delta^{2} \operatorname{card}\left\{n:\left(\delta p^{(n)}, \delta \tau^{(n)}\right) \in \Omega\right\}=|\Omega|,
$$

where $|\cdot|$ denotes Lebesgue measure. We consider also a sequence of independent, identically distributed random variables $\left\{U_{n}\right\}_{n \geqq 1}$, with zero mean and variance $\bar{U}^{2}$. A stationary random field $u_{\delta}(y, t)$ can be defined by setting

$$
u_{\delta}(y, t)=\sum_{n} U_{n} \phi_{\delta}\left(y+p^{(n)}, t+\tau^{(n)}\right) .
$$

We shall refer to this random velocity field as Model $A$. The second class of models is defined as follows: the point process $\left(p^{(n)}, \tau^{(n)}\right)$ is assumed to be a Poisson point process with unit intensity, thus also satisfying (3.4); (see: for instance, Feller [13]). For this second model, we assume that the $U_{n}$ are identically constant, i.e. $U_{n} \equiv \bar{U}$ for all $n$. This will be referred to as Model B. From the definitions of both models, and the identities (3.3), (3.4), (3.5), it is easy to verify that

$$
\left\langle u_{\delta}(y, t)\right\rangle=0
$$

and that the Eulerian velocity autocorrelation function is given by,

$$
\left\langle u_{\delta}(y, t) u_{\delta}\left(y^{\prime}, t^{\prime}\right)\right\rangle=\iint E_{\delta}(k, \omega) e^{i k\left(y-y^{\prime}\right)+i \omega\left(t-t^{\prime}\right)} d k d \omega .
$$

As models of random velocity fields, Models A and B represent, in a sense, two extremes. In Model A, the components of the fields corresponding to different "sites" $\left(p^{(n)}, \tau^{(n)}\right)$ are uncorrelated for any given realization of the point process $\left(p^{(n)}, \tau^{n}\right)$. On the other hand, in Model $\mathrm{B}$, the Poisson statistics guarantee independence of the components of the velocity field arising from points $\left(p^{(n)}, \tau^{(n)}\right)$ in disjoint regions of the plane. In the theory of disordered systems, Model A is sometimes referred to as having substitutional disorder, and Model B as having translational disorder [26].

Before proceeding to the analysis of the renormalization problem for Models $\mathrm{A}$ 
and B, we mention the well known special case of Model A, given by

$$
\phi_{\delta}(y, t)=\phi(y)=\left\{\begin{array}{lll}
1 & \text { if } & |y|<\frac{1}{2} \\
0 & \text { if } & |y|>\frac{1}{2}
\end{array}\right.
$$

so that $\phi(y)$ is a square pulse of width 1 and height 1 centered at $0, p^{(n)}=n$, for $n=0, \pm 1, \pm 2, \ldots$, and the $U_{n}$ are independent random variables with

$$
\operatorname{Prob}\left\{U_{n}= \pm \bar{U}\right\}=\frac{1}{2}
$$

This model corresponds to a time-independent velocity with energy spectrum

$$
E(k)=\frac{4 \bar{U}^{2} \sin ^{2}(k / 2)}{k^{2}},
$$

and hence to $\varepsilon=1, z=\infty$. It was introduced by Matheron and De Marsily [28]. Physically, the resulting velocity field consists of an infinite array of adjacent horizontal layers with random velocities with values $\pm \bar{U}$. This model has been discussed extensively $[1,3,10,11,13,15]$. Fields analogous to Model B were considered in two and three dimensional models by Koch and Brady [21], Avellaneda and Majda [4], Avellaneda, Torquato and Kim [43] and others [15], to model Darcy velocities in random porous media. In [1], we proposed the Gaussian fields with spectra (3.1) with $\varepsilon=8 / 3$ and $z=2 / 3$ as simple models to study eddy diffusivity in hydrodynamic turbulence. We proceed to the analysis of these models, discussing first the case $\bar{w}=0$ (III.1) and then $\bar{w} \neq 0$ (III.2). As mentioned earlier, the arguments of Sect. II.1 and Appendix 1 for the renormalization in the mean-field region $\mathrm{I}^{\bar{w}}$ (which apply to the case $\bar{w}=0$ as well) require no assumptions on the higher-order statistics and hence are valid without modifications for arbitrary non-Gaussian velocities with spectra of the form (3.1).

III.1. Superdiffusion with Zero Mean Flow $(\bar{w}=0)$. Our starting point is the representation formula for solutions corresponding to plane-wave initial data, (2.23). Making the change of variables $x \rightarrow \frac{x}{\delta}, y \rightarrow \frac{y}{\delta}, t \rightarrow \frac{t}{\rho^{2}}$ and setting $\bar{w}=0$ in that
formula, we obtain

$$
\begin{aligned}
\left\langle T^{\prime}\left(\frac{x}{\delta}, \frac{y}{\delta}, \frac{t}{\rho^{2}}\right)\right\rangle= & \exp \left[i \xi x+i \eta y-\frac{\delta^{2}}{\rho^{2}} \xi^{2} D t\right] \\
& \cdot\left\langle E\left\{\exp \left[i \delta \eta \beta_{2}\left(\frac{t}{\rho^{2}}\right)+i \delta \xi \int_{0}^{t / \rho^{2}} u_{\delta}\left(\frac{y}{\delta}+\sqrt{2 D} \beta_{2}(s), s\right) d s\right]\right\}\right\rangle .
\end{aligned}
$$

Anticipating superdiffusive behavior, i.e., $\delta / \rho \rightarrow 0$ as $\delta \rightarrow 0$, we obtain, to leading order in $\delta$,

$$
\begin{aligned}
\left\langle T^{\prime}\left(\frac{x}{\delta}, \frac{y}{\delta}, \frac{t}{\rho^{2}}\right)\right\rangle & \cong e^{i \xi x+i \eta y}\left\langle E\left\{\exp \left[i \xi \delta \int_{0}^{t / \rho^{2}} u_{\delta}\left(\frac{y}{\delta}+\sqrt{2 D} \beta_{2}(s), s\right) d s\right]\right\}\right\rangle \\
& =e^{i \xi x+i \eta y}\left\langle E\left\{\exp \left[i \xi \delta \int_{0}^{t / \rho^{2}} u_{\delta}\left(\sqrt{2 D} \beta_{2}(s), s\right) d s\right]\right\}\right\rangle
\end{aligned}
$$


Henceforth, we focus our attention on the random variables

$$
\delta \int_{0}^{t / \rho^{2}} u_{\delta}\left(\sqrt{2 D} \beta_{2}(s), s\right) d s
$$

and compute their asymptotic probability distribution as $\delta \rightarrow 0$.

III.1.A. Region II: Central Limit Theorem with Fast-time Decorrelation. Recall that in region II the scaling function is $\rho(\delta)=\delta^{(4-\varepsilon-z) / 2}$. As a first step, we show that the Brownian motion appearing in (3.14) is irrelevant, in the sense that the random variables

$$
\delta \int_{0}^{t / \rho^{2}} u_{\delta}(0, s) d s
$$

have the same asymptotic probability distribution as the ones in (3.14). In fact, we have

$$
\begin{aligned}
\langle E & \left.\left\{\left.\left|\delta \int_{0}^{t / \rho^{2}} u_{\delta}\left(\sqrt{2 D} \beta_{2}(s), s\right) d s-\delta \int_{0}^{t / \rho^{2}} u_{\delta}(0, s) d s\right|\right|^{2}\right\}\right\rangle \\
= & \frac{\bar{U}^{2} t^{2} \delta^{4-\varepsilon}}{\rho^{4}} \iint \frac{\psi_{0}(k) \psi_{\infty}(\delta k)|k|^{1-\varepsilon-z}}{\pi a\left[1+\frac{\omega^{2}}{a^{2}|k|^{2 z}}\right]} \\
& \cdot\left[\int_{0}^{1} \int_{0}^{1} 2\left(1-e^{\left.-\left(D|k|^{2} \delta^{2} t\left(s-s^{\prime}\right)\right) / \rho^{2}\right)} e^{\left(i \omega t \delta^{z}\left(s-s^{\prime}\right)\right) / \rho^{2}} d s d s^{\prime}\right] d k d \omega\right. \\
= & \frac{2 \bar{U}^{2} t^{2} \delta^{4-\varepsilon}}{\rho^{4}} \int \psi_{0}(k) \psi_{\infty}(\delta k)|k|^{1-\varepsilon}\left[\int _ { 0 } ^ { 1 } \int _ { 0 } ^ { 1 } \left(1-e^{\left.\left.-\left(D|k|^{2} \delta^{2} t\left(s-s^{\prime}\right)\right) / \rho^{2}\right) e^{-\left(a|k|^{z} \delta^{z} t\left(s-s^{\prime}\right)\right) / \rho^{2}}\right] d k} .\right.\right.
\end{aligned}
$$

Since $1-e^{-\left(D|k|^{2} \delta^{2} t\left(s-s^{\prime}\right)\right) / \rho^{2}}$ converges to zero as $\delta \rightarrow 0$ for all $k, s, s^{\prime}$ and, moreover, the integral

$$
\begin{aligned}
& \frac{2 \bar{U}^{2} t^{2} \delta^{4-\varepsilon}}{\rho^{4}} \int \psi_{0}(k) \psi_{\infty}(\delta k)\left(\int_{0}^{1} \int_{0}^{1} e^{-\left(a|k| z \delta z t\left(s-s^{\prime}\right)\right) / \rho^{2}} d s d s^{\prime}\right)|k|^{1-\varepsilon} d k \\
& \sim \frac{2 \bar{U}^{2} t}{a}\left[\int \psi_{0}(k)|k|^{1-\varepsilon-z} d k+o(1)\right]
\end{aligned}
$$

is uniformly bounded as $\delta \rightarrow 0$, we conclude that

$$
\left\langle E\left\{\left|\delta \int_{0}^{t / \rho^{2}} u_{\delta}\left(\sqrt{2 D} \beta_{2}(s), s\right)-\delta \int_{0}^{t / \rho^{2}} u_{\delta}(0, s) d s\right|^{2}\right\}\right\rangle
$$

tends to zero as $\delta \rightarrow 0$, and hence (3.14) and (3.15) have the same asymptotic distribution.

The second step is to show that (3.15) satisfies an appropriate Central Limit Theorem. For this, define the "spectral measure"

$$
d Z(k, \omega)=\left(\sum_{n} U_{n} e^{+i k p^{(n)}+i \omega \tau^{(n)}}\right) d k d \omega,
$$


and note that the field, $u_{\delta}(0, s)$ satisfies

$$
u_{\delta}(0, s)=\delta^{(2-\varepsilon) / 2} \frac{1}{2 \pi} \iint \frac{\psi_{0}^{1 / 2}(k) \psi_{\infty}^{1 / 2}(\delta k)|k|^{(1-\varepsilon-z) / 2}}{\pi^{1 / 2} a^{1 / 2}\left[1+\frac{\omega^{2}}{a|k|^{2 z}}\right]^{1 / 2}} e^{i \omega \delta^{z} s} \frac{d Z\left(\delta k, \delta^{z} \omega\right)}{\delta^{(1+z) / 2}} .
$$

Therefore, if we define a new field $V_{\delta}(s)$ by

$$
V_{\delta}(s)=\frac{\rho}{\delta^{z / 2}} u_{\delta}\left(0, \frac{s}{\delta^{z}}\right)=\frac{1}{2 \pi} \iint \frac{\psi_{0}^{1 / 2}(k) \psi_{\infty}^{1 / 2}(\delta k)|k|^{(1-\varepsilon-z) / 2}}{\pi^{1 / 2} a^{1 / 2}\left[1+\frac{\omega^{2}}{a|k|^{2 z}}\right]^{1 / 2}} e^{i \omega s} \frac{d Z\left(\delta k, \delta^{z} \omega\right)}{\delta^{(1+z) / 2}}
$$

and the intermediate time-scale $\theta(\delta)$ by

we have

$$
\theta(\delta)=\frac{t \delta^{z}}{\rho^{2}}=t \delta^{2 z+\varepsilon-4}
$$

$$
\delta \int_{0}^{t / \rho^{2}} u_{\delta}(0, s) d s=\frac{t^{1 / 2}}{\sqrt{\theta(\delta)}} \int_{0}^{\theta(\delta)} V_{\delta}(s) d s
$$

Notice that $\theta(\delta) \rightarrow \infty$ as $\delta \rightarrow 0$, and that the covariance of the right-hand side,

$$
\left\langle\left|\frac{1}{\sqrt{\theta(\delta)}} \int_{0}^{\theta(\delta)} V_{\delta}(s) d s\right|^{2}\right\rangle
$$

remains uniformly bounded as $\delta \rightarrow 0$, due to our choice of $\rho(\delta)$ (cf. Sect. II.1.A and Eq. (3.17)). It is known that, under these circumstances, $\frac{1}{\sqrt{\theta(\delta)}} \int_{0}^{\theta(\delta)} V_{\delta}(s) d s$ is asymptotically Gaussian, provided $V_{\delta}(s)$ satisfies suitable statistical mixing conditions. To show asymptotic normality, we use a central limit theorem for velocities having "blob structure," proved in Appendix 2. A possible alternative approach, (not pursued here) would have consisted in verifying that the fields $V_{\delta}(s)$ satisfy a specific set of standard mixing assumptions such as those in Ibragimov [18], Billingsley [8], Kesten and Papanicolaou [19] or others [9,20].

Observe from (3.21) that the velocity field $V_{\delta}(s)$ has the form

where

$$
V_{\delta}(s)=\delta^{(1+z) / 2} \sum_{n} U_{n} G_{\delta}\left(\delta \rho^{(n)}, s+\delta^{z} \tau^{(n)}\right),
$$

$$
G_{\delta}(y, s)=\frac{1}{2 \pi} \iint \frac{\psi_{0}^{1 / 2}(k) \psi_{\infty}(\delta k)^{1 / 2}|k|^{(1-\varepsilon-z) / 2}}{a^{1 / 2} \pi^{1 / 2}\left(1+\frac{\omega^{2}}{a|k|^{2 z}}\right)^{1 / 2}} e^{i k y+i \omega s} d k d \omega .
$$

Using (3.25), we apply Proposition B.3, in Appendix 2, noting that the elementary "blobs" $G_{\delta}(y, s)$ satisfy

$$
\iint G_{\delta}(y, s) d y d s=0
$$

for each $\delta>0$, so that

$$
\left\langle\frac{1}{\sqrt{\theta(\delta)}} \int_{0}^{\theta(\delta)} V_{\delta}(s) d s\right\rangle=0
$$


and, moreover,

$$
\begin{aligned}
\lim _{\delta \downarrow 0}\left\langle\left|\frac{1}{\sqrt{\theta(\delta)}} \int_{0}^{\theta(\delta)} V_{\delta}(s) d s\right|^{2}\right\rangle & =\left.\left.2 \lim _{\delta \downarrow 0} \int_{-\infty}^{+\infty} d y\right|_{-\infty} ^{+\infty} G_{\delta}(y, s) d s\right|^{2} \\
& =\frac{2 \bar{U}^{2}}{a} \int \psi_{0}(k)|k|^{1-\varepsilon-z} d k .
\end{aligned}
$$

From Proposition B.3, we conclude that $\frac{1}{\sqrt{\theta(\delta)}} \int_{0}^{\theta(\delta)} V_{\delta}(s) d s$ converges in distribution to a Gaussian random variable with mean zero and variance

$$
2 D_{\mathrm{II}}^{*}=\frac{2 \bar{U}^{2}}{a} \int \psi_{0}(k)|k|^{1-\varepsilon-z} d k .
$$

Using (3.13), we see that the effective equation corresponding to region II for Models A and B is

$$
\frac{\partial \bar{T}(x, y, t)}{\partial t}=D_{\mathrm{II}}^{*} \frac{\partial^{2} \bar{T}(x, y, t)}{\partial x^{2}}
$$

and in the Gaussian case.

III.1.B. Region III. Recall that the scaling function for region III is $\rho(\delta)=\delta^{1-\varepsilon / 4}$. As in the preceding paragraph, the renormalization problem reduces to determining the asymptotic distribution for the random variables

$$
\delta \int_{0}^{t / \rho^{2}} U_{\delta}\left(\sqrt{2 D} \beta_{2}(s), s\right) d s
$$

as $\delta \rightarrow 0$. Again, since $\delta \gg \rho(\delta)$, the contribution from the Brownian motion is negligible, and (3.32) is asymptotically equivalent to

$$
\delta \int_{0}^{t / \rho^{2}} u_{\delta}(0, s) d s
$$

More precisely, we have

$$
\begin{aligned}
& \left\langle E\left\{\left|\delta \int_{0}^{t / \rho^{2}} u_{\delta}\left(\sqrt{2 D} \beta_{2}(s), s\right) d s-\delta \int_{0}^{t / \rho^{2}} u_{\delta}(0, s) d s\right|^{2}\right\}\right\rangle \\
& =2 t^{2} \bar{U}^{2} \int \psi_{0}(k) \psi_{\infty}(\delta k)|k|^{1-\varepsilon}\left[\int_{0}^{1} \int_{0}^{1}\left(1-e^{-\left(D|k|^{2} \delta^{2} t\left(s-s^{\prime}\right)\right) / \rho^{2}}\right) e^{-\left(a|k|^{z} \delta z t\left(s-s^{\prime}\right)\right) / \rho^{2}} d s d s^{\prime}\right] d k,
\end{aligned}
$$

a quantity converging to zero as $\delta \rightarrow 0$, by the Dominated Convergence Theorem. Having reduced the problem to (3.33), we introduce the auxiliary "blob" function

$$
G_{\delta}(y, s)=\frac{t}{2 \pi} \iint \frac{\psi_{0}^{1 / 2}(k) \psi_{\infty}^{1 / 2}(k)|k|^{(1-\varepsilon-z) / 2}}{a^{1 / 2} \pi^{1 / 2}\left(1+\frac{\omega^{2}}{a^{2}|k|^{2 z}}\right)^{1 / 2}}\left(\int_{0}^{1} e^{\left(i \omega \delta^{z} t s\right) / \rho^{2}} d s\right) e^{i k y+i \omega s} d k d \omega
$$


Using the function $G_{\delta}(y, s)$, we can rewrite the integral (3.33) in the form

$$
\delta \int_{0}^{t / \rho^{2}} u_{\delta}(0, s) d s=\delta^{(1+z) / 2} \sum_{n} U_{n} G_{\delta}\left(\delta p^{(n)}, \delta^{z} \tau^{(n)}\right) .
$$

The blobs $G_{\delta}(y, s)$ satisfy

for each $\delta \rightarrow 0$ and

$$
\iint G_{\delta}(y, s) d y d s=0
$$

where

$$
\lim _{\delta \downarrow 0} \iint\left|G_{\delta}(y, s)-G_{0}(y, s)\right|^{2} d y d s=0
$$

$$
G_{0}(y, s)=\frac{t}{2 \pi} \iint \frac{\psi_{0}^{1 / 2}(k)|k|^{(1-\varepsilon-z) / 2}}{a^{1 / 2} \pi^{1 / 2}\left(1+\frac{\omega^{2}}{a^{2}|k|^{2 z}}\right)^{1 / 2}} e^{+(i k y+i \omega s)} d k d \omega
$$

This function satisfies, by Plancherel's identity,

$$
\iint\left(G_{0}(y, s)\right)^{2} d y d s=t^{2} \int \psi_{0}(k)|k|^{1-\varepsilon} d k .
$$

We can thus apply Proposition B.2, in Appendix 2 to conclude that for Models A and $\mathrm{B}$, the random variables $\delta \int_{0}^{t / \rho^{2}} u_{\delta}(0, s) d s$ converge in distribution as $\delta \rightarrow 0$, to a centered Gaussian with variance

$$
\bar{U}^{2} t^{2} \int \psi_{0}(k)|k|^{1-\varepsilon} d k
$$

By the usual argument, this implies that the effective equation for region III for Models A and B is

with

$$
\frac{\partial \bar{T}(x, y, t)}{\partial t}=D_{\mathrm{III}}^{*}(t) \frac{\partial^{2} \bar{T}(x, y, t)}{\partial x^{2}}
$$

$$
D_{\mathrm{III}}^{*}(t)=\frac{\bar{U}^{2} t}{2} \int \psi_{0}(k)|k|^{1-\varepsilon} d k
$$

This result agrees with the characterization of region III for Gaussian statistics obtained in [1].

III.1.C. Region $I V$. We follow the general procedure of the above two paragraphs, with $\rho(\delta)=\delta^{z /(\varepsilon+2 z-2)}$. As before, $\delta \int_{0}^{t / \rho^{2}} u_{\delta}\left(\sqrt{2 D} \beta_{2}(s), s\right) d s$ is asymptotically equivalent to $\delta \int_{0}^{t / \rho^{2}} u_{\delta}(0, s) d s$ as $\delta \rightarrow 0$. The latter integral can be expressed as a sum of "blobs," evaluated at the points $\left\{\left(\delta p^{(n)}, \delta^{z} \tau^{(n)}\right)\right\}, n \geqq 0$, multiplied by the "amplitudes" $\left\{U_{n}\right\}, n \geqq 0$. In fact, let $G_{\delta}(y, s)$ be defined as

$$
G_{\delta}(y, s)=\frac{t}{2 \pi} \iint \frac{\psi_{0}\left(\frac{g k}{\delta}\right)^{1 / 2} \psi_{\infty}(g k)^{1 / 2}|k|^{(1-\varepsilon-z) / 2}}{a^{1 / 2} \pi^{1 / 2}\left(1+\frac{\omega^{2}}{a^{2}|k|^{2 z}}\right)^{1 / 2}}\left(\frac{e^{i \omega t}-1}{i \omega}\right) e^{i \omega s+i k y} d k d \omega
$$


where $g$ is an "intermediate" scaling function, given by

$$
g=g(\delta)=\delta^{2 /(2 z+\varepsilon-2)} \text {. }
$$

Notice that $\lim g(\delta)=0$ in region IV. With these definitions, it is easy to check that $\delta \downarrow 0$

$$
\delta \int_{0}^{t / \rho^{2}} u_{\delta}(0, s) d s=(g(\delta))^{(1+z) / 2} \sum_{n} U_{n} G_{\delta}\left((g(\delta)) p^{(n)},(g(\delta))^{z} \tau^{(n)}\right) .
$$

It can also be verified that $\iint G_{\delta}(y, s) d y d s=0$ and that $G_{\delta}(y, s)$ converges strongly in $L^{2}\left(\mathbf{R}^{2}\right)$ to $G_{0}(y, s)$ as $\delta \rightarrow 0$, where

$$
G_{0}(y, s)=\frac{t}{2 \pi} \iint \frac{|k|^{(1-\varepsilon-z) / 2}}{a^{1 / 2} \pi^{1 / 2}\left(1+\frac{\omega^{2}}{a^{2}|k|^{2 z}}\right)^{1 / 2}}\left(\frac{e^{i \omega t}-1}{i \omega t}\right) e^{i k y+i \omega s} d k d \omega .
$$

From Plancherel's Identity, we obtain

$$
\begin{aligned}
\iint\left(G_{0}(y, s)\right)^{2} d y d s & =t^{2} \iint \frac{|k|^{1-\varepsilon-z}}{a \pi\left(1+\frac{\omega^{2}}{a|k|^{2 z}}\right)}\left|\int_{0}^{1} e^{i \omega t s} d s\right|^{2} d k d \omega \\
& =t^{2} \int|k|^{1-\varepsilon}\left(\int_{0}^{1} \int_{0}^{1} e^{-a|k|^{z} t\left(s-s^{\prime}\right)} d s d s^{\prime}\right) d k \\
& =2 t^{2}(a t)^{(\varepsilon-2) / z} \int|k|^{1-\varepsilon-z}\left(1-\frac{1-e^{-|k|^{z}}}{|k|^{z}}\right) d k .
\end{aligned}
$$

Applying Proposition B.2 in Appendix 2, we conclude that $\delta \int_{0}^{t / \rho^{2}} u_{\delta}(0, s) d s$ converges in distribution to a normal random variable with mean zero and variance

$$
\frac{2 \bar{U}^{2}}{a} t(a t)^{1+(\varepsilon-2) / z} \int_{-\infty}^{+\infty}|k|^{1-\varepsilon-z}\left(1-\frac{1-e^{-|k|^{z}}}{|k|^{z}}\right) d k .
$$

Differentiating this expression with respect to $t$, we conclude that the effective equation in region IV for Models A, B is

$$
\frac{\partial \bar{T}(x, y, t)}{\partial t}=D_{\mathrm{IV}}^{*}(t) \frac{\partial^{2} \bar{T}(x, y, t)}{\partial x^{2}}
$$

where the effective diffusivity is given by

$$
D_{\mathrm{IV}}^{*}(t)=\left(2+\frac{\varepsilon-2}{z}\right)(a t)^{1+(\varepsilon-2) / z} \frac{\bar{U}^{2}}{a} \int_{-\infty}^{+\infty}|k|^{1-\varepsilon-z}\left(1-\frac{1-e^{-|k|^{z}}}{|k|^{z}}\right) d k .
$$

III.1.D. Region $V$. The time-scaling function in region $\mathrm{V}$, from [1], is given by $\rho(\delta)=\delta^{1 /(1+\varepsilon / 2)}$. Unlike in the other superdiffusive regions, Brownian motion is not irrelevant in region $\mathrm{V}$. In fact, the fluctuations in the Lagrangian velocity caused by sampling the field with a Brownian motion dominate the fluctuations arising from statistical time-decorrelation. This can be understood by comparison 
of the turnover time $\tau_{a}(k)=\left(a|k|^{2}\right)^{-1}$ and the diffusion time $\tau_{D}(k)=\left(D|k|^{2}\right)^{-1}$. In fact, if $z>2$, we have $\tau_{D}(k) \ll \tau_{a}(k)$ for $|k| \ll 1$. As before, we consider the random variables $\delta \int_{0}^{t / \rho^{2}} u_{\delta}\left(\sqrt{2 D} \beta_{2}(s), s\right) d s$ as $\delta \rightarrow 0$. We introduce the auxiliary "blob" functions

$$
\begin{aligned}
G_{\delta}(y, s)= & \frac{t^{(2+\varepsilon) / 4}}{(2 D)^{(2-\varepsilon) / 4}} \frac{1}{2 \pi} \iint \frac{\psi_{0}\left(\frac{\rho k}{\delta}\right)^{1 / 2} \psi_{\infty}(\rho k)^{1 / 2}|k|^{(1-\varepsilon-z) / 2}}{a^{1 / 2} \pi^{1 / 2}\left(1+\frac{\omega^{2}}{a^{2}|k|^{2 z}}\right)^{1 / 2}} \\
& \cdot\left(\int_{0}^{1} e^{i\left(\omega \rho^{z-2} t s\right) /(2 D t)^{z / 2}+i k \tilde{\beta}(s)} d s\right) e^{i(k y+\omega s)} d k d \omega,
\end{aligned}
$$

where $\tilde{\beta}(s) \equiv \frac{\rho}{t^{1 / 2}} \beta_{2}\left(\frac{t}{\rho^{2}} s\right)$ is distributed like a Brownian motion on the interval $(0,1)$. With the functions $G_{\delta}(y, s)$, we can write

$$
\delta \int_{0}^{t / \rho^{2}} u_{\delta}\left(\sqrt{2 D} \beta_{2}(s), s\right) d s=\rho^{(1+z) / 2} \sum_{n} U_{n} G_{\delta}\left(\rho p^{(n)}, \rho^{z} \tau^{(n)}\right) .
$$

We apply Proposition B.2, Appendix 2, noting that that the functions $G_{\delta}(y, s)$ defined in (3.52) satisfy the necessary requirements. In fact, we have

$$
\iint\left(G_{\delta}(y, s)\right) d y d s=0,
$$

and the sequence $\left\{G_{\delta}(y, s)\right\}$ is compact in $L^{2}\left(\mathbf{R}^{2}\right)$, almost surely with respect to $\tilde{\beta}(s)$, $0<s<1$. This can be seen as follows: for each $\delta>0$, we have

$$
\begin{aligned}
& \iint\left(G_{\delta}(y, s)\right)^{2} d y d s \\
& \quad=\frac{t^{(1+\varepsilon) / 2}}{(2 D)^{(1-\varepsilon) / 2}} \iint \frac{\psi_{0}\left(\frac{\rho k}{\delta}\right) \psi_{\infty}(\rho k) k^{1-\varepsilon-z}}{a \pi\left(1+\frac{\omega^{2}}{a^{z}|k|^{2 z}}\right)}\left|\int_{0}^{1} \exp \left[\frac{i \omega \rho^{z-2} t s}{(2 D t)^{z / 2}}+i k \tilde{\beta}(s)\right] d s\right|^{2} d k d \omega .
\end{aligned}
$$

Notice that in this integral $\rho^{z-2} \ll 1$ as $\delta \rightarrow \infty$. Moreover, it follows from Lemma (5.1) in [1], that, except for a set of realizations of $\widetilde{\beta}(\cdot)$ of Wiener measure zero, the integrals in (3.55) are uniformly bounded for $0 \leqq \delta<1$ (with a bound depending on the path $\widetilde{\beta}(\cdot))$. Applying Lemma (5.1) [1], together with the Dominated Convergence Theorem we find that, with probability $1, G_{\delta}(y, s)$ converges strongly in $L^{2}\left(\mathbf{R}^{2}\right)$ to $G_{0}(y, s)$, where

$$
G_{0}(y, s)=\frac{1}{2 \pi} \frac{t^{(2+\varepsilon) / 4}}{(2 D)^{(2-\varepsilon) / 4}} \iint \frac{|k|^{(1-\varepsilon-z) / 2}}{a^{1 / 2} \pi^{1 / 2}\left(1+\frac{\omega^{2}}{a^{2}|k|^{2 z}}\right)^{1 / 2}}\left(\int_{0}^{1} e^{i k \tilde{\beta}(s)} d s\right) e^{i(k y+\omega s)} d k d \omega .
$$


We conclude from these facts and Proposition B.2, Appendix 2, that

$$
\begin{aligned}
\lim _{\delta \downarrow 0} & \left\langle\exp \left[i \xi \delta \int_{0}^{t / \rho^{2}} u_{\delta}\left(\sqrt{2 D} \beta_{2}(s), s\right) d s\right]\right\rangle \\
& =\lim _{\delta \downarrow 0}\left\langle\exp \left[i \xi \delta \int_{0}^{t / \rho^{2}} u_{\delta}\left(\frac{\sqrt{2 D t}}{\rho} \tilde{\beta}\left(\frac{s \rho^{2}}{t}\right)\right) d s\right]\right\rangle \\
& =\lim _{\delta \downarrow 0}\left\langle\exp \left[i \frac{\xi t \delta}{\rho^{2}} \int_{0}^{1} u_{\delta}\left(\frac{\sqrt{2 D t}}{\rho} \tilde{\beta}(s)\right) d s\right]\right\rangle \\
& =\exp \left[-\frac{\xi^{2} t^{1+\varepsilon / 2} \bar{U}^{2}}{2(2 D)^{1-\varepsilon / 2}} \alpha_{\varepsilon}(\tilde{\beta})\right], \tilde{\beta}(\cdot)-\text { almost surely, }
\end{aligned}
$$

where $\alpha_{\varepsilon}(\widehat{\beta})$ is a random variable that depends on the path $\hat{\beta}$, given by

$$
\alpha_{\varepsilon}(\widehat{\beta})=\int_{-\infty}^{+\infty}|k|^{1-\varepsilon}\left|\int_{0}^{1} e^{i k \hat{\beta}(s)} d s\right|^{2} d k
$$

Consequently, the Fourier transform of the effective probability distribution function, or Green function, is given by

$$
\bar{T}(\xi, t)=E\left\{\exp \left[-\frac{t^{1+\varepsilon / 2 \bar{U}^{2}}}{2(2 D)^{1-\varepsilon / 2}}|\xi|^{2} \alpha_{\varepsilon}(\hat{\beta})\right]\right\}=\int_{0}^{\infty} \exp \left[-\frac{t^{1+\varepsilon / 2}|\xi|^{2}}{2(2 D)^{1-\varepsilon / 2}} \alpha\right] d \tilde{v}_{\varepsilon}(\alpha),
$$

where $\tilde{v}_{\varepsilon}(\alpha)$ denotes the probability distribution function of the random variable $\alpha_{\varepsilon}(\widetilde{\beta})$. This result coincides with the renormalization in region V with Gaussian statistics derived in [1].

III.2. The Case $\bar{w} \neq 0$. The renormalization theory with $\bar{w} \neq 0$ and non-Gaussian statistics follows the same methods as for the case $\bar{w}=0$. For simplicity, we omit detailed calculations. What follows is a brief analysis of the three different superdiffusive regions: II $^{\bar{w}}, \mathrm{III}^{\bar{w}}$, and $\mathrm{IV}^{\bar{w}}$.

Region $I I^{\bar{w}}$. The renormalization follows the same ideas as in Sect. (II.1.A), for region II, the starting point being the integrals

$$
\delta \int_{0}^{t / \rho^{2}} u_{\delta}\left(\sqrt{2 D} \beta_{2}(s)+\bar{w} s, s\right) d s
$$

It can be shown, using the scaling function $\rho(\delta)=\delta^{(4-\varepsilon-z) / 2}$ that in region II $^{\bar{w}}$ the random variables in (3.60) are asymptotically equivalent to

$$
\delta \int_{0}^{t / \rho^{2}} u_{\delta}(0, s) d s
$$

i.e. the Brownian motion and the shift induced by the transverse mean field are negligible relative to the fast time decorrelation of the velocity field. The proof then follows exactly the one for $\bar{w}=0$ of III.1.A and the effective equation of motion coincides with the one in (3.31).

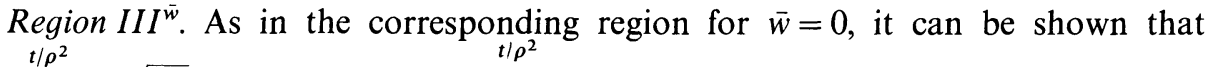
$\delta \int_{0}^{t / \rho^{2}} u_{\delta}\left(\sqrt{2 D} \beta_{2}(s)+\bar{w} s, s\right) d s$ and $\delta \int_{0}^{t / \rho^{2}} u_{\delta}(0, s) d s$ are asymptotically equivalent. Thus, 
repeating the argument of III.1 we find that the scaling function and the effective equation of motion coincides with the one for $\bar{w}=0$; the latter is given in (3.42).

Region $I V^{\bar{w}}$. This is a superdiffusive region in the phase diagram, in which the "sweeping effect" from the transverse mean flow is important. The corresponding calculation for Gaussian statistics, given in Sect. II.2.C, yields a time-scaling function $\rho(\delta)=\delta^{1-(\varepsilon-z) / 2}$. Since $z<2$, we expect that Brownian effects will be negligible. In fact, it can be shown that the random variables

$$
\delta \int_{0}^{t / \rho^{2}} u_{\delta}\left(\sqrt{2 D} \beta_{2}(s)+\bar{w} s, s\right) d s \text { and } \delta \int_{0}^{t / \rho^{2}} u_{\delta}(\bar{w} s, s) d s
$$

have the same asymptotic distribution. We omit this straightforward calculation. To investigate the asymptotic distribution of the second integral in (3.62) we define the auxiliary field $V_{\delta}(s)$ by

$$
V_{\delta}(s)=\frac{\delta^{1 / 2}}{\rho} u_{\delta}\left(\frac{\bar{w} s}{\delta}, \frac{s}{\delta}\right) .
$$

Introducing the intermediate time-scale

$$
\theta(\delta) \equiv \frac{\delta t}{\rho^{2}}=\frac{t}{\delta^{1+z-\varepsilon}}
$$

we have

$$
\delta \int_{0}^{t / \rho^{2}} u_{\delta}(\bar{w} s, s) d s=\frac{t^{1 / 2}}{\sqrt{\theta(\delta)}} \int_{0}^{\theta(\delta)} V_{\delta}(s) d s
$$

Notice that $\theta(\delta) \rightarrow \infty$ for $\delta \rightarrow 0$. Thus, the right-hand side of (3.65) is a normalized sum of dependent random variables. To show that it is asymptotically Gaussian, we introduce the auxiliary "blob"

$$
G_{\delta}(y, s)=\frac{1}{2 \pi} \iint \hat{G}_{\delta}(k, \omega) e^{i k y+i \omega s} d k d \omega
$$

with $\hat{G}_{\delta}(k, \omega)$ given by

$$
\hat{G}_{\delta}(k, \omega)=\frac{a^{1 / 2} \psi_{0}(k)^{1 / 2}|k|^{(1-\varepsilon+z) / 2}}{\pi^{1 / 2}\left[a^{2} \delta^{2(z-1)}|k|^{2 z}+\omega^{2}\right]^{1 / 2}} .
$$

A straightforward calculation shows that the field $V_{\delta}(s)$ is a superposition of randomly shifted blobs $G_{\delta}(y, s)$. Specifically, we have

$$
V_{\delta}(s)=\delta^{2} \sum_{n} U_{n} G_{\delta}\left(\bar{w} s+\delta p^{(n)}, s+\delta \tau^{(n)}\right) .
$$

Applying Proposition B.4, Appendix 2, we conclude that $\frac{1}{\sqrt{\theta(\delta)}} \int_{0}^{\theta(\delta)} V_{s}(s)$ is asymptotically Gaussian, with mean zero and variance

$$
\sigma^{2}=\lim _{\delta \downarrow 0}\left\langle\left.\frac{1}{\sqrt{\theta(\delta)}} \int_{0}^{\theta(\delta)} V_{\delta}(s) d s\right|^{2}\right\rangle=\frac{2 \bar{U}^{2} a}{\bar{w}^{2}} \int_{-\infty}^{+\infty} \psi_{0}(k)|k|^{-1-\varepsilon+z} d k
$$


Therefore, the effective equation of motion in region $I V^{\bar{w}}$ is the diffusion equation

$$
\frac{\partial \bar{T}(x, y, t)}{\partial t}=D_{\mathrm{IV} \overline{\mathrm{w}}}^{*} \frac{\partial^{2} \bar{T}(x, y, t)}{\partial x^{2}}
$$

with

$$
D_{\mathrm{IV} \dot{w}}^{*}=\frac{\bar{U}^{2} a}{\bar{w}^{2}} \int_{-\infty}^{+\infty} \psi_{0}(k)|k|^{-1-\varepsilon+z} d k
$$

This result coincides with the one derived in II.2.C for Gaussian statistics.

\section{The Boundaries Between Regions II and III, or II $^{\bar{w}}$ and III ${ }^{\bar{w}}$}

It is particularly interesting to study the intermediate regime between II and III or, equivalently, II $^{\bar{w}}$ and III $^{\bar{w}}$ because of their relevance to the statistical theory of turbulence. According to the Kolmogorov-Obukhov " $k^{-5 / 3}$ law" $[22,32]$, the spectral parameters associated with homogeneous turbulence are $\varepsilon=8 / 3$ and $z=2 / 3$, and hence correspond precisely to a point lying on the boundary between II and III, or II ${ }^{\bar{w}}$ and III $^{\bar{w}}$, i.e., to the line segment

$$
\varepsilon+2 z=4,0<z<1 \text {. }
$$

Notice that for values of the parameter $z$ in the range $0<z<1$, the time decorrelation induced by sampling of the random field with a transversal mean velocity (sweeping effect), is negligible compared to the Eulerian time-decorrelation effect, and consequently, the corresponding effective equations are the same whether $\bar{w}$ vanishes or not.

As we shall demonstrate hereafter, if (4.1) holds, the system is superdiffusive with scaling function

$$
\rho(\delta)=\delta^{(4-\varepsilon-z) / 2}
$$

and the effective equation of motion is

$$
\frac{\partial \bar{T}(x, y, t)}{\partial t}=D^{*}(t) \frac{\partial^{2} \bar{T}(x, y, t)}{\partial x^{2}},
$$

where $D^{*}(t)$ is a time-dependent diffusivity satisfying $D^{*}(t) \sim D_{\mathrm{III}}^{*}(t)$ for $t \ll 1$ and $D^{*}(t) \sim D_{\mathrm{II}}^{*}$ for $t \gg 1$. The scaling function is identical to the one for regions II and II $^{\bar{w}}$.

With the choice of (4.2) for the scaling function, we consider the integral

$$
\delta \int_{0}^{t / \rho^{2}} u_{\delta}\left(\sqrt{2 D} \beta_{2}(s)+\bar{w} s, s\right) d s .
$$

We wish to compute the asymptotic distribution of this quantity as $\delta \rightarrow 0$. A straightforward variance calculation (as in (3.16)-(3.17)), shows that (4.4) and

$$
\delta \int_{0}^{t / \rho^{2}} u_{\delta}(0, s) d s
$$

have the same asymptotic probability distribution as $\delta \downarrow 0$, and hence it is sufficient 
to study (4.5). As in III.1.A, we define the auxiliary velocity

$$
V_{\delta}(s)=\frac{\rho}{\delta^{z / 2}} u_{\delta}\left(0, \frac{s}{\delta^{z}}\right)
$$

Comparison with the arguments in III.1.A, shows that the intermediate time scale in (3.22), given by

$$
\theta(\delta)=\frac{t \delta^{2}}{\rho^{2}}=t \delta^{2 z-\varepsilon-4}=t
$$

is independent of $\delta$, remaining of order 1 , because $2 z+\varepsilon=4$. We obtain accordingly, from (3.21), (3.22), (3.23) that

$$
\delta \int_{0}^{t / \rho^{2}} u_{\delta}(0, s) d s=\int_{0}^{t} V_{\delta}(s) d s
$$

If the random velocity $u_{\delta}(y, t)$ corresponds to Models $\mathrm{A}$ or $\mathrm{B}$, then the integral on the right-hand side of (4.8) can be expressed as a sum of "blobs," i.e.,

$$
\int_{0}^{t} V_{\delta}(s) d s=\delta^{(1+z) / 2} \sum_{n} U_{n} G_{\delta}\left(\delta p^{(n)}, \delta^{z} \tau^{(n)}\right)
$$

where

$$
G_{\delta}(y, s)=\frac{1}{2 \pi} \iint \frac{\psi_{0}^{1 / 2}(k) \psi_{\infty}^{1 / 2}(\delta k)|k|^{(1-\varepsilon-z) / 2}}{a^{1 / 2} \pi^{1 / 2}\left[1+\frac{\omega^{2}}{a^{2}|k|^{2 z}}\right]^{1 / 2}}\left[\int_{0}^{t} e^{i \omega \sigma} d \sigma\right] e^{i k y+i \omega s} d k d \omega
$$

This function satisfies the assumptions of Proposition B.2, Appendix 2, namely

$$
\int_{-\infty}^{+\infty} \int_{-\infty}^{+\infty} G_{\delta}(y, s) d y d s=0
$$

for each $\delta>0$ and $G_{\delta}(y, s)$ converges strongly in $L^{2}\left(\mathbf{R}^{2}\right)$ to $G_{0}(y, s)$ given by

$$
G_{0}(y, s)=\frac{1}{2 \pi} \iint \frac{\psi_{0}^{1 / 2}(k)|k|^{(1-\varepsilon-z) / 2}}{a^{1 / 2} \pi^{1 / 2}\left(1+\frac{\omega^{2}}{a^{2}|k|^{2 z}}\right)^{1 / 2}}\left(\int_{0}^{t} e^{i \omega \sigma} d \sigma\right) e^{i k y+i \omega s} d k d \omega .
$$

The $L^{2}$-norm of $G_{0}(y, s)$ can be calculated by Plancherel's formula:

$$
\begin{aligned}
\int_{-\infty}^{+\infty} \int_{-\infty}^{+\infty}\left(G_{0}(y, s)\right)^{2} d y d s & =\iint \frac{\psi_{0}(k)|k|^{1-\varepsilon-t}}{a \pi\left(1+\frac{\omega^{2}}{a^{2}|k|^{2 z}}\right)}\left(\int_{0}^{t} \int_{0}^{t} e^{i \omega(\sigma-\sigma)} d \sigma d \sigma^{\prime}\right) d k d \omega \\
& =\frac{2 t}{a} \int_{-\infty}^{+\infty} \psi_{0}(k)|k|^{(1-\varepsilon-z)}\left(1-\frac{1-e^{-a|k| z t}}{a|k|^{z} t}\right) d k
\end{aligned}
$$

Applying Proposition B.2, Appendix 2, we conclude that (4.4) is asymptotically Gaussian with variance

$$
2 \frac{\bar{U}^{2} t}{a} \int_{-\infty}^{+\infty} \psi_{0}(k)|k|^{1-\varepsilon-z}\left(1-\frac{1-e^{-a|k| z_{t}}}{a|k|^{z} t}\right) d k
$$


so that the effective equation for $\bar{T}(x, y, t)$ is given by (4.3) with an effective longitudinal diffusivity

$$
D^{*}(t)=\frac{\bar{U}^{2}}{a} \int_{-\infty}^{+\infty} \psi_{0}(k)|k|^{1-\varepsilon-z} e^{-a|k|^{z} t} d k .
$$

As remarked previously, this diffusivity behaves like $D_{\text {III }}^{*(t)}$ for small renormalized times and like $D_{\text {II }}^{*}$ for large renormalized times. For $\varepsilon=\frac{8}{3}$ and $z=\frac{2}{3}$, the Kolmogorov-Obukhov values of the spectral parameters, we obtain the effective diffusivity

$$
D^{*}(t)=\frac{\bar{U}^{2}}{a} \int_{-\infty}^{+\infty} \psi_{0}(k)|k|^{-7 / 3} e^{-a|k|^{2 / 3}} d k
$$

\section{Pair-Distance Distribution and the Fractal Dimension of Interfaces}

The models studied here are extremely rich and reveal in a qualitative fashion many of the observed features of passive advection in turbulent flows, especially when considered in the statistical regimes corresponding to regions II, III (or II ${ }^{w}$, III $^{w}$ ), and on the interfaces between these regions. In this section, we study the following quantities of practical importance associated with higher-order statistics for the passive scalar:

(i) the probability distribution function for the advection of pairs of particles, which is intimately related to the correlation function

$$
\left\langle T_{\delta}(x, y, t) T_{\delta}\left(x^{\prime}, y^{\prime}, t\right)\right\rangle,
$$

(ii) the fractal dimension of advected line elements, or interfaces, evolving in the flow.

In Paragraph V.1 we present the results concerning relative diffusion of pairs of particles and their application to computing the pair-dispersion or pair-diffusivity for Kolmogorov-Obukhov spectra, corresponding to $\varepsilon=8 / 3, z=2 / 3$, as well as the pair-diffusivity corresponding to intermittency corrections to the KolmogorovObukhov theory. In Paragraph V.2 we discuss the results predicted by the models for the fractal dimensions of interfaces in the turbulent simple shear flows and apply the results to the physically relevant regimes corresponding to the neighborhood of the Kolmogorov-Obukhov point in the $(\varepsilon, z)$ phase-diagrams. A rigorous mathematical derivation of these results concerning relative dispersion and the fractal dimensions of interfaces is given in V.3. Finally, in V.4 we make some remarks concerning the invariance of the effective pair-distance distribution under the scaling group associated with the function $\rho=\rho(\delta)$.

V.1. Pair-Distance Distribution. We describe in this section the predictions of the models for relative diffusion of pairs of particles and give a rigorous derivation of the evolution equation for the pair-distance distribution in Kolmogorov-Obukhov turbulence $(\varepsilon=8 / 3, z=2 / 3)$; as well as in the neighboring regimes of the phasediagrams corresponding to intermittency corrections.

We recall first the definition of the pair-distance distribution function $P\left(l_{1}, l_{2}, t\right)$. If the initial data $T_{0}(x, y)$ is interpreted as an initial density or concentration of 
solute particles in the flow, then the joint probability density for a pair of noninteracting advected particles is given by

$$
Q\left(x, y ; x^{\prime}, y^{\prime}, t\right)=T(x, y, t) T\left(x^{\prime}, y^{\prime}, t\right),
$$

where $(x, y)$ and $\left(x^{\prime}, y^{\prime}\right)$ denote the positions of the two particles. The pair-distance probability density for the system can be obtained from $Q\left(x, y, x^{\prime}, y^{\prime}, t\right)$ by introducing the horizontal and vertical separation variables,

$$
l_{1}=x^{\prime}-x, \quad l_{2}=y^{\prime}-y
$$

and integrating over the position of one of the particles, so that

$$
\begin{aligned}
P\left(l_{1}, l_{2}, t\right) & =\int_{-\infty}^{+\infty} \int_{-\infty}^{+\infty} Q\left(x, y ; x+l_{1}, y+l_{2}, t\right) d x d y \\
& =\int_{-\infty}^{+\infty} \int_{-\infty}^{+\infty} T(x, y, t) T\left(x+l_{1}, y+l_{2}, t\right) d x d y .
\end{aligned}
$$

Accordingly, $P\left(l_{1}, l_{2}, t\right) d l_{1} d l_{2}$ denotes the probability that two particles released independently at $t=0$, have horizontal and vertical separations in the intervals $\left(l_{1}, l_{1}+d l_{1}\right),\left(l_{2}, l_{2}+d l_{2}\right)$, respectively. It is easy to show from $(5.2)$ that $P\left(l_{1}, l_{2}, t\right)$ satisfies the integrodifferential equation

$$
\begin{aligned}
& \frac{\partial P\left(l_{1}, l_{2}, t\right)}{\partial t}+\int_{-\infty}^{+\infty} \int_{-\infty}^{+\infty}\left[\mathbf{u}\left(x+l_{1}, y+l_{2}, t\right)-\mathbf{u}(x, y, t)\right] \cdot \nabla_{\left(l_{1}, l_{2}\right)} Q\left(x, y, x+l_{1}, y+l_{2}, t\right) d x d y \\
& \quad=2 D\left[\frac{\partial^{2} P\left(l_{1}, l_{2}, t\right)}{\partial l_{1}^{2}}+\frac{\partial^{2} P\left(l_{1}, l_{2}, t\right)}{\partial l_{2}^{2}}\right] .
\end{aligned}
$$

Our main result for the long-time, large-distance behavior of the pair-distance distribution for the models is a mathematically rigorous derivation of the equation satisfied by the averaged scalar

$$
\bar{P}\left(l_{1}, l_{2}, t\right)=\lim _{\delta \downarrow 0}\left\langle P\left(\frac{l_{1}}{\delta}, \frac{l_{2}}{\delta}, \frac{t}{\rho^{2}(\delta)}\right)\right\rangle .
$$

In this formula, the scaling function $\rho=\rho(\delta)$ is determined according to the different statistical regimes in the two phase-diagrams presented in the Introduction.

Proposition V.1. (i) In the mean-field regions $I$ and $I^{w}$, the effective equation satisfied by $\bar{P}\left(l_{1}, l_{2}, t\right)$ is

$$
\frac{\partial \bar{P}\left(l_{1}, l_{2}, t\right)}{\partial t}=2 D^{*} \frac{\partial^{2} \bar{P}\left(l_{1}, l_{2}, t\right)}{\partial l_{1}^{2}}+2 D \frac{\partial^{2} \bar{P}\left(l_{1}, l_{2}, t\right)}{\partial l_{2}^{2}},
$$

where $D^{*}$ is the effective longitudinal diffusivity for the averaged passive scalar given in (2.18);

(ii) in regions $I I, \dot{I} \bar{I}^{\bar{w}}, \bar{P}\left(l_{1}, l_{2}, t\right)$ satisfies the equation

$$
\frac{\partial \bar{P}\left(l_{1}, l_{2}, t\right)}{\partial t}=D_{\mathrm{II}}^{*}\left(l_{2}\right) \frac{\partial^{2} \bar{P}\left(l_{1}, l_{2}, t\right)}{\partial l_{1}^{2}},
$$


where $D_{\mathrm{II}}^{*}\left(l_{2}\right)$ is the pair-distance effective diffusivity, given by

$$
D_{\text {II }}^{*}\left(l_{2}\right)=\frac{2 \bar{U}^{2}}{a} \int_{-\infty}^{+\infty} \psi_{0}(k)|k|^{1-\varepsilon-z}\left[1-\cos k l_{2}\right] d k .
$$

(iii) In regions $I I I$ and $I I I^{\bar{w}}$, the effective equation for $\bar{P}\left(l_{1}, l_{2}, t\right)$ is

$$
\frac{\partial \bar{P}\left(l_{1}, l_{2}, t\right)}{\partial t}=D_{\mathrm{III}}^{*}\left(l_{2}, t\right) \frac{\partial^{2} \bar{P}\left(l_{1}, l_{2}, t\right)}{\partial l_{1}^{2}}
$$

where $D_{\mathrm{III}}^{*}\left(l_{2}, t\right)$ is a space-and-time-dependent pair-diffusivity, given by

$$
D_{\mathrm{III}}^{*}\left(l_{2}, t\right)=\bar{U}^{2} t \int_{-\infty}^{+\infty} \psi_{0}(k)|k|^{1-\varepsilon}\left[1-\cos k l_{2}\right] d k .
$$

(iv) The effective equation in the intermediate boundary between $I I$ and $I I I$, or $I I^{\bar{w}}$ and $I I I^{\bar{w}}$, is

$$
\frac{\partial \bar{P}\left(l_{1}, l_{2}, t\right)}{\partial t}=D_{\text {int }}^{*}\left(l_{2}, t\right) \frac{\partial^{2} \bar{P}\left(l_{1}, l_{2}, t\right)}{\partial l_{1}^{2}}
$$

with effective diffusivity

$$
D_{\text {int }}^{*}\left(l_{2}, t\right)=\frac{2 \bar{U}^{2}}{a} \int_{-\infty}^{+\infty} \psi_{0}(k) k^{1-\varepsilon} e^{-a|k|^{z} t}\left(1-\cos k l_{2}\right) d k
$$

(v) In region IV of the phase-diagram for $\bar{w}=0$, the effective equation for $\bar{P}\left(l_{1}, l_{2}, t\right)$ is the diffusion equation

$$
\frac{\partial^{2} \bar{P}\left(l_{1}, l_{2}, t\right)}{\partial t}=2 D_{\mathrm{IV}}^{*}(t) \frac{\partial^{2} \bar{P}\left(l_{1}, l_{2}, t\right)}{\partial l_{1}^{2}},
$$

where $D_{\mathrm{IV}}^{*}(t)$ is the effective diffusivity corresponding to the single-particle renormalization problem.

(vi) In region $I V^{\bar{w}}$ of the phase diagram with $\bar{w} \neq 0$, the effective equation for $\bar{P}\left(l_{1}, l_{2}, t\right)$ is

$$
\frac{\partial \bar{P}\left(l_{1}, l_{2}, t\right)}{\partial t}=D_{\mathrm{IV} w}^{*}\left(l_{2}\right) \frac{\partial^{2} \bar{P}\left(l_{1}, l_{2}, t\right)}{\partial l_{1}^{2}}
$$

with effective diffusivity

$$
D_{\mathrm{IV} w}^{*}\left(l_{2}\right)=\frac{2 a \bar{U}^{2}}{\bar{w}^{2}} \int_{-\infty}^{+\infty} \psi_{0}(k)|k|^{-1-\varepsilon+z}\left(1-\cos k l_{2}\right) d k .
$$

(vii) Finally, in region $V$ of the phase-diagram for $\bar{w}=0$, the equation of motion for $\bar{P}\left(l_{1}, l_{2}, t\right)$ is nonlocal, as is the case for the corresponding single-particle distribution. If $P_{0}\left(l_{1}, l_{2}\right)=\int_{-\infty} \int_{-\infty} T_{0}(x, y) T_{0}\left(x+l_{1}, y+l_{2}\right) d x d y$ denotes the initial pair-distance density in the macroscopic spatial variables, we have

$$
\bar{P}\left(l_{1}, l_{2}, t\right)=\int_{-\infty}^{+\infty} \bar{G}\left(l_{1}-l_{1}^{\prime}, t\right) P_{0}\left(l_{1}^{\prime}, l_{2}\right) d l_{1}^{\prime},
$$


where $\bar{G}\left(l_{1}, t\right)$ is the Green's function for the pair-distance evolution. This function and the fundamental solution for one particle motions in region $V$ (cf. (3.59)) are related by the equation

$$
\bar{G}\left(l_{1}, t\right)=\frac{1}{\sqrt{2}} \bar{T}\left(\frac{l_{1}}{\sqrt{2}}, t\right) .
$$

The aforementioned results constitute a complete description of the long-time/ large-distance properties for relative diffusion of pairs of particles in all the statistical regimes. The pair-diffusivities depend on the initial vertical separation, $l_{2}$, in some, but not all, phases of the $(\varepsilon, z)$ plane. Notice that in regions $\mathrm{I}, \mathrm{I}^{\bar{w}}, \mathrm{IV}^{\bar{w}}$ and $\mathrm{V}$, the pair diffusivity is independent of $l_{2}$, and is equal to twice the value of the effective single-particle diffusivity (in the special case of region $\mathrm{V}$ this is translated into Eq. (5.18) for the nonlocal pair diffusivity). These regimes correspond to motions of the pairs of particles which are uncorrelated on the macroscopic time-scales. On the other hand, in regions II, II ${ }^{\bar{w}}$, III, III ${ }^{\bar{w}}$ and IV $^{\bar{w}}$, and the boundaries between $\mathrm{II} / \mathrm{III}$ and $\mathrm{II}^{\tilde{w}} / \mathrm{III}^{\bar{w}}$, the relative pair diffusivity depends on the initial $y$-separation, $l_{2}$. This reflects the fact that the positions of the particles remain strongly correlated on the macroscopic space/time scales, due to long-range spatial correlations in the turbulent velocity field. Direct inspection of the equations for the renormalized pairdiffusivities shows that, for finite $l_{2}$, the pair diffusivities $D^{*}\left(l_{2}\right)$ or $D^{*}\left(l_{2}, t\right)$ are smaller than twice the value of the single-particle diffusivities, and converge to that value as $l_{2} \rightarrow \infty$, so that the correlation effects disappear as the separation, $l_{2}$ tends to infinity.

The behavior of the $l_{2}$-dependent pair-diffusivities for small renormalized times is interesting and can be determined easily by rescaling and expanding (5.9), (5.11), (5.13), (5.16) and small $l_{2}$. This gives the following results:

(i) in regions II, II ${ }^{\bar{w}}$, as well as on the boundaries between II and III, and II ${ }^{\bar{w}}$ and III $^{\bar{w}}$, we have

$$
\left.\begin{array}{l}
D_{\mathrm{II}}^{*}\left(l_{2}\right) \\
D_{\mathrm{int}}^{*}\left(l_{2}, t\right)
\end{array}\right\} \cong c_{\mathrm{III}} l_{2}^{\varepsilon+z-2}, \quad l_{2} \ll 1
$$

with

$$
c_{\mathrm{II}}=\frac{2 \bar{U}^{2}}{a} \int_{-\infty}^{+\infty}|k|^{1-\varepsilon-z}(1-\cos k) d k
$$

(ii) In regions III, III ${ }^{\bar{w}}$, we have

$$
D_{\mathrm{III}}^{*}\left(l_{2}, t\right) \cong c_{\mathrm{III}} t l_{2}^{\varepsilon-2}, \quad l_{2} \ll 1,
$$

where

$$
c_{\mathrm{III}}=\bar{U}^{2} \int_{-\infty}^{+\infty}|k|^{1-\varepsilon}(1-\cos k) d k
$$

(iii) The behavior of the pair-diffusivity in region $I^{\bar{w}}$, is given by

$$
D_{\mathrm{IV}^{\bar{w}}}^{*}\left(l_{2}\right) \cong c_{\mathrm{IV}} l_{2}^{\varepsilon-z},
$$


where

$$
c_{\mathrm{IV}}=\frac{2 \bar{U}^{2} a}{\bar{w}^{2}} \int_{-\infty}^{+\infty}|k|^{1-\varepsilon+z}(1-\cos k) d k .
$$

These results can be applied to obtain a mathematically rigorous derivation of the relative diffusion equation in Kolmogorov-Obukhov turbulence in the context of the model, and to predict the consequences that result from departures from the $k^{-5 / 3}$ law by intermittency corrections. In fact, applying the above results to $\varepsilon=8 / 3$ and $z=2 / 3$ we obtain

$$
D^{*}\left(l_{2}\right) \cong \gamma l_{2}^{4 / 3}, \quad l_{2} \ll 1
$$

with

$$
\gamma=\frac{2 \bar{U}^{2}}{a} \int_{-\infty}^{+\infty}|k|^{-7 / 3}(1-\cos k) d k .
$$

This expression for pair diffusivity in the model is in complete qualitative agreement with Richardson's $1926 l^{4 / 3}$ law for relative diffusion in atmospheric turbulence. It is valid for the Kolmogorov-Obukhov values $\varepsilon=8 / 3, z=2 / 3$ as well and small corrections in the constant $\gamma$ and in the exponent $\varepsilon+z-2$ are obtained, for $\varepsilon, z$ near $\varepsilon=8 / 3, z=2 / 3$ in regions II or II $^{\bar{w}}$. However, the Kolmogorov-Obukhov regime lies on the boundary between regions II and III, and the solution of the renormalization problem indicates that in region III the time decorrelation effects become negligible in the long-time large distance limit, with the velocity field behaving as if it were time-independent. We observe that, the limit as $\varepsilon \rightarrow 8 / 3, z \rightarrow 2 / 3$, with $(\varepsilon, z)$ in region III, of the pair diffusivity $D_{\text {III }}^{*}\left(t, l_{2}\right)$ satisfies

$$
D_{\text {III }}^{*}\left(l_{2}, t\right) \cong \tilde{\gamma} t l_{2}^{2 / 3}, \quad l_{2} \ll 1,
$$

with

$$
\tilde{\gamma}=\bar{U}^{2} \int_{-\infty}^{+\infty}|k|^{-5 / 3}(1-\cos k) d k .
$$

The pair-diffusivity in Eq. (5.27) is consistent with the Richardson scaling $X^{2} \sim T^{3}$, but has a completely different dependence on the separation of the pair of diffusing particles. This result in the model has the same form as the heuristically derived intermittency corrections for pair dispersion proposed by Hentschel and Procaccia [17]; in fact (5.19) and (5.27) exhaust all of the various possibilities which were proposed in [17]. In our model, the difference between (5.25) and (5.27), is due to the discontinuity in the dependence of the macroscopic equations of motion on the large-eddy turnover times, as the statistics $(\varepsilon, z)$ cross the boundary between regions II and III.

We observe also that region III corresponds precisely to turbulent regimes for which Taylor's hypothesis concerning the validity of the infinite correlation time approximation is applicable. Thus our rigorous analysis demonstrates that infinitesimal corrections to the Kolmogorov-Obukhov regime $(\varepsilon=8 / 3, z=2 / 3)$ can yield very different effective equations for relative diffusion, which agree with the classical Richardson $l^{4 / 3}$ law for $(\varepsilon, z)$ in region II or on the boundary II/III, but are completely different in region III, which is the region of validity of the Taylor hypothesis. 
V.2. Fractal Dimension of Interfaces and Advected Line Elements. The geometry of level sets of passive scalars or of advected line (or surface) elements in a homogeneous turbulent flow, can be studied very precisely in the context of our models. This is an important issue in the study of real turbulent flows with a well-developed inertial range, in view of the fact that measurements of geometrical objects, such as interfaces, or level sets, have been made recently with considerable accuracy [31]. The regions of the phase diagrams that are directly relevant for turbulent transport are regions II and III, for $\bar{w}=0$, and II $^{\bar{w}}$, III ${ }^{\bar{w}}$ for $\bar{w} \neq 0$, as well as the boundaries separating these regions, which contain the Kolmogorov-Obukhov regime $\varepsilon=8 / 3, z=2 / 3$. Our analysis of the neighborhood of this point leads to rigorous numerical values for the dimensional defect $2-d$, where $d$ is the fractal dimension of the interface, which are in good agreement with measurements by Lovejoy [27], Meneveau and Sreenivasan [31], and the recently obtained bounds of Constantin [12].

To fix ideas, we consider here the motion of a "line-element" of noninteracting Lagrangian particles, coinciding initially with the $y$-axis. The position of a particle which starts in position $(0, y)$ is given by $(X(y, t), Y(y, t))$, where

$$
X(y, t)=\bar{v} t+\int_{0}^{t} u_{\delta}\left(y+\bar{w} s+\sqrt{2 D} \beta_{2}(y, s), s\right) d s+\sqrt{2 D} \beta_{1}(y, t)
$$

and

$$
Y(y, t)=y+\bar{w} t+\sqrt{2 D} \beta_{2}(y, t) .
$$

Here $\beta_{1}(y, \cdot)$ and $\beta_{2}(y, \cdot)$ are independent Brownian paths for each $y$, and $\beta_{i}(y, \cdot)$, $\beta_{i}\left(y^{\prime}, \cdot\right)$ are independent for $y \neq y^{\prime}$. The position of the particles on the line are correlated through the random velocity field, $u_{\delta}(y, t)$. The joint probability distribution for a collection of $N$ particles starting at positions $\left(0, \bar{y}_{1}\right), \ldots,\left(0, \bar{y}_{N}\right)$ is given by the product

$$
T_{1}\left(x_{1}, y_{1}, t\right) T_{2}\left(x_{2}, y_{2}, t\right) \cdots T_{N}\left(x_{N}, y_{N}, t\right),
$$

where $T_{i}(x, y, t)$ is the solution of the advection-diffusion equation with initial data

$$
T_{i}(x, y, 0)=\delta(x) \delta\left(y-\bar{y}_{i}\right) .
$$

The problem of tracking the position of the line element described by $(X(y, t), Y(y, t))$ relative to coarse-grained space and time scales is equivalent, in statistical regimes where Brownian motion is irrelevant (II, III, IV, II $\left.{ }^{\bar{w}}, I I^{\bar{w}}, I^{\bar{w}}\right)$, to studying the asymptotic behavior of the level sets of the equation

$$
\begin{gathered}
\frac{\partial T_{\delta}(x, y, t)}{\partial t}+\frac{\delta}{\rho(\delta)^{2}}\left[\bar{v}+u_{\delta}\left(\frac{y}{\delta}, \frac{t}{\rho(\delta)^{2}}\right)\right] \frac{\partial T_{\delta}(x, y, t)}{\partial x}+\frac{\delta}{(\rho(\delta))^{2}} \bar{w} \frac{\partial T_{\delta}(x, y, t)}{\partial y} \\
=\frac{\delta^{2}}{(\rho(\delta))^{2}} D\left[\frac{\partial^{2} T_{\delta}(x, y, t)}{\partial x^{2}}+\frac{\partial^{2} T_{\delta}(x, y, t)}{\partial y^{2}}\right]
\end{gathered}
$$

with a Heaviside initial data

$$
T_{\delta}(x, y, 0)=H(x)= \begin{cases}0, & x<0 \\ 1, & x \geqq 0\end{cases}
$$


Due to the anisotropy of the models, it is sufficient to consider only the fluctuations of the line-element in the $x$-direction. To study these fluctuations in the macroscopic space/time scales, we make the change of variables $y \leftrightarrow y / \delta, t \rightarrow t /(\rho(\delta))^{2}$ in (5.29), and define the function

$$
\begin{aligned}
F_{\delta}(y, t) & \doteq \delta X\left(\frac{y}{\delta}, \frac{t}{\rho^{2}}\right)-\frac{\delta}{\rho(\delta)^{2}} \bar{v} t \\
& =\delta \int_{0}^{t / \rho^{2}} u_{\delta}\left(\frac{y}{\delta}+\bar{w} s+\sqrt{2 D} \beta_{2}\left(\frac{y}{\delta}, s\right), s\right) d s+\delta \beta_{1}\left(\frac{y}{\delta}, \frac{t}{(\rho(\delta))^{2}}\right) .
\end{aligned}
$$

Our main results are summarized in

Proposition V.2. In all regions of the phase diagrams except in region $V$, the function $F_{\delta}(y, t)$ converges in distribution, for each fixed $t>0$, to a stationary Gaussian random function of $y, \bar{F}(y, t)$ and for $\varepsilon, z$ in region $V, F_{\delta}(y, t)$ converges in distribution to a non-Gaussian, stationary random function $\bar{F}(y, t)$. In all cases, we have $\langle\bar{F}(y, t)\rangle=0$. The covariance of $\bar{F}(y, t)$ varies according to the values of $\varepsilon, z$, as follows:

(i) In regions $I, I^{\bar{w}}, I V$ and $V, \bar{F}(y, t)$ is $\delta$-correlated for each $t$, i.e.,

$$
\langle\bar{F}(y, t) \bar{F}(y+h, t)\rangle=\delta(h)\left\langle|\bar{F}(y, t)|^{2}\right\rangle,
$$

where the variance $\left\langle|\bar{F}(y, t)|^{2}\right\rangle$ is equal to $2 D^{*} t$ in region $I, I^{\bar{w}}$ and $I V$. Here $D^{*}$ represents the effective diffusivity for the renormalized averaged scalar. In region $V$, the variance is given by

$$
\left\langle|\bar{F}(y, t)|^{2}\right\rangle=\frac{t^{1+\varepsilon / 2}}{(2 D)^{1-\varepsilon / 2}} \int_{0}^{\infty} \alpha d v_{\tilde{\varepsilon}}(\alpha),
$$

where $d v_{\varepsilon}(\alpha)$ is the distribution function entering the definition of the effective Green function (cf. (3.59) and [1]).

(ii) In regions $I I$ and $I I^{\bar{w}}$ the limiting random function $\bar{F}(y, t)$ has covariance

$$
\langle\bar{F}(y, t) \bar{F}(y+h, t)\rangle=\frac{\bar{U}^{2} t}{a} \int_{-\infty}^{+\infty} \psi_{\infty}(k)|k|^{1-\varepsilon-z} \cos (k h) d k .
$$

(iii) In regions $I I I$ and $I I I^{\bar{w}}$, the covariance of $\bar{F}(y, t)$ is given by

$$
\langle\bar{F}(y, t) \bar{F}(y+h, t)\rangle=\frac{\bar{U}^{2} t^{2}}{2} \int_{-\infty}^{+\infty} \psi_{0}(k)|k|^{1-\varepsilon} \cos (k h) d k .
$$

(iv) In the intermediate boundaries between $I I$ and $I I I$, or $I I^{\bar{w}}$ and $I I I^{\bar{w}}$ we have

$$
\langle\bar{F}(y, t) \bar{F}(y+h, t)\rangle=\frac{\bar{U}^{2} t}{a} \int_{-\infty}^{+\infty} \psi_{0}(k)|k|^{1-\varepsilon-z}\left[1-\frac{1-e^{-a|k|^{z} t}}{a|k|^{z} t}\right] \cos (k h) d k .
$$

(v) Finally, in region $I V^{\bar{w}}$, the covariance of $\bar{F}(y, t)$ is

$$
\langle\bar{F}(y, t) \bar{F}(y+h, t)\rangle=\frac{\bar{U}^{2} a}{\bar{w}^{2}} \int_{-\infty}^{+\infty} \psi_{0}(k)|k|^{1-\varepsilon+z} \cos (k h) d k
$$

This proposition implies that, in the regimes for which $\bar{F}(y, t)$ is not deltacorrelated, this function is a self-similar, almost surely Hölder-continuous, stationary 
Gaussian process. The corresponding modulus of continuity can be readily computed by noting that

$$
\left\langle|\bar{F}(y, t)-\bar{F}(y+h, t)|^{2}\right\rangle=2\left[\left\langle|\bar{F}(y, t)|^{2}\right\rangle-\langle\bar{F}(y, t) \bar{F}(y+h, t)\rangle\right] .
$$

We observe that, for $\varepsilon, z$ in regions II, II ${ }^{\bar{w}}$, III, III ${ }^{\bar{w}}, \mathrm{IV}^{\bar{w}}$ and on the boundaries II/III and III $^{\bar{w}} /$ III $^{\bar{w}}$, we have

$$
\left\langle|\bar{F}(y, t)-\bar{F}(y+h, t)|^{2}\right\rangle=\int_{0}^{t} D^{*}(s, h) d s,
$$

where $D^{*}(t, h)$ is the pair-diffusivity for the pair-distance distribution. Therefore, using the results of the previous paragraph on the behavior of $D^{*}(t, h)$ for $h \ll 1$, we obtain

$$
\left\langle|\bar{F}(y, t)-\bar{F}(y+h, t)|^{2}\right\rangle \cong C(t)|h|^{2 H},
$$

where $C(t)$ is a time-dependent function, and the Hölder exponent $H$ is given by

$$
H= \begin{cases}\frac{\varepsilon+z-z}{2}, & \text { in II, II }{ }^{\bar{w}} \text { and the boundaries II/III, II }{ }^{\bar{w}} / \mathrm{III}^{\bar{w}} ; \\ \frac{\varepsilon-2}{2}, & \text { in III, III }{ }^{\bar{w}} \\ \frac{\varepsilon-z}{\bar{w}}, & \text { in } \mathrm{IV}^{\bar{w}} .\end{cases}
$$

By a theorem of S. Orey [34], we identify the Hölder exponent with the dimensional defect $H=2-d$, where $d$ is the fractal dimension of the line element in the longtime, large distance limit, described by the graph of the function $\bar{F}(y, t)$. In this fashion, we have achieved a completely rigorous characterization of the fractal dimension of the typical interface or line element in the context of the special flow fields in the model. This is summarized in Table 2 of the introduction.

We can apply these results to study the neighborhood of the KolmogorovObukhov regime. For $\varepsilon=8 / 3, z=2 / 3$ we obtain

$$
H=\frac{2}{3}, \quad d=\frac{4}{3},
$$

which also agrees with the values in an infinitesimal neighborhood of the Kolmogorov-Obukhov regime in regions II or II $^{\bar{w}}$. However, taking the limit of $H$ as $(\varepsilon, z)$ approach $\left(\frac{8}{3}, \frac{2}{3}\right)$ from regions $I I I$ or $I I I^{\bar{w}}$, we obtain the different results

$$
H=\frac{1}{3}, \quad d=\frac{5}{3} .
$$

Both sets of values were proposed by several authors $[12,17,27,31]$ on the basis of experiments or dimensional analysis. Our analysis of the model, from first principles, shows that discrepancies in fractal dimensions are related to the fact that the Kolmogorov-Obukhov regime lies on the boundary between two different phases in the $(\varepsilon, z)$ plane.

V.3. Proofs of Propositions V.1 and V.2. We present here the proofs of the statements of the previous two paragraphs. We begin by giving a proof of Proposition V.2, concerning the asymptotic behavior of an advected front, and then we show how the results of Proposition V.1 on the pair-distance distribution can be essentially deduced from Proposition V.2. 
V.3.A. Proof of Proposition V.2. We need to show that the function

$$
y \mapsto F_{\delta}(y, t)=\delta \int_{0}^{t / \rho^{2}} u_{\delta}\left(\frac{y}{\delta}+\bar{w} s+\sqrt{2 D} \beta_{2}(y, s), s\right) d s+\delta \beta_{1}\left(\frac{y}{\delta}, \frac{t}{(\rho(\delta))^{2}}\right)
$$

converges in distribution, for each $t>0$, to a stationary random function $\bar{F}(y, t)$, satisfying properties (i) through (v) in the statement of Proposition V.2. To do this, it is sufficient to compute the asymptotic distribution of the random variables $\boldsymbol{\Phi}_{\delta}$, given by

$$
\begin{aligned}
\boldsymbol{\Phi}_{\delta}= & \sum_{j=1}^{N} \lambda_{j} F_{\delta}\left(y_{j}, t\right) \\
= & \delta \int_{0}^{t / \rho^{2}}\left[\sum_{j=1}^{N} \lambda_{j} u_{\delta}\left(\frac{y_{j}}{\delta}+\bar{w} s+\sqrt{2 D} \beta_{2}\left(\frac{y_{j}}{\delta}, s\right), s\right)\right] d s \\
& +\delta \sqrt{2 D} \sum_{j=1}^{N} \lambda_{j} \beta_{1}\left(\frac{y_{j}}{\delta}, \frac{t}{\rho^{2}}\right),
\end{aligned}
$$

where $\left\{y_{1}, \ldots, y_{N}\right\}$ is a set of real numbers and $\left(\lambda_{1}, \ldots, \lambda_{N}\right)$ is an arbitrary vector, and to show that the limiting variance satisfies

$$
\sigma^{2}=\sum_{j, k=1}^{N} \lambda_{j} \lambda_{k}\left\langle\bar{F}\left(y_{j}, t\right) \bar{F}\left(y_{k}, t\right)\right\rangle
$$

where the covariances $\left\langle\bar{F}\left(y_{j}, t\right) \bar{F}\left(y_{k}, t\right)\right\rangle$ are determined by Proposition V.2, according to the different values of $\varepsilon, z$. It is not hard to verify that the variances of $\boldsymbol{\Phi}_{\delta}=\sum_{j} \lambda_{j} F_{\delta}\left(y_{j}, t\right)$ are uniformly bounded in $\delta$. Therefore, in the case of Gaussian velocity fields, the proof of Proposition V.2 reduces essentially to the calculation of the limit of the variances $\left\langle\boldsymbol{\Phi}_{\delta}^{2}\right\rangle$ as $\delta \rightarrow 0$. For non-Gaussian statistics satisfying the assumptions of Models A and B, a suitable central limit theorem can be applied. We shall consider here only the latter cases, given that the analysis of Gaussian fields is straightforward, and can be carried out using the approach of [1].

The proof of Proposition V.2 is divided into two parts. First, we consider regions in which the limiting process is Hölder continuous, i.e. regions II, II ${ }^{\bar{w}}$, III, III ${ }^{\bar{w}}, \mathrm{IV}^{\bar{w}}$ and the boundaries II/III and $\mathrm{II}^{\bar{w}} / \mathrm{III}^{\bar{w}}$, and in a second step, we study the regions for which the limiting process is $\delta$-correlated in $y$, corresponding to regions I, I ${ }^{\bar{w}}$, IV and V.

Step 1. We define the auxiliary vector field

$$
\mathbf{U}_{\delta}(z, s)=\sum_{j=1}^{N} \lambda_{j} u_{\delta}\left(\frac{y_{j}}{\delta}+z, s\right)
$$

and introduce the function

$$
f(k)=\left[\sum_{j=1}^{N} \lambda_{j} \cos \left(y_{j} k\right)\right] \psi_{0}^{1 / 2}(k),
$$

where $\psi_{0}(k)$ is the standard infrared cutoff entering the definition of $E_{\delta}(k, \omega)$ in (3.1). Clearly, the fields $\mathbf{U}_{\delta}(z, s)$ and $u_{\delta}(y, s)$ have a similar structure, except for the fact that $f(k)$ is an oscillating function of $k$. In particular, if $u_{\delta}(y, t)$ satisfies the 
assumptions of Models $\mathrm{A}$ or $\mathrm{B}$, the same is true for $\mathbf{U}_{\delta}$. The spectrum of $U_{\delta}(z, t)$ is given by

$$
\begin{aligned}
\mathbf{E}_{\delta}(k, \omega) & =\frac{\bar{U}^{2}(f(k / \delta))^{2} \psi_{\infty}(k)|k|^{1-\varepsilon-z}}{a \pi\left[1+\omega^{2} / a^{2}|k|^{2 z}\right]} \\
& =\left[\sum_{j=1}^{N} \lambda_{j} \cos \left(y_{j} k / \delta\right)\right]^{2} E_{\delta}(k, \omega),
\end{aligned}
$$

where $E_{\delta}(k, \omega)$ is the power-energy spectrum of $u_{\delta}(y, t)$. In the regions of interest, the effects of Brownian motion are negligible, as shown in Sect. III, and, similarly, Brownian motion can be neglected in the expression for the random variables $\boldsymbol{\Phi}_{\delta}$ in (5.48). Thus $\boldsymbol{\Phi}_{\delta}$ can be replaced by

$$
\delta \int_{0}^{t / \rho^{2}}\left[\sum_{j=1}^{N} \lambda_{j} u_{\delta}\left(\frac{y_{j}}{\delta}+\bar{w} s, s\right)\right] d s=\delta \int_{0}^{t / \rho^{2}} \mathbf{U}_{\delta}(\bar{w} s, s) d s .
$$

Applying the arguments of Sect. III, to the integrals $\delta \int_{0}^{t / \rho^{2}} \mathbf{U}_{\delta}(\bar{w} s, s) d s$, we conclude that, in regions II and $\mathrm{II}^{\bar{w}}, \boldsymbol{\Phi}_{\delta}$ converges to a Gaussian random variable with mean zero and variance

$$
\begin{aligned}
\sigma_{\mathrm{II}}^{2} & =\frac{2 \bar{U}^{2}}{a} \int_{-\infty}^{+\infty}(f(k))^{2}|k|^{1-\varepsilon-z} d k \\
& =\frac{2 \bar{U}^{2}}{a} \int_{-\infty}^{+\infty}\left|\sum_{j=1}^{N} \lambda_{j} e^{i y_{j} k}\right|^{2} \psi_{0}(k)|k|^{1-\varepsilon-z} d k \\
& =\frac{2 \bar{U}^{2}}{a} \sum_{j, k=1}^{N} \lambda_{i} \lambda_{j} \int_{-\infty}^{+\infty} \psi_{0}(k) \cos \left[k\left(y_{j}-y_{k}\right)\right]|k|^{1-\varepsilon-z} d k
\end{aligned}
$$

It follows that the covariance of $\bar{F}(y, t)$ is given by

$$
\left\langle\bar{F}\left(y_{j}, t\right) \bar{F}\left(y_{k}, t\right)\right\rangle=\frac{2 \bar{U}^{2}}{a} \int_{-\infty}^{+\infty} \psi_{0}(k) \cos \left[k\left(y_{j}-y_{k}\right)\right]|k|^{1-\varepsilon-z} d k,
$$

which is what we wanted to show. The same argument applies in regions III, III ${ }^{\bar{w}}$, $\mathrm{IV}^{\bar{w}}$ and in the intermediate segments II/III, II ${ }^{\bar{w}} / \mathrm{III}^{\bar{w}}$. Accordingly, the asymptotic variances of $\boldsymbol{\Phi}_{\delta}$ in such regions are

$$
\begin{aligned}
\sigma_{\mathrm{III}}^{2} & =\bar{U}^{2} t \int_{-\infty}^{+\infty}(f(k))^{2}|k|^{1-\varepsilon} d k \\
& =\bar{U}^{2} t \sum_{j, k=1}^{N} \lambda_{j} \lambda_{k} \int_{-\infty}^{+\infty} \psi_{0}(k) \cos \left[k\left(y_{j}-y_{k}\right)\right]|k|^{1-\varepsilon} d k
\end{aligned}
$$

in regions III, III ${ }^{\bar{w}}$,

$$
\begin{aligned}
\sigma_{\mathrm{IV}}^{2} & =\frac{2 \bar{U}^{2} a t}{\bar{w}^{2}} \int_{-\infty}^{+\infty}(f(k))^{2}|k|^{-1-\varepsilon-z} d k \\
& =\frac{2 \bar{U}^{2} a t}{\bar{w}^{2}} \sum_{j, k=1}^{N} \lambda_{j} \lambda_{k} \int_{-\infty}^{+\infty} \psi_{0}(k) \cos \left[k\left(y_{j}-y_{k}\right)|k|^{1-\varepsilon+z} d k\right.
\end{aligned}
$$


in region $I V^{\bar{w}}$, and

$$
\begin{aligned}
\sigma_{\text {int }}^{2} & =\frac{2 \bar{U}^{2} t}{a} \int_{-\infty}^{+\infty}(f(k))^{2}|k|^{1-\varepsilon-z}\left[1-\frac{1-e^{-a|k|^{2} t}}{a|k|^{z}}\right] d k \\
& =\frac{2 \bar{U}^{2} t}{a} \sum_{j, k=1}^{N} \int_{-\infty}^{+\infty} \psi_{0}(k) \cos k\left(y_{j}-y_{z}\right)|k|^{1-\varepsilon-z}\left[1-\frac{1-e^{-\left.a|k|\right|^{z} t}}{a|k|^{z}}\right] d k
\end{aligned}
$$

on the boundaries II/III and II ${ }^{\bar{w}} / \mathrm{III}^{\bar{w}}$. This concludes the discussion of the regimes with Hölder-continuous fronts.

Step 2. We consider next the regimes for which $\bar{F}(y, t)$ is $\delta$-correlated, i.e. regions I, I ${ }^{\bar{w}}, \mathrm{IV}$, and V.

For velocity fields in regions I and $\mathrm{I}^{\bar{w}}$, the characteristic function of $\boldsymbol{\Phi}_{\delta}$ can be written in the form

$$
\begin{aligned}
E\left\{e^{i \theta \boldsymbol{\Phi}_{\delta}}\right\}= & \exp \left[-D \theta^{2}\left(\sum_{j=1}^{N} \lambda_{j}^{2}\right) t\right] \\
& \cdot\left\langle\prod_{j=1}^{N} E\left\{\exp \left[i \theta \lambda_{j} \delta \int_{0}^{t / \rho^{2}} u_{\delta}\left(\left(y_{j} / \delta\right)+\bar{w} s+\sqrt{2 D} \beta_{2}\left(y_{j} / \delta, s\right), s\right) d s\right]\right\}\right\rangle .
\end{aligned}
$$

We know from the theory of homogenization $[4,7,23,33,36,37]$, that each factor in (5.59),

$$
E\left\{\exp \left[i \theta \lambda_{j} \delta \int_{0}^{t / \rho^{2}} u_{\delta}\left(\left(y_{j} / \delta\right)+\bar{w} s+\sqrt{2 D} \beta_{2}\left(y_{j} / \delta, s\right), s\right) d s\right]\right\}
$$

for $1 \leqq j \leqq N$, converges in probability to its homogenized limit,

$$
e^{-\theta^{2} \lambda_{J}^{2}\left(D^{*}-D\right) t}
$$

where $D^{*}$ is the homogenized longitudinal diffusivity, given in (2.18). [In Appendix 1, we derive a weaker result, concerning the behavior of the average of (5.60) with respect to velocity statistics.] We conclude that

$$
\lim _{\delta \downarrow 0}\left\langle E\left\{e^{i \theta \boldsymbol{\Phi}_{\delta}}\right\}\right\rangle=\exp \left[-D^{*} t \theta^{2}\left(\sum_{j=1}^{N} \lambda_{j}^{2}\right)\right],
$$

so that $\boldsymbol{\Phi}_{\delta}$ converges in distribution to a Gaussian random variable with variance $2 D^{*} t \sum_{j=1}^{N} \lambda_{j}^{2}$, as claimed.

Next, we consider velocity statistics in region IV of the phase diagram for $\bar{w} \neq 0$. In this regime, Brownian motion effects are negligible, so

$$
\boldsymbol{\Phi}_{\delta} \sim \delta \int_{0}^{t / \rho^{2}} \mathbf{U}_{\delta}(0, s) d s
$$

Following the arguments of Sect. III.1.C, with $f(k)$ instead of the infrared cutoff $\psi_{0}^{1 / 2}(k)$, we can show that the integral in (5.63) is asymptotically Gaussian with 
mean zero and variance

$$
\begin{aligned}
\sigma_{\mathrm{IV}}^{2} & =\lim _{\delta \downarrow 0}\left\langle\left[\delta \int_{0}^{t / \rho^{2}} \mathbf{U}_{\delta}(0, s) d s\right]^{2}\right\rangle \\
& =\lim _{\delta \downarrow 0} \frac{2 \bar{U}^{2} t}{a}(a t)^{1+(\varepsilon-2) / z} \int_{-\infty}^{+\infty} f\left(\frac{g(\delta) k}{\delta}\right)^{2}|k|^{1-\varepsilon-z}\left(1-\frac{1-e^{-|k|^{z}}}{|k|^{2}}\right) d k
\end{aligned}
$$

where $g(\delta)=\delta^{2 /(2 z+\varepsilon-2)} \gg \delta$. (See Sect. III.1.C.) In fact, the variable $\delta \int_{0}^{t / \rho^{2}} \mathbf{U}_{\delta}(0, s) d s$ can be written as a sum of blobs, satisfying the assumptions of Proposition B.2 in Appendix 2; see also the Remark following the proof of that proposition. The rapid oscillation of $f\left(\frac{g(\delta) k}{\delta}\right)$ as $\delta \rightarrow 0$ implies that the limit in (5.64) is given by

$$
\begin{aligned}
\sigma_{\mathrm{IV}}^{2} & =\frac{2 \bar{U}^{2} t}{a} \int_{-\infty}^{+\infty}\left(\sum_{j} \lambda_{j}^{2}\right)|k|^{1-\varepsilon-z}\left[1-\frac{1-e^{-|k|^{z}}}{|k|^{z}}\right] d k \\
& =2 D_{\mathrm{IV}}^{*}(t)\left(\sum_{j=1}^{N} \lambda_{j}^{2}\right) .
\end{aligned}
$$

This shows that $\bar{F}(y, t)$ is a Gaussian white noise for $\varepsilon, z$ in region IV.

In the same way, the asymptotic behavior of $F_{\delta}(y, t)$ for $\varepsilon, z$ in region $\mathrm{V}$ can be established by using the method of III.1.D, which consists in applying Proposition B. 2 to the random variables

$$
\delta \int_{0}^{t / \rho^{2}}\left[\sum_{j=1}^{N} \lambda_{j} u_{\delta}\left(\frac{y_{j}}{\delta}+\sqrt{2 D} \beta_{2}\left(\frac{y_{j}}{\delta}, s\right), s\right)\right] d s .
$$

The straightforward details of the proof are omitted. Note that $\bar{F}(y, t)$ is not Gaussian, since the one-particle Green function, given by (3.59) is non-local. The asymptotic variance of the integral in (5.66) can be found by an explicit calculation. It is given by

$$
\sigma_{\mathrm{V}}^{2}=\frac{t^{1+\varepsilon / 2}}{(2 D)^{1-\varepsilon / 2}}\left(\sum_{j=1}^{N} \lambda_{j}^{2}\right) \int_{0}^{+\infty} \alpha d v_{\varepsilon}(\alpha)
$$

where $v_{\varepsilon}(\alpha)$ is defined in Sect. III and [1]. We conclude that $\bar{F}(y, t)$ is a non-Gaussian, $\delta$-correlated random process for $\varepsilon, z$ in region $V$. This concludes the proofs of Proposition V.2.

V.3.B. Proof of Proposition V.1. Consider an initial datum

$$
T_{0}(\delta x, \delta y)=\iint \hat{T}_{0}(\xi, \eta) e^{i \delta \xi x+i \delta \eta y} d \xi d \eta
$$

representing the (unnormalized) distribution of particles at time $t=0$. If $T(x, y, t)$ denotes the corresponding solution of the advection-diffusion equation (2.1), we have

$$
T(x, y, t) T\left(x^{\prime}, y^{\prime}, t^{\prime}\right)=\iiint \int \hat{T}_{0}(\xi, \eta) \hat{T}_{0}\left(\xi^{\prime} \eta^{\prime}\right) A\left(\xi, \eta, \xi^{\prime}, \eta^{\prime}, x, y, x^{\prime}, y^{\prime}, t\right) \delta \xi d \eta d \xi^{\prime} d \eta^{\prime},
$$


where

$$
\begin{aligned}
A\left(\xi, \eta, \xi^{\prime}, \eta^{\prime}, x, y, x^{\prime}, y^{\prime}, t\right)= & e^{i \delta \xi x+i \delta \eta y+i \delta \xi^{\prime} x^{\prime}+i \delta \eta^{\prime} y^{\prime}+i \delta\left(\xi+\xi^{\prime}\right) \bar{w} t+i \delta\left(\eta+\eta^{\prime}\right) \bar{w} t-\delta D t \xi^{2}} \\
& \cdot e^{-\delta D^{*}\left(\xi^{\prime}\right)^{2} t} \times E\left\{e^{i B\left(\xi, \eta, \xi^{\prime}, \eta^{\prime}, y, y^{\prime}, t\right)}\right\}
\end{aligned}
$$

with

$$
\begin{aligned}
& B\left(\xi, \eta, \xi^{\prime}, \eta^{\prime}, y, y^{\prime}, t\right) \\
& =\delta \eta \sqrt{2 D} \beta_{2}(t)+\delta \eta^{\prime} \sqrt{2 D} \beta_{2}^{\prime}(t)+\delta \xi \int_{0}^{t} u_{\delta}\left(y+\sqrt{2 D} \beta_{2}(s)+\bar{w} s, s\right) d s \\
& \quad+\delta \xi^{\prime} \int_{0}^{t} u_{\delta}\left(y^{\prime}+\sqrt{2 D} \beta_{2}^{\prime}(s)+\bar{w} s, s\right) d s .
\end{aligned}
$$

Here $\beta_{2}(s)$ and $\beta_{2}^{\prime}(s)$ are independent Brownian motions. Averaging

$$
A\left(\xi, \eta, \xi^{\prime}, \eta^{\prime}, x, y, x^{\prime}, y^{\prime}, t\right)
$$

with respect to velocity statistics and using the homogeneity of $U_{\delta}(y, t)$ we obtain $\left\langle A\left(\xi, \eta, \xi^{\prime}, \eta^{\prime}, x, y, x^{\prime}, y^{\prime}, t\right\rangle=e^{i \delta \xi x+i \delta \eta y+i \delta \xi^{\prime} x^{\prime}+i \delta \eta^{\prime} y^{\prime}-\delta^{2} D t \xi^{2}-\delta^{2} D t\left(\xi^{\prime}\right)^{2}}\right.$

$$
\cdot e^{i \delta\left(\xi+\xi^{\prime}\right) \bar{v} t+i \delta\left(\eta+\eta^{\prime}\right) \bar{w} t} \times\left\langle E\left\{e^{i B\left(\xi, \eta, \xi^{\prime}, \eta^{\prime}, 0, y^{\prime}-y, t\right.}\right\}\right\rangle .
$$

Recalling that $P\left(l_{1}, l_{2}, t\right)$ is the spatial convolution of $T(x, y, t)$ with itself (cf. (5.4)), we have

$$
\left\langle P\left(l_{1}, l_{2}, t\right)\right\rangle=\iint\left|\hat{T}_{0}(\xi, \eta)\right|^{2}\left\langle A\left(\xi, \eta,-\xi,-\eta, 0,0, l_{1}, l_{2}, t\right)\right\rangle d \xi d \eta
$$

Therefore, the asymptotic behavior of $\left\langle P\left(\frac{l_{1}}{\delta}, \frac{l_{2}}{\delta}, \frac{t}{\rho^{2}}\right)\right\rangle$ is determined by the limit as $\delta \rightarrow 0$ of the quantity

$$
\begin{aligned}
& \left\langle A\left(\xi, \eta,-\xi,-\eta, 0,0, \frac{l_{1}}{\delta}, \frac{l_{2}}{\delta}, \frac{t}{\rho^{2}}\right)\right\rangle \\
& =e^{-i \xi l_{1}-i \eta l_{2}-\left(2 \delta^{2} / \rho^{2}\right) D t \xi^{2}} \times\left\langle E\left\{e^{i B\left(\xi, \eta,-\xi,-\eta, 0,\left(l_{2} / \delta\right),\left(t / \rho^{2}\right)\right)}\right\}\right\rangle .
\end{aligned}
$$

In this formula, the exponent inside the Brownian expectation is given by

$$
\begin{aligned}
B\left(\xi, \eta,-\xi,-\eta, 0, \frac{l_{2}}{\delta}, \frac{t}{\rho^{2}}\right)= & \delta \eta \sqrt{2 D} \beta_{2}\left(\frac{t}{\rho^{2}}\right)-\delta \eta \sqrt{2 D} \beta_{2}^{\prime}\left(\frac{t}{\rho^{2}}\right) \\
& +\delta \xi \int_{0}^{t / \rho^{2}} u_{\delta}\left(\sqrt{2 D} \beta_{2}(s)+\bar{w} s, s\right) d s \\
& -\delta \xi^{\prime} \int_{0}^{t / \rho^{2}} u_{\delta}\left(\frac{l_{2}}{\delta}+\sqrt{2 D} \beta_{2}^{\prime}(s)+\bar{w} s, s\right) d s .
\end{aligned}
$$

The proof of Proposition V.1 can be carried out by computing the asymptotic distribution of the quantity $B\left(\xi, \eta,-\xi,-\eta, 0, \frac{l_{2}}{\delta}, \frac{t}{\rho^{2}}\right)$ as $\delta \rightarrow 0$. This is done by setting $\rho=\rho(\delta)$, where $\rho(\delta)$ depends on the specific region under consideration, and applying the appropriate Central Limit Theorem in each case. Notice that in 
the superdiffusive regions, the contributions from the first two summands on the right-hand side of (5.75) are negligible, so that

$$
\begin{aligned}
B\left(\xi, \eta,-\xi,-\eta, 0, \frac{l_{2}}{\delta}, \frac{t}{\rho^{2}}\right) & \sim \delta \xi \int_{0}^{t / \rho^{2}} u_{\delta}\left(\sqrt{2 D} \beta_{2}(s)+\bar{w} s, s\right) d s \\
& -\delta \xi \int_{0}^{t / \rho^{2}} u_{\delta}\left(\frac{l_{2}}{\delta}+\sqrt{2 D} \beta_{2}^{\prime}(s)+\bar{w} s, s\right) d s \\
& \sim \boldsymbol{\Phi}_{\delta}(t),
\end{aligned}
$$

(cf. (5.48)), with $N=2, y_{1}=0, y_{2}=l_{2}, \lambda_{1}=\xi$ and $\lambda_{2}=-\xi$. Thus, in such cases we have

$$
\left\langle P\left(\frac{l_{1}}{\delta}, \frac{l_{2}}{\delta}, \frac{t}{\rho^{2}}\right)\right\rangle \sim \iint\left|\hat{T}_{0}(\xi, \eta)\right|^{2} e^{-i \xi l_{1}-i \xi l_{2}}\left\langle E\left\{e^{i \Phi_{\delta}(t)}\right\}\right\rangle,
$$

and the effective equation satisfied by $\bar{P}\left(l_{1}, l_{2}, t\right)=\lim _{\delta \downarrow 0}\left\langle P\left(\frac{l_{1}}{\delta}, \frac{l_{2}}{\delta}, \frac{t}{\rho^{2}}\right)\right\rangle$ can be deduced immediately from Proposition V.2, which characterizes the asymptotic distribution of $\boldsymbol{\Phi}_{\delta}$. Finally, the evolution of the pair distance distribution in the mean-field regimes is determined by noticing that, for $\varepsilon, z$ in regions $I$ and $I^{\bar{w}}$, we have

$$
\begin{aligned}
& \lim _{\delta \downarrow 0}\left\langle T_{\delta}\left(\frac{x}{\delta}+\frac{\bar{v} t}{\rho^{2}}, \frac{y}{\delta}+\frac{\bar{w} t}{\rho^{2}}, \frac{t}{\rho^{2}}\right) T_{\delta}\left(\frac{x^{\prime}}{\delta}+\frac{\bar{v} t}{\rho^{2}}, \frac{y^{\prime}}{\delta}+\frac{\bar{w} t}{\rho^{2}}, \frac{t}{\rho^{2}}\right)\right\rangle \\
& =\bar{T}(x, y, t) \bar{T}\left(x^{\prime}, y^{\prime}, t^{\prime}\right)
\end{aligned}
$$

so that $\bar{P}\left(l_{1}, l_{2}, t\right)$ satisfies, from (5.4)

$$
\bar{P}\left(l_{1}, l_{2}, t\right)=\iint\left|\hat{T}_{0}(\xi, \eta)\right|^{2} e^{i \xi l_{1}+i \eta l_{1}-2 D^{*} t \xi^{2}-2 D t \eta^{2}} d \xi d \eta .
$$

This concludes the proof of Proposition V.1.

V.4. Invariance of the Renormalized Equations under the Scaling Group $\left(l_{1}, l_{2}, t\right) \rightarrow$ $\left(\frac{l_{1}}{\delta}, \frac{l_{2}}{\delta}, \frac{t}{\rho^{2}}\right)$. In the previous sections of this paper, we have derived limit equations for the renormalized ensemble averaged mean, $\bar{T}(x, y, t)$, and the renormalized pairdistance distribution function $\bar{P}(x, y, t)$. These renormalized equations are derived under the large-scale renormalized transformation,

$$
(x, y, t) \mapsto\left(\frac{x}{\delta}, \frac{y}{\delta}, \frac{t}{\rho(\delta)^{2}}\right)
$$

so it is natural to investigate whether the effective equations for $\bar{T}(x, y, t)$ and $P\left(l_{1}, l_{2}, t\right)$ are invariant under the scaling group,

$$
\left(l_{1}, l_{2}, t\right) \mapsto\left(\frac{l_{1}}{\delta}, \frac{l_{2}}{\delta}, \frac{t}{\rho(\delta)^{2}}\right) .
$$

It is convenient to refer to Tables 1 and 2 of the introduction for the remainder of the discussion. 
In regions, $\mathrm{I}, \mathrm{I}^{\bar{w}}, \mathrm{IV}$, and $\mathrm{V}$, the equations for $T$ and $P$ are automatically invariant under the scaling group of transformations in (5.80). For the regions I, $\mathrm{I}^{\bar{w}}$, and IV, this is a straightforward calculation which utilizes the specific time dependent form for the diffusion coefficient in region IV. The scale invariance for the nonlocal equation in region $\mathrm{V}$ requires a slightly more subtle argument. For any number $\alpha>0$, the diffusion equation

$$
\frac{\partial \bar{T}}{\partial t}=t^{\varepsilon / 2} \alpha \bar{T}_{x x}
$$

is invariant under the scaling transformation appropriate for region $\mathrm{V}$ given by $\rho(\lambda)=\lambda^{1 /(1+\varepsilon / 2)}$ so that the same is true for the corresponding Green's function, $K(x, t, \alpha)$. Since the actual Green's function is region $\mathrm{V}$ is given by the superposition.

$$
K^{\varepsilon}(x, t)=\int_{0^{+}}^{\infty} K(x, t, \alpha) d v_{\varepsilon}(\alpha)
$$

with an appropriate probability measure $v_{\varepsilon}$ (see (1.10)-(1.12) and Sect. 4 of [1]), it follows that $K^{\varepsilon}(x, t)$ is also invariant for the appropriate symmetry group from region $\mathrm{V}$. Thus, we have established the scale invariance of the renormalized theory for regions $I, I^{\bar{w}}, I V$, and $V$, and also for appropriate steady velocity fields at $z=+\infty$ in regions $\mathrm{I}, \mathrm{I}^{\bar{w}}$, and $\mathrm{V}$. The scale invariance in these regions is a reflection of the fact that the infrared cutoff $\psi_{0}(|k|)$ does not enter into the renormalized diffusivity in these regions.

However, the effective equations for $\bar{T}(x, y, t)$ in regions II, II ${ }^{\bar{w}}, \mathrm{III}, \mathrm{III}^{\bar{w}}, \mathrm{IV}^{\bar{w}}$, and on the boundaries II/III and II ${ }^{\bar{w}} /$ III $^{\bar{w}}$ are not invariant under the corresponding scaling symmetry group. This peculiar fact is related to the fact (summarized in Table 1) that the coefficient of diffusion for the renormalized equation for these regions depends upon the infrared cutoff $\psi_{0}(|k|)$. This apparent lack of invariance reflects the fact that the coarse-gained motion of fronts and pairs of particles is correlated in these regions and hence the averaged scalar $\bar{T}(x, y, t)$, which can be interpreted as a marginal probability distribution for the evolution of a single particle, does not describe completely the macroscopic evolution of the system. Nevertheless, there is a more subtle fashion in which the renormalized equations in these regions reflect the scale invariance in (5.80). This can be seen through the renormalized pair-distance function $P\left(l_{1}, l_{2}, t\right)$. For the pair-distance distribution function $\bar{P}\left(l_{1}, l_{2}, t\right)$, one can easily verify that the equation satisfied by $\bar{P}\left(l_{1}, l_{2}, t\right)$ is invariant under the transformation $\left(l_{1}, l_{2}, t\right) \rightarrow\left(\frac{l_{1}}{\delta}, \frac{l_{2}}{\delta}, \frac{t}{\rho^{2}}\right)$, in the limit $\left|l_{2}\right| \ll 1$, obtained by substituting in the evolution equations for $\bar{P}\left(l_{1}, l_{2}, t\right)$ the expressions for the pair-diffusivity $D^{*}\left(l_{2}, t\right)$ for $l_{1} \ll 1$ given in (5.19)-(5.24). To emphasize this point, we consider the pair diffusion equation in region II with the expansion from (5.19) for $\left|l_{2}\right| \ll 1$ and obtain,

$$
\frac{\partial P\left(l_{1}, l_{2}, t\right)}{\partial t}=c_{\mathrm{II}} l_{2}^{\varepsilon+z-2} \frac{\partial^{2} P}{\partial l_{1}^{2}}
$$

With the scaling group appropriate for region II for (5.80), i.e., with $\rho(\lambda)=\lambda^{(4-\varepsilon-z) / 2}$, the equation in (5.81) is scale invariant. Similar calculations apply to the other regions of renormalization. Thus, the equation for the pair distance distribution 
is scale invariant under the symmetry group in (5.80) in the regime, $\left|l_{2}\right| \ll 1$ but the equation for $\bar{T}$, involving the large distance limit $l_{2} \rightarrow \infty$, is not scale invariant.

\section{Appendix 1. The Mean-Field Regions $I$ and $I^{\bar{w}}$}

For values of the parameters $(\varepsilon, z)$ satisfying $\varepsilon+2 z<2+\min (2, z)$ in the case $\bar{w}=0$ (region I), or $\varepsilon<2+\min (2, z)-2 \min (1, z)$ for $\bar{w} \neq 0$ (region $\mathrm{I}^{\bar{w}}$ ) the appropriate time-scaling function is

$$
\rho(\delta)=\delta,
$$

corresponding to Fick's law of normal or mean-field diffusion, $x^{2} \sim t$, and the effective equation satisfied by the average scalar $\bar{T}(x, y, t)=\lim _{\delta \downarrow 0}\left\langle T^{\prime}\left(\frac{x}{\delta}, \frac{y}{\delta}, \frac{t}{\delta^{2}}\right)\right\rangle$ is a parabolic diffusion equation with a renormalized diffusion coefficient in the $x$ direction given by

$$
D^{*}=D+2 \bar{U}^{2} \int_{-\infty}^{+\infty} \frac{\left(D|k|^{2}+a|k|^{z}\right)|k|^{1-\varepsilon} \psi_{\infty}(k) d k}{\left[D|k|^{2}+a|k|^{2}\right]^{2}+\bar{w}^{2}|k|^{2}} .
$$

According to the arguments in Sect. 2.1, this result follows if we establish

Proposition A.1. The random variable

$$
Z_{\delta}(t)=\eta \sqrt{2 D} \beta_{2}(t)+\frac{\xi}{\delta} \int_{0}^{t} u_{\delta}\left(\frac{y+\sqrt{2 D} \beta_{2}(s)}{\delta}+\frac{\bar{w} s}{\delta^{2}}, \frac{s}{\delta^{2}}\right) d s
$$

converges in distribution to a Gaussian random variable with mean zero and variance $2 D \eta^{2}+2\left(D^{*}-D\right) \xi^{2}$.

Proof. To prove this result, we use the homogenization techniques developed by Papanicolaou and Varadhan [35], Kozlov [21] and others [31, 34, 4] to average partial differential equations with random, rapidly oscillating coefficients. For simplicity, we consider only the case $y=0$ in (A.3).

Let us make the change of variables $t=\frac{t^{\prime}}{\delta^{2}}$ in (A.3) so that

$$
Z_{\delta}(t)=\delta \eta \sqrt{2 D} \widetilde{\beta}_{2}\left(\frac{t^{\prime}}{\delta^{2}}\right)+\delta \xi \int_{0}^{t^{\prime} / \delta^{2}} u_{\delta}\left(\bar{w} s+\sqrt{2 D} \widetilde{\beta}_{2}(s), s\right) d s
$$

where $\tilde{\beta}_{2}(\cdot)$ is an auxiliary Brownian motion. Define the auxiliary function, or corrector,

$$
\chi_{\delta}(y, t)=\iint \frac{\left(e^{i k y+i \omega t}-1\right)}{i(\omega+\bar{w} k)+D|k|^{2}} d \hat{u}_{\delta}(k, \omega),
$$

where $d \hat{u}_{\delta}(k, \omega)$ is the spectral measure associated with the random field $u_{\delta}(y, s)$. By construction, $\chi_{\delta}(y, t)$ satisfies

$$
\left(\frac{\partial}{\partial t}+\bar{w} \frac{\partial}{\partial y}+D \frac{\partial^{2}}{\partial y^{2}}\right) \chi_{\delta}(y, t)=u_{\delta}(y, t)
$$


and $\chi_{\delta}(0,0)=0$. By Ito's Formula [29], we have

$$
\begin{aligned}
\chi_{\delta}\left(\bar{w} t+\sqrt{2 D} \tilde{\beta}_{2}(t), t\right)= & \int_{0}^{t} \sqrt{2 D} \frac{\partial \chi_{\delta}}{\partial y}\left(\bar{w} s+\sqrt{2 D} \tilde{\beta}_{2}(s), s\right) d \tilde{\beta}_{2}(s) \\
& +\int_{0}^{t} u_{\delta}\left(\bar{w} s+\sqrt{2 D} \tilde{\beta}_{2}(s), s\right) d s .
\end{aligned}
$$

Hence, for all $\xi, \eta$, we obtain a decomposition of $Z_{\delta}(t)$ as a sum of a stochastic integral and a remainder:

$$
\begin{aligned}
\delta \eta & \sqrt{2 D} \tilde{\beta}_{2}\left(\frac{t}{\delta^{2}}\right)+\delta \xi \int_{0}^{t / \delta^{2}} u_{\delta}\left(\bar{w} s+\sqrt{2 D} \widetilde{\beta}_{2}(s), s\right) d s \\
= & \delta \int_{0}^{t / \rho^{2}}\left[\eta+\xi \frac{\partial \chi_{\delta}}{\partial y}\left(\bar{w} s+\sqrt{2 D} \tilde{\beta}_{2}(s), s\right)\right] \sqrt{2 D} d \tilde{\beta}_{2}(s) \\
& +\xi \delta \chi_{\delta}\left(\frac{\bar{w} t}{\delta^{2}}+\sqrt{2 D} \tilde{\beta}_{2}\left(\frac{t}{\delta^{2}}\right), \frac{t}{\delta^{2}}\right) \\
= & M_{\delta}(t)+R_{\delta}(t) .
\end{aligned}
$$

The proof of Proposition 1 proceeds in two steps:

Step 1. We show that $M_{\delta}(t)$ converges in distribution to a Gaussian random variable with variance $2 D \eta^{2}+2\left(D^{*}-D\right) \xi^{2}$; and

Step 2. We show that $R_{\delta}(t)$ converges to zero in probability.

Proof of Step 1. The variance of the stochastic integral $M_{\delta}(t)$ is

$$
\begin{aligned}
\left\langle E\left\{\left(M_{\delta}(t)\right)^{2}\right\}\right\rangle & =2 \delta^{2} D \int_{0}^{t / \delta^{2}}\left\langle E\left\{\left|\eta+\xi \frac{\partial \chi_{\delta}}{\partial y}\left(\bar{w} s+\sqrt{2 D} \tilde{\beta}_{2}(s), s\right)\right|^{2}\right\}\right\rangle d s \\
& =2 \delta^{2} D \int_{0}^{t / \delta^{2}}\left[\eta^{2}+\xi^{2}\left\langle\left|\frac{\partial \chi_{\delta}}{\partial y}(0,0)\right|^{2}\right\rangle\right] d s \\
& =2 D t\left[\eta^{2}+\xi^{2}\left\langle\left|\frac{\partial \chi_{\delta}}{\partial y}(0,0)\right|^{2}\right\rangle\right]
\end{aligned}
$$

where we used that $\frac{\partial \chi_{\delta}}{\partial y}(y, s)$ is a stationary process with mean zero. In fact, we have

$$
\frac{\partial \chi_{\delta}(y, t)}{\partial y}=\iint \frac{i k e^{i k y+i \omega t}}{i(\omega+\bar{w} k)+D|k|^{2}} d \hat{u}_{\delta}(k, \omega),
$$

so that $\left\langle\frac{\partial \chi_{\delta}(y, t)}{\partial y}\right\rangle=0$, and moreover the variance of $\frac{\partial \chi_{\delta}(y, t)}{\partial y}$ is

$$
\begin{aligned}
\left\langle\left|\frac{\partial \chi_{\delta}(y, t)}{\partial y}\right|^{2}\right\rangle & =\left\langle\left|\iint \frac{i k d \hat{u}_{\delta}(k, \omega)}{i(\omega+\bar{w} k)+D|k|^{2}}\right|^{2}\right\rangle \\
& =\iint \frac{|k|^{2}}{|\omega+\bar{w} k|^{2}+D|k|^{4}} E_{\delta}(k, \omega) d k d \omega
\end{aligned}
$$




$$
\doteq \frac{\bar{U}^{2}}{\pi} \iint \frac{k^{2}}{|\omega+\bar{w} k|^{2}+D^{2}|k|^{4}} \cdot \frac{a^{-1}|k|^{z}}{\left(1+\frac{\omega^{2}}{a^{2}|k|^{2 z}}\right)}|k|^{1-\varepsilon} \psi_{0}\left(\frac{k}{\delta}\right) \psi_{\infty}(k) d \omega d k .
$$

Using the formula

$$
\frac{1}{\pi} \int_{-\infty}^{+\infty} \frac{a^{-1} d \omega e^{i \omega t}}{\left(1+\frac{\omega^{2}}{a^{2}}\right)}=e^{-a|t|}
$$

and Plancherel's identity, we conclude that, for fixed $k \in \mathbf{R}$, the $d \omega$ integral in (A.11) is given by

$$
\begin{aligned}
& \frac{1}{\pi} \int_{-\infty}^{+\infty} \frac{k^{2}}{|\omega+k \bar{w}|^{2}+D^{2}|k|^{2}} \cdot \frac{1}{\left(1+\frac{\omega^{2}}{a|k|^{2 z}}\right)} \frac{1}{a|k|^{z}} d \omega \\
& \quad=\frac{2}{D} \int_{-\infty}^{+\infty} e^{-\left(D|k|^{2}+a|k|^{z}+i \bar{w} k\right)|t|} d t \\
& \quad=\frac{2}{D} \frac{D|k|^{2}+a|k|^{z}}{\left[D|k|^{2}+a|k|^{z}\right]^{2}+\bar{w}^{2}|k|^{2}} .
\end{aligned}
$$

Thus,

$$
\left\langle\left|\frac{\partial \chi_{\delta}(y, t)}{\partial y}\right|^{2}\right\rangle=\frac{2 \bar{U}^{2}}{D} \int_{-\infty}^{+\infty} \psi_{0}\left(\frac{k}{\delta}\right) \psi_{\infty}(k) \frac{\left(D|k|^{2}+a|k|^{z}\right)|k|^{1-\varepsilon} d k}{\left[D|k|^{2}+a|k|^{z}\right]^{2}+\bar{w}^{2}|k|^{2}}
$$

The singularity of the integrand for $|k| \ll 1$ is $|k|^{1-\varepsilon+\min (2, z)-2 \min (1, z)}$ for $\bar{w} \neq 0$ and $|k|^{1-\varepsilon-2 z+\min 2, z}$ for $\bar{w}=0$, so that the integrand is uniformly integrable as $\delta \rightarrow 0$ if and only if $(\varepsilon, z)$ is in region $\mathrm{I}^{\bar{w}}(\bar{w} \neq 0)$ or region I if $\bar{w}=0$. Using (A.9) and (A.14), we obtain

$$
\lim _{\delta \downarrow}\left\langle E\left\{\left(M_{\delta}(t)\right)^{2}\right\}\right\rangle=\left[2 D \eta^{2}+2\left(D^{*}-D\right) \xi^{2}\right] t .
$$

A standard central limit theorem for stochastic integrals [14] which was used by us in [1], allows us to conclude from (A.15) that the stochastic integral $M_{\delta}(t)$ converges in distribution to a Gaussian random variable with mean zero variance $2 D \eta^{2} t+2\left(D^{*}-D\right) \xi^{2} t$, as desired.

Step 2. From (A.8), the remainder term $R_{\delta}(t)$ is given by

$$
\begin{aligned}
R_{\delta}(t) & =\delta \chi_{\delta}\left(\frac{\bar{w} t}{\delta^{2}}+\sqrt{2 D} \widetilde{\beta}_{2}\left(\frac{t}{\delta^{2}}\right), t\right) \\
& =\delta \iint \frac{\left[\exp \left[i(k \bar{w}+\omega) t / \delta^{2}+i \sqrt{2 D} \widetilde{\beta}_{3}\left(\frac{t}{\delta^{2}}\right)\right]-1\right]}{i(\omega+k \bar{w})+D|k|^{2}} d \hat{u}(k, \omega) .
\end{aligned}
$$


Therefore,

$$
\begin{aligned}
& \left\langle E\left\{\left|R_{\delta}(t)\right|^{2}\right\}\right\rangle \\
& =\delta^{2} E\left\{\iint \frac{\left|\exp \left[i(k \bar{w}+\omega) t / \delta^{2}+i k \sqrt{2 D} \tilde{\beta}_{2}\left(\frac{t}{\delta^{2}}\right)\right]-1\right|^{2}}{|\bar{w} k+\omega|^{2}+D^{2}|k|^{4}}\left\langle\left|d \hat{u}_{\delta}(k, \omega)\right|^{2}\right\rangle\right\} \\
& =2 \delta^{2} \iint \frac{\operatorname{Re}\left(1-e^{i(\omega+\bar{w} k) t / \delta^{2}-D k^{2} t / \delta^{2}}\right)}{|\bar{w} k+\omega|^{2}+D^{2}|k|^{4}}\left\langle\left|d \hat{u}_{\delta}(k, \omega)\right|^{2}\right\rangle .
\end{aligned}
$$

We split the domain of integration in two parts, i.e.,

$$
\begin{aligned}
\left\langle E\left\{R_{\delta}^{2}(t)\right\}\right\rangle= & 2 \delta^{2} \int_{|k| \geqq k_{0}}[\cdots]\left\langle\left|d \hat{u}_{\delta}(k, \omega)\right|^{2},\right. \\
& +2 \delta^{2} \int_{|k| \leqq k_{0}}[\cdots]\left\langle\left|d \hat{u}_{\delta}(k, \omega)\right|^{2}\right\rangle .
\end{aligned}
$$

The first term on the right-hand side of (A.18) satisfies

$$
\begin{aligned}
& 2 \delta^{2} \iint_{|k| \leqq k_{0}} \frac{\operatorname{Re}\left(1-e^{i(\omega+\bar{w} k) t / \delta^{2}-D k^{2} t / \delta^{2}}\right)}{D^{2}|k|^{4}+|\omega+\bar{w} k|^{2}}\left\langle\left|d \hat{u}_{\delta}(k, \omega)\right|^{2}\right\rangle \\
& \quad \leqq \frac{2 \delta^{2}}{k_{0}^{2}} \iint \frac{k^{2}}{D^{2}|k|^{4}+|\omega+\bar{w} k|^{2}}\left\langle\left|d \hat{u}_{\delta}(k, \omega)\right|^{2}\right\rangle \\
& \quad \leqq \frac{2 \delta^{2}}{k_{0}^{2}} \int_{-\infty}^{+\infty} \psi_{\infty}(k) \frac{\left(D|k|^{2}+a|k|^{z}\right)|k|^{1-\varepsilon} d k}{\left[D|k|^{2}+a|k|^{2}\right]^{2}+\bar{w}^{2}|k|^{2}} \\
& \quad=0\left(\delta^{2}\right), \text { as } \delta \rightarrow 0 .
\end{aligned}
$$

On the other hand, the second term in (A.18), satisfies

$$
\begin{aligned}
& 2 \delta^{2} \int_{|k| \leqq k_{0}} \frac{\operatorname{Re}\left(1-e^{i(k \bar{w}+\omega) t / \delta^{2}-D k^{2} t / \delta^{2}}\right)}{|\bar{w} k+\omega|^{2}+D^{2}|k|^{4}}\left\langle\mid d \hat{u}_{\delta}(k, \omega)^{2}\right\rangle \\
& \quad \leqq 2 \iint_{|k| \leqq k_{0}} \frac{|k|^{2}}{|\bar{w} k+\omega|^{2}+D^{2}|k|^{4}}\left\langle\left|d \hat{u}_{\delta}(k, \omega)\right|^{2}\right\rangle \\
& \quad \leqq 2 \int_{|k| \leqq k_{0}} \frac{\left(D|k|^{2}+a|k|^{z}\right)|k|^{1-\varepsilon} \psi_{\infty}(k) d k}{\left[D|k|^{2}+a|k|^{2}\right]^{2}+\bar{w}^{2}|k|^{2}} .
\end{aligned}
$$

This final term can be made arbitrarily small by a suitable choice of $k_{0}$. Therefore, combining these two estimates, we conclude that $\lim \left\langle E\left\{R_{\delta}(t)^{2}\right\}\right\rangle=0$, as desired. This completes the proof of Step 2, and hence Proposition A.1 is established.

\section{Appendix 2. Auxiliary Central Limit Theorems for Models A and B}

This appendix contains the statements and proofs of the central limit theorems used in Sect. III, IV and V to compute the effective equations of motion for nonGaussian velocity statistics. The main results presented here can be described as 
central limit theorems for stochastic processes which are "functions of mixing processes," in the sense of Ibragimov [16], cf. also Billingley [6]. the direct application of such general theorems to Models A and B would require, in practice, the verification of appropriate "mixing conditions" and a-priori estimates on the random processes $V_{\delta}(s)$, uniformly in the small parameter $\delta$. While it is likely that such methods may apply to Models A and B, we prefer instead to present here selfcontained proofs of the Central Limit theorems for Models A and B, using only elementary results in Probability Theory. We note that certain velocity fields formed by superpositions of "Poisson blobs," different than the ones studied here, were shown to satisfy suitable mixing conditions by Kesten and Papanicolaou [17]:

Recall that $\left\{U_{n}\right\},\left\{\left(p^{(n)}, \tau^{(n)}\right\}\right.$ which denote respectively a sequence of random variables and a point process in $\mathbf{R}^{2}$, are said to satisfy the hypothesis of Model A if the $\left\{U_{n}\right\}$ are independent, identically distributed random-variables with mean zero and variance $\left\langle U_{n}^{2}\right\rangle=\bar{U}^{2}$ and $\left(p^{(n)}, \tau^{(n)}\right)$ is a stationary, ergodic point process in $\mathbf{R}^{2}$, such that

$$
\lim _{L_{1}, L_{2} \rightarrow \infty} \frac{1}{L_{1} L_{2}} \operatorname{card}\left\{\left(p^{(n)}, \tau^{(n)}\right):\left(p^{(n)}, \tau^{(n)}\right) \in\left(0, L_{1}\right) \times\left(0, L_{2}\right)\right\}=1 .
$$

Similarly, $\left\{U_{n}\right\},\left\{\left(p^{(n)}, \tau^{(n)}\right\}\right.$ satisfy the assumptions of Model B, if $U_{n} \equiv \bar{U}\left(U_{n}\right.$ are constant) and $\left\{\left(p^{(n)}, \tau^{(n)}\right)\right\}$ is a Poisson point process with unit intensity, thus satisfying (B.1).

Before stating and proving the principal results, we recall some useful elementary properties of random fields formed by superposition of "blobs," or elementary wavelets.

Lemma B.1. Let $G(y, s) \in L^{2}\left(\mathbf{R}^{2}\right) \cap L^{1}\left(\mathbf{R}^{2}\right)$ with $\int_{-\infty}^{+\infty} \int_{-\infty}^{+\infty} G(y, s) d y d s=0$. Then, if $\left\{\left(p^{(n)}, \tau^{(n)}\right)\right\},\left\{U_{n}\right\}$, satisfy the assumptions of Models $A$ or $B$, the random variable

$$
Y=\sum_{n} U_{n} G\left(p^{(n)}, \tau^{(n)}\right)
$$

is uniquely defined as an element of $L^{2}(\langle\cdot\rangle)$ and satisfies

$$
\langle Y\rangle=0,\left\langle Y^{2}\right\rangle=\bar{U}^{2} \int_{-\infty}^{+\infty} \int_{-\infty}^{+\infty}(G(y, s))^{2} d y d s
$$

Similarly, the expression

$$
Y(t)=\sum_{n} U_{n} G\left(p^{(n)}+t, \tau^{(n)}+t\right)
$$

defines uniquely a stationary, square-integrable stationary stochastic process for $-\infty<t<\infty$ satisfying $\langle Y(t)\rangle=0$, and

$$
\begin{aligned}
\left\langle Y(t) Y\left(t^{\prime}\right)\right\rangle & =\bar{U}^{2} \iint G(y+t, s+t) G\left(y+t^{\prime}, s+t^{\prime}\right) d y d s \\
& =\bar{U}^{2}(G * G)\left(t-t^{\prime}, t-t^{\prime}\right) .
\end{aligned}
$$

(Here, the symbol $*$ denotes convolution).

This lemma provides a precise definition to the superposition of blobs defined in (B.2) and (B.4) for the case of functions $G(y, s)$ having unbounded support. This 
generality is necessary in order to study random fields with long-range space/time correlations.

Proof of Lemma B.1. We shall give only the proof of the first statement concerning $Y$, since the second one is proved in the same way. Let $\left\{G_{k}(y, s)\right\}$ be a sequence of functions with compact support satisfying

$$
\int_{\mathbf{R}^{2}} \int G_{k}(y, s) d y d s=0
$$

and

$$
\lim _{k \rightarrow \infty} \iint\left(G_{k}(y, s)-G(y, s)\right)^{2} d y d s=0 .
$$

Then $\left\{G_{k}(y, s)\right\}$ is a Cauchy sequence in $L^{2}\left(\mathbf{R}^{2}\right)$, i.e., for all $v>0$ there exists an integer $k(v)$ such that

$$
\left\|G_{k}-G_{k^{\prime}}\right\|_{L^{2}\left(\mathbf{R}^{2}\right)}<v, \text { for } k, k^{\prime}>v
$$

This implies that, for the sequence of random variables

$$
\left.Y_{l} \equiv \sum_{n} U_{n} G_{k}\left(p^{(n)}, \tau^{(n)}\right)\right)
$$

which are well-defined, since $G_{k}$ has compact support [11], satisfies

$$
\begin{aligned}
& \left\langle\left|Y_{k}-Y_{k}^{\prime}\right|^{2}\right\rangle \\
& \quad=\left\langle\sum _ { n } \sum _ { n ^ { \prime } } U _ { n } U _ { n ^ { \prime } } \left( G_{k}\left(p^{(n)}, \tau^{(n)}-G_{k^{\prime}}\left(p^{(n)}, \tau^{(n)}\right) \times\left(G_{k}\left(p^{\left(n^{\prime}\right)}, \tau^{\left(n^{\prime}\right)}\right)-G_{k^{\prime}}\left(p^{\left(n^{\prime}\right)}, \tau^{\left(n^{\prime}\right)}\right)\right)\right\rangle\right.\right. \\
& \quad=\bar{U}^{2}\left\|G_{k}-G_{k^{\prime}}\right\|^{2} \\
& \quad\left\langle\bar{U}^{2} v^{2},\right.
\end{aligned}
$$

for $k, k^{\prime}>k(v)$. Hence, $\left\{Y_{k}\right\}$ is a Cauchy sequence in $L^{2}(\langle\cdot\rangle)$ (the space of squareintegrable random variables, which are measurable with respect to the $\sigma$-algebra generated by $\left.\left\{U_{n}\right\},\left(p^{(n)}, \tau^{(n)}\right), n \geqq 1\right)$. Therefore, $\left\{Y_{k}\right\}$ converges in $L^{2}(\langle\cdot\rangle)$ as $k \rightarrow 0$ to a random variable $Y$ satisfying (B.3). Notice also, that Eq. (B.10) holds for Model A because the $U_{n}$ 's are independent with mean zero, and for Model B because $\left(p^{(n)}, \tau^{(n)}\right)$ is a Poisson process with intensity 1 . This concludes the proof of Lemma B.1.

The following proposition is used to obtain asymptotic expressions for the renormalized particle displacements in regions III, IV, V and III ${ }^{\bar{w}}$.

Proposition B.2. Assume that $\left\{U_{n}\right\},\left\{\left(p^{(n)}, \tau^{(n)}\right)\right\}$ satisfy the assumptions of Model $A$ and $B$, and let $\left\{G_{\delta}(y, s)\right\}_{\delta>0}$ be a sequence of functions in $L^{2}\left(\mathbf{R}^{2}\right) \cap L^{1}\left(\mathbf{R}^{2}\right)$ such that $\iint\left(G_{\delta}(y, s)\right) d y d s=0$ for each $\delta>0$, and such that, for some $G_{0}(y, s) \in L^{2}\left(\mathbf{R}^{2}\right)$, we have

$$
\lim _{\delta \downarrow 0} \int_{-\infty}^{+\infty} \int_{-\infty}^{+\infty}\left(G_{\delta}(y, s)-G_{0}(y, s)\right)^{2} d y d s=0 .
$$

Then, for all $z>0$ the random variables

$$
Y(\delta)=\delta^{1+z / 2} \sum_{n} U_{n} G_{\delta}\left(\delta p^{(n)}, \delta^{z} \tau^{(n)}\right)
$$

converge in distribution to a centered Gaussian random variable with mean zero and 
variance

$$
\sigma^{2}=\bar{U}^{2} \iint\left(G_{0}(y, s)\right)^{2} d y d s .
$$

Proof. Assume first that $G_{\delta}(y, s)=G_{0}(y, s)$ for all $\delta>0$ and that $G_{0} \in C_{0}^{\infty}\left(\mathbf{R}^{2}\right)$. This assumption is removed subsequently by an approximation argument.

We consider first Model A. For a fixed realization of the point process $\left(p^{(n)}, \tau^{(n)}\right)$, the conditional variance of $Y(\delta), \sigma_{\delta}^{2}$, is given by

$$
\sigma_{\delta}^{2} \equiv E\left\{(Y(\delta))^{2} \mid\left(p^{(n)}, t^{(n)}\right)\right\}=\delta^{1+z} \sum_{n} \bar{U}^{2}\left[G_{0}\left(\delta p^{(n)}, \delta^{z} \tau^{(n)}\right)\right]
$$

where the sum extends over all integers $n$ such that $\left(\delta p^{(n)}, \delta^{z} \tau^{(n)}\right)$ lies in the support of the function $G_{0},(y, s)$. From the ergodic theorem, and (B.1), we have

$$
\lim _{\delta \downarrow 0} \delta^{1+z} \sum_{n} \bar{U}^{2}\left(G_{0}\left(\delta p^{(n)}, \delta^{z} \tau^{(n)}\right)\right)^{2}=\bar{U}^{2} \iint\left(G_{0}(y, s)\right)^{2} d y d s,
$$

almost surely. Observe that, given a single realization of $\left(p^{(n)}, \tau^{(n)}\right)_{n}$, the random variables $X_{n}^{(\delta)}=U_{n} G_{0}\left(\delta p^{(n)}, \delta^{2} \tau^{(n)}\right)$ are independent. Moreover, since $G_{0}$ is bounded, and thus $X_{n}^{(\delta)} \approx 0\left(U_{n}\right)$, the sequences $\left\{X_{n}(\delta)\right\}$ satisfy the classical Lindeberg-Levy condition [6], ensuring the validity of the central limit theorem for the sums (B.12). Hence

$$
\lim _{\delta \downarrow 0} E\left\{e^{i \lambda Y(\delta)} \mid\left(p^{(n)}, \tau^{(n)}\right), n \geqq 1\right\}=e^{-\lambda^{2} \sigma^{2} / 2}, \quad \text { almost surely, }
$$

where $\sigma^{2}$ is given by (B.13), for $\lambda$ in a countable dense set of real numbers. Therefore, for all $\lambda$ in such a set, we have

$$
\begin{aligned}
\lim _{\delta \downarrow 0} E\left\{e^{i \lambda Y(\delta)}\right\} & =\lim _{\delta \downarrow 0} E\left\{E\left\{e^{i \lambda Y(\delta)} \mid\left(p^{(n)}, \tau^{(n)}\right\}\right\}\right. \\
& =e^{-\lambda^{2} \sigma^{2} / 2}
\end{aligned}
$$

This implies that $E\left\{e^{i \lambda \bar{y}(\delta)}\right\}$ converges for all $\lambda$ and hence that $Y(\delta)$ converges to a centered Gaussian with variance $\sigma^{2}=\bar{U}^{2} \iint\left(G_{0}(y, s)\right)^{2} d y d s$.

We consider next Model B, i.e. we assume that $U_{n}=\bar{U}$, and that $\left\{\left(p^{(n)}, \tau^{(n)}\right)\right\}$ is a Poisson process with unit intensity. The characteristic function of $Y(\delta)$ can then be computed explicitly. In fact, we have

$$
\begin{aligned}
E\left\{e^{i \lambda y /(\delta)}\right\} & =\exp \left[\delta^{-(1+z)} \iint\left[e^{i \lambda \bar{U} \delta^{(1+z) / 2} G_{0}(y, s)}-1\right]\right] \\
& =\exp \left[\delta^{-(1+z)} \iint\left[e^{i \lambda \bar{U} \delta^{(1+z) / 2} G_{0}(y, s)}-1\right]\right] d y d s .
\end{aligned}
$$

Using the fact that

$$
\begin{aligned}
& \exp \left[i \lambda \bar{U} \delta^{(1+z) / 2} G_{0}(y, s)\right]-1-i \lambda \bar{U} \delta^{(1+z) / 2} G_{0}(y, s)=-\frac{1}{2} \lambda^{2} \bar{U}^{2} \delta^{1+z}\left(G_{0}(y, s)\right)^{2} \\
& \cdot(1+o(1))
\end{aligned}
$$

and that $G_{0}(y, s)$ has mean zero and compact support, we obtain, substituting (B.19) in the preceding Eq. (B.18) and passing to the limit,

$$
\lim _{\delta \downarrow 0} E\left\{e^{i \lambda Y(\delta)}\right\}=\exp \left[\frac{-\lambda^{2} \bar{U}^{2}}{2} \iint\left(G_{0}(y, s)\right)^{2} d y d s\right] .
$$


This concludes the proof of Proposition B. 2 in the case of identical, smooth blobs with compact support.

In the more general case, in which the functions $G_{\delta}(y, s)$ converge strongly to $G_{0}(y, s)$ in $L^{2}\left(\mathbf{R}^{2}\right)$, we argue as follows: first, note that $\left\{G_{\delta}\right\}$ is a Cauchy sequence. Therefore, for all $v=0$ there exists $\delta_{1}>0$ such that for all $\delta>\delta_{1}$ we have

$$
\left\|G_{\delta}-G_{\delta_{1}}\right\|_{L^{2}\left(\mathbf{R}^{2}\right)}<v
$$

and hence

$$
E\left\{\left[Y(\delta)-\delta^{(1+z) / 2} \sum_{n} U_{n} G_{\delta_{1}}\left(\delta p^{(n)}, \delta^{z} \tau^{(n)}\right)\right]^{2}\right\} \leqq \bar{U}^{2} v^{2}
$$

This shows that it is sufficient to establish the proposition for $G_{\delta}(y, s) \equiv G(y, s)$, where $G(y, s) \in L^{2}\left(\mathbf{R}^{2}\right) \cap L^{1}\left(\mathbf{R}^{2}\right)$ and $\int_{-\infty}^{\infty} G(y, s) d y d s=0$. We can further reduce the problem by using the density of $C_{0}^{\infty}\left(\mathbf{R}^{2}\right)$ in the subspace of $L^{2}\left(\mathbf{R}^{2}\right) \cap L^{1}\left(\mathbf{R}^{2}\right)$ of functions with integral equal to zero. Accordingly, if $G^{(v)}(y, s) \in C_{0}^{\infty}\left(\mathbf{R}^{2}\right)$ satisfies $\left\|G-G^{(v)}\right\|_{L^{2}\left(\mathbf{R}^{2}\right)}<v$, then we have

$$
E\left\{\left[Y(\delta)-\delta^{(1+Z) / 2} \sum_{n} U_{n} G^{(v)}\left(\delta p^{(n)}, \delta^{z} \tau^{(n)}\right)\right]^{2}\right\} \leqq \bar{U}^{2} v^{2},
$$

uniformly in the parameters. Hence, it is sufficient to show that $Y(\delta)$ is asymptotically Gaussian when $G_{\delta}(y, s)=G_{0}(y, s)$ is an element of $C_{0}^{\infty}\left(\mathbf{R}^{2}\right)$. Since this was already established, this concludes the proof of Proposition B.2.

Remark. Using the method of proof developed here, one can show that the conclusion of Proposition B.2 is valid if the assumption (B.11) is replaced by

$$
\left|G_{\delta}(y, s)\right| \leqq C \quad \text { for all } \delta>0,
$$

where $C$ is a fixed constant, and

$$
\lim _{\delta \downarrow 0} \int_{-\infty}^{+\infty} \int_{-\infty}^{+\infty}\left(G_{\delta}(y, s)\right)^{2} d y d s=\int_{-\infty}^{+\infty} \int_{-\infty}^{+\infty}\left(G_{0}(y, s)\right)^{2} d y d s .
$$

The following proposition is a central limit theorem which is used to characterize the long-time behavior in regions II and II $^{\bar{w}}$.

Proposition B.3. Assume $\varepsilon>0, z>0$ and $\varepsilon+z>0$. Let $G_{\delta}(y, s)$ be given by

$$
G_{\delta}(y, s)=\frac{1}{2 \pi} \iint \hat{G}_{\delta}(k, \omega) e^{i k y+i \omega s} d k d \omega,
$$

with

$$
\begin{aligned}
\hat{G}_{\delta}(k, \omega) & =\frac{\psi_{0}^{1 / 2}(k) \psi_{\infty}^{1 / 2}(\delta k)|k|^{(1-\varepsilon-z) / 2}}{a^{1 / 2} \pi^{1 / 2}\left(1+\frac{\omega^{2}}{a^{2}|k|^{2 z}}\right)^{1 / 2}} \\
& =\frac{a^{1 / 2} \psi_{0}^{1 / 2}(k) \psi_{\infty}^{1 / 2}(\delta k)|k|^{(1-\varepsilon+z) / 2}}{\pi^{1 / 2}\left(a^{2}|k|^{2 z}+\omega^{2}\right)^{1 / 2}}
\end{aligned}
$$


and define the stationary random process

$$
V_{\delta}(s)=\delta^{(1+z) / 2} \sum_{n} U_{n} G_{\delta}\left(\delta p^{(n)}, \delta^{z} \tau^{(n)}+s\right),
$$

where $\left\{U_{n}\right\}$ and $\left\{\left(p^{(n)}, \tau^{(n)}\right\}\right.$ verify the assumptions of Model A or Model B. Then, for all positive functions $\theta(\delta)$, such that $\lim _{\delta \downarrow 0} \theta(\delta)=+\infty$, the random variables

$$
Y(\delta) \equiv \frac{1}{\sqrt{\theta(\delta)}} \int_{0}^{\theta(\delta)} V_{\delta}(s) d s
$$

converge in distribution to a centered Gaussian with variance

$$
\sigma^{2}=\frac{2 \bar{U}^{2}}{a} \int \psi_{0}(k)|k|^{1-\varepsilon-z} d k .
$$

Proof. The proof proceeds in two steps. First, it is shown that for each positive number $v>0$, there exists a smooth function $\widetilde{G}(y, s)$, with compact support, such that

$$
\int_{-\infty}^{+\infty} \int_{-\infty}^{+} \tilde{G}(y, s) d y d s=0
$$

with the property that, if we define the process

$$
\tilde{V}_{\delta}(s)=\delta^{(1+z) / z} \sum_{n} U_{n} \tilde{G}\left(\delta p^{(n)}, \delta^{z} \tau^{(n)}+s\right)
$$

and set

$$
\tilde{Y}(\delta)=\frac{1}{\sqrt{\theta(\delta)}} \int_{0}^{\theta(\delta)} \tilde{V}_{\delta}(s) d s,
$$

then the asymptotic variance of $Y(\delta)-\tilde{Y}(\delta)$ is bounded by $v$, i.e.,

$$
\varlimsup_{\delta \downarrow 0}\left\langle|Y(\delta)-\tilde{Y}(\delta)|^{2}\right\rangle \leqq v .
$$

The second step consists in showing that $\tilde{Y}(\delta)$ is equal to a normalized sum of "2-dependent" random variables satisfying the classical Lyapunov $4^{\text {th }}$ moment condition for asymptotic normality $[6,11]$. This implies that $\widetilde{Y}(\delta)$ is asymptotically normal, and, since the asymptotic variance of $Y(\delta)-\tilde{Y}(\delta)$ can be made arbitrarily small, the conclusion follows.

Step 1. Let us fix $\delta_{0}>0$. For each $\delta>0$, set

$$
V_{\delta}^{(0)}=\delta^{(1+z) / 2} \sum_{n} U_{n} G_{\delta_{0}}\left(\delta p^{(n)}, \delta^{z} \tau^{(n)}+s\right)
$$

and

$$
Y^{(0)}(\delta)=\frac{1}{\sqrt{\theta}(\delta)} \int_{0}^{\theta(\delta)} V_{\delta}^{(0)}(s) d s
$$


Notice that $\left\langle Y^{(0)}(\delta)\right\rangle=\langle Y(\delta)\rangle=0$, for all $\delta>0$. An explicit calculation shows that

$$
\begin{aligned}
\lim _{\delta \downarrow 0}\left\langle\left|Y(\delta)-Y^{0}(\delta)\right|^{2}\right\rangle= & \lim _{\delta \downarrow 0} 2 \bar{U}^{2} \int \psi_{0}(k)\left[\psi_{\infty}(\delta k)^{1 / 2}-\psi_{\infty}\left(\delta_{0} k\right)^{1 / 2}\right]|k|^{1-\varepsilon} \\
& \cdot\left(\frac{1}{\theta(\delta)} \int_{0}^{\theta(\delta)} d s \int_{0}^{s} e^{-a|k| s^{\prime}} d s^{\prime}\right) d k . \\
& +\frac{2 \bar{U}^{2}}{a} \int_{-\infty}^{+\infty} \psi_{0}(k)\left[1-\psi_{\infty}^{1 / 2}\left(\delta_{0} k\right)\right]|k|^{1-\varepsilon-z} d k .
\end{aligned}
$$

Hence, the asymptotic variance of $\left.Y(\delta)-Y^{(0)}(\delta)\right)$ can be made arbitrarily small by choosing $\delta_{0}$ small enough. Consequently, if $Y^{(0)}(\delta)$ is asymptotically normal for all $\delta_{0}$, as $\delta \rightarrow 0$, then the same is true for $Y(\delta)$. This reduces the problem to the study of the single blob $G_{\delta_{0}}(y, s)$, which has the property that its Fourier transform, $\hat{G}_{\delta_{0}}(k, \omega)$ is smooth and vanishes for $|k|<k_{0}$ or $|k|>k_{1}$, where $k_{0}, k_{1}$ are positive constants. For this reason, the partial derivatives

$$
\left(\frac{\partial}{\partial k}\right)^{p}\left(\frac{\partial}{\partial \omega}\right)^{q} \hat{G}_{\delta_{0}}(k, \omega)=\left(\frac{\partial}{\partial k}\right)^{p}\left(\frac{\partial}{\partial \omega}\right)^{q} \frac{a^{1 / 2} \psi_{0}(k)^{1 / 2} \psi_{\infty}\left(\delta_{0} k\right)^{1 / 2}|k|^{(1-\varepsilon+z) / 2}}{\pi^{1 / 2}\left(a^{2}|k|^{2 z}+\omega^{2}\right)^{1 / 2}}
$$

are square-integrable for all $p>0, q>0$ and hence $|y|^{p}|s|^{q}\left|G_{\delta_{0}}(y, s)\right|$ is squareintegrable for all positive integers $p, q$. From this, we conclude that for all $v>0$, there exists $\widetilde{G}(y, s)$ smooth, with compact support, such that (B.29) holds and

$$
\int_{-\infty}^{+\infty} d s\left(\int_{-\infty}^{+\infty}\left|G_{\delta_{0}}(y, s)-\tilde{G}(y, s)\right|^{2} d y\right)^{1 / 2}<\frac{v^{1 / 2}}{\bar{U} \sqrt{2}} .
$$

The asymptotic variance of $\left(Y^{(0)}(\delta)-Y(\delta)\right)$ is given by

$$
\begin{aligned}
\lim _{\delta \downarrow 0}\left\langle\left|Y^{(0)}(\delta)-\tilde{Y}(\delta)\right|^{2}\right\rangle & =\lim _{\delta \downarrow} \frac{2}{\theta(\delta)} \int_{0}^{\theta(\delta)} d s \int_{0}^{s}\left\langle\left(V_{\delta}^{(0)}(s)-\tilde{V}_{\delta}(s)\right)\left(V_{\delta}^{(0)}\left(s^{\prime}\right)-\tilde{V}_{\delta}\left(s^{\prime}\right)\right)\right\rangle d s^{\prime} \\
& =\lim _{\delta \downarrow 0} \frac{2 \bar{U}^{2}}{\theta(\delta)} \int_{0}^{\theta(\delta)} d s \int_{0}^{s}\left(G_{\delta_{0}}-\tilde{G}\right)^{*}\left(G_{\delta_{0}}-\tilde{G}\right)\left(0, s^{\prime}\right) d s^{\prime} \\
& =2 \bar{U}^{2} \int_{-\infty}^{+\infty}\left[\int_{-\infty}^{+\infty}\left(G_{\delta_{0}}(y, s)-\tilde{G}(y, s)\right) d s\right]^{2} d y \\
& =2 \bar{U}^{2}\left(\int_{-\infty}^{+\infty}\left(\int_{-\infty}^{+\infty}\left|G_{\delta_{0}}(y, s)-\tilde{G}(y, s)\right|^{2} d y\right)^{1 / 2} d s\right)^{2} \\
& <v .
\end{aligned}
$$

Since $v$ can be arbitrarily small, this calculation shows that it is sufficient to establish the asymptotic normality of $\widetilde{Y}(\delta)$ as $\delta \rightarrow 0$, for arbitrary smooth functions $\widetilde{G}(y, s)$ with compact support and mean zero. This completes the proof of Step 1.

Finally, we remark that, without loss of generality, the random variables $\left\{U_{n}\right\}$ can be assumed to be uniformly bounded. In fact, let $U_{n}^{*}=\max \left(-M,\left(\min \left(U_{n}, M\right)\right)-C\right.$, where $M$ is a large positive constant and $C$ is chosen so that $\left\langle U_{n}^{*}\right\rangle=0$. The reader will readily verify that asymptotic variance of the difference between $(\theta(\delta))^{-1 / 2} \int_{0}^{\theta(\delta)} V_{\delta}(s) d s$ 
and $\theta(\delta)^{-1 / 2} \int_{0}^{\theta(\delta)} V_{\delta}^{*}(s) d s$, where $V_{\delta}^{*}(s)=\delta^{(1+z) / 2} \sum_{n} U_{n}^{*} G_{\delta}\left(\delta p^{(n)}, \delta^{z} \tau^{(n)}+s\right)$, can be made arbitrarily small by choosing $M$ large enough.

Step 2. We consider first Model $A\left(U_{n}\right.$ independent, identically distributed, $\left(p^{(n)}, \tau^{(n)}\right)$ ergodic). Suppose that $\tilde{G}(y, s)$ vanishes for $|s|>\frac{L}{2}$, where $L$ is a given, positive number. Let us fix a realization $\left(p^{(n)}, \tau^{(n)}\right)$ of the ergodic point process. Then, for all $s, s^{\prime}$ such that $\left|s-s^{\prime}\right|>L$, the random variables $V_{\delta}(s)$ and $V_{\delta}\left(s^{\prime}\right)$ are independent. This a consequence of the fact that the variables $\left\{U_{n}\right\}$ are independent, and that if, for some $n \geqq 1$, the variables $U_{n}$ gives a non-zero contribution to $V_{\delta}(s)$, then $U_{n}$ cannot contribute to $V_{\delta}\left(s^{\prime}\right)$, since $\left|\left(\delta^{z} \tau^{(n)}+s\right)-\left(\delta^{z} \tau^{(n)}+s^{\prime}\right)\right|=\left|s-s^{\prime}\right|>L$ and thus either $\widetilde{G}\left(\delta p^{(n)}, \delta^{z} \tau^{(n)}+s\right)$ or $\widetilde{G}\left(\delta p^{(n)}, \delta^{z} \tau^{(n)}+s^{\prime}\right)$ must vanish. Defining the triangular array of random variables

$$
\begin{aligned}
X_{m}(\delta) & =\frac{1}{\sqrt{L}} \int_{0}^{L} \tilde{V}_{\delta}(s) d s \\
& \equiv \delta^{(1+z) / 2} \sum_{n} U_{n} \frac{1}{\sqrt{L}} \int_{0}^{L} \tilde{G}\left(\delta p^{(n)}+m L, \delta^{z} \tau^{(n)}+m L+s\right) d s \\
& =\delta^{(1+z) / 2} \sum_{n} U_{n} \tilde{H}\left(\delta p^{(n)}+m L, \delta^{z} \tau^{(n)}+m L\right),
\end{aligned}
$$

where

$$
\tilde{H}(y, s)=\frac{1}{\sqrt{L}} \int_{0}^{L} \tilde{G}(y, s+\sigma) d \sigma
$$

we see that $X_{m}(\delta), X_{m^{\prime}}(\delta)$ are independent (given $\left\{\left(p^{(u)}, \tau^{(n)}\right)\right\}$ ) for $\left|m-m^{\prime}\right| \geqq 2$. Notice that for all $N>0$, we have

$$
\frac{1}{\sqrt{N}} \sum_{m=0}^{N-1} X_{m}(\delta)=\frac{1}{\sqrt{N} L} \int_{0}^{N L} V_{\delta}(s) d s
$$

We set $N=N(\delta)=[\theta(\delta) / L]$. Also, the conditional variance, $\sigma^{2}(\delta)$, of the normalized sum (B.42) given $\left\{\left(p^{(n)}, \tau^{(n)}\right)\right\}$ satisfies

$$
\begin{aligned}
\lim _{\delta \downarrow 0} \sigma^{2}(\delta) & =\lim _{\delta \downarrow 0} \frac{2 \bar{U}^{2}}{N(\delta)} \int_{0}^{N(\delta)} d s \int_{0}^{s}\left(\delta^{1+z} \sum_{n} \tilde{G}\left(\delta p^{(n)}, \delta^{z} \tau^{(n)}+s^{\prime}\right) \tilde{G}\left(\delta p^{(n)}, \delta^{(z)} \tau^{(n)}\right)\right) d s^{\prime} \\
& =\lim _{\delta \downarrow 0} \frac{2 \bar{U}^{2}}{N(\delta)} \int_{0}^{N(\delta)} d s \int_{0}^{L}\left(\delta^{1+z} \sum_{n} \tilde{G}\left(\delta p^{(n)}, \delta^{z} \tau^{(n)}+s^{\prime}\right) \tilde{G}\left(\delta p^{(n)}, \delta^{z} \tau(u)\right)\right) \\
& =\lim _{\delta \downarrow 0} 2 \bar{U}^{2} \delta^{1+z} \sum_{n} \int_{0}^{L} \tilde{G}\left(\delta p^{(n)}, \delta^{z} \tau^{(n)}+s^{\prime}\right) \tilde{G}\left(\delta p^{(n)}, \delta^{z} \tau^{(n)}\right) d s^{\prime} \\
& =2 \bar{U}^{2} \int_{0}^{L}(\tilde{G} * \tilde{G})(0, s) d s=2 \bar{U}^{2} \int_{0}^{\infty}(\tilde{G} * \tilde{G})(0, s) d s .
\end{aligned}
$$

Hence, by a well-known central limit theorem for " $m$-dependent" variables [33], the normalized sums (B.42), with $N=N(\delta)$ converge in distribution to a centered 
Gaussian random variable, if the following Lyapunov condition holds:

$$
\lim _{\delta \downarrow 0} \frac{1}{N(\delta)^{2}} \sum_{m=0}^{N(\delta)-1} E\left\{\left(X_{m}(\delta)\right)^{4} \mid\left\{\left(p^{(n)}, \tau^{n}\right)\right\}\right\}=0 .
$$

Here $E\left\{\cdot \mid\left\{\left(p^{(n)}, \tau^{(n)}\right)\right\}\right.$ denotes the conditional expectation, given a realization of the point process, i.e., averaging only over $\left\{u_{n}\right\}$. We show first that (B.44) holds in probability, by establishing that

$$
\left\langle\left|X_{0}(\delta)\right|^{4}\right\rangle \leqq C<\infty,
$$

(where brackets denote averaging over both $\left\{U_{n}\right\}$ and $\left(p^{(n)}, \tau^{(n)}\right)$ ) for some constant independent of $\delta$, and hence that

$$
\lim _{\delta \downarrow 0}\left\langle\frac{1}{N(\delta)^{2}} \sum_{m=0}^{N(\delta)-1} X_{n}(\delta)^{4}\right\rangle=\lim _{\delta \downarrow 0} \frac{1}{N(\delta)}\left\langle\left|X_{0}(\delta)\right|^{4}\right\rangle=0 .
$$

To prove (B.45), notice that the fourth moment of $X_{0}(\delta)$ is given by

where

$$
\left\langle\left|X_{0}(\delta)\right|^{4}\right\rangle=\delta^{2(1+z)}\left\langle U_{1}^{4}\right\rangle\left\langle\sum_{n} H_{n}^{4}\right\rangle+\delta^{2(1+z)}\left\langle U_{1}^{2}\right\rangle^{2}\left\langle\sum_{n \neq n^{\prime}} H_{n}^{2} H_{n^{\prime}}^{2}\right\rangle,
$$

$$
H_{n}=\frac{1}{\sqrt{L}} \int_{0}^{L} G_{0}\left(\delta p^{n}, \delta^{z} \tau^{(n)}+s\right) d s \equiv \tilde{H}\left(\delta p^{(n)}, \delta^{z} \tau^{(n)}\right) .
$$

Therefore,

$$
\begin{aligned}
\left\langle\left|X_{0}(\delta)\right|^{4}\right\rangle= & \delta^{2(z+1)}\left(\left\langle U_{1}^{4}\right\rangle-\left\langle U_{1}^{2}\right\rangle^{2}\right)\left\langle\sum_{n} H_{n}^{4}\right\rangle+\left\langle U_{1}^{2}\right\rangle^{2}\left\langle\left|\delta^{z+1} \sum_{n} H_{n}^{2}\right|^{2}\right\rangle \\
\leqq & \delta^{z+1}\left(\left\langle U_{1}^{4}\right\rangle-\left\langle U_{1}^{2}\right\rangle^{2}\right)\left(\int_{-\infty}^{+\infty} \int_{-\infty}^{+\infty} \tilde{H}^{4}(y, s) d y d s\right) \\
& +\left\langle U_{1}^{2}\right\rangle^{2}\left(\int_{-\infty}^{+\infty}(\tilde{H}(y, s))^{2} d y d s\right)^{2} .
\end{aligned}
$$

We conclude that $\left\langle\left|X_{0}(\delta)\right|^{4}\right\rangle$ is uniformly bounded and hence that (B.45) holds. In particular, the sum of the conditional fourth moments,

$$
F_{\delta}\left\{\left(p^{n}, \tau^{n}\right)\right\}=\frac{1}{N(\delta)^{2}} \sum_{m=0}^{N(\delta)-1} E\left\{X_{m}(\delta)^{4} \mid\left(p^{(n)}, \tau^{(n)}\right)\right\}
$$

converges to zero in probability. Hence there exists a sequence $\delta_{j} \downarrow 0$ such that $F_{\delta}\left\{\left(p^{(n)}, \tau^{(n)}\right\}\right.$ converges to zero in a set of full measure in the space of configurations of the point process $\left(p^{(n)}, \tau^{(n)}\right)$. We conclude that the normalized $Y\left(\delta_{j}\right)$, with $N\left(\delta_{j}\right)=\left[\theta\left(\delta_{j}\right) / L\right]$, satisfy

$$
\lim _{\delta_{j} \rightarrow 0} E\left\{e^{i \lambda Y\left(\delta_{j}\right)} \mid\left(p^{(n)}, \tau^{n}\right)\right\}=\bar{e}^{\left(\lambda^{2} \sigma^{2}\right) / 2}
$$

almost surely, for $\lambda$ in a countable, dense set of $\mathbf{R}$, with

$$
\sigma^{2}=2 \bar{U}^{2} \int_{0}^{\infty}(\tilde{G} * \tilde{G})(0, s) d s
$$


Integrating (B.51) with respect to $\left(p^{(n)}, \tau^{(n)}\right)$ statistics, and using the Dominated Convergence Theorem, we have

$$
\lim _{\delta_{J} \downarrow 0}\left\langle e^{i \lambda Y\left(\delta_{j}\right)}\right\rangle=\bar{e}^{-\left(\lambda^{2} \sigma^{2}\right) / 2},
$$

for a countable dense set of $\lambda$ 's, and hence for all $\lambda \in \mathbf{R}$. Since the family of random variables is tight (because $\left\langle Y(\delta)^{2}\right\rangle$ is uniformly bounded), every sequence $\left\{Y\left(\delta_{j}\right)\right\}$ has a subsequence $\left\{Y\left(\delta_{j_{n}}\right)\right\}$ converging to a centered Gaussian with variance $\sigma^{2}$. We conclude from this that the entire family $\{Y(\delta)\}$ converges to this distribution. This concludes the proof of the proposition for Model A.

The corresponding result for Model B is proved in a similar fashion, observing that for $\left|s-s^{\prime}\right|>L$ the functions $G(\cdot, \cdot+s)$ and $G\left(\cdot, \cdot+s^{\prime}\right)$ have disjoint supports and hence, since $\left(\delta p^{(n)}, \delta^{z} \tau^{(n)}\right)$ is a Poisson point process, the corresponding random variables $V_{\delta}(s)$ and $V_{\delta}\left(s^{\prime}\right)$ are independent.

The next proposition is used to characterize the long-time behavior corresponding region $\mathrm{IV}^{\bar{w}}$.

Proposition B.4. Suppose that $\bar{w} \neq 0$, and that $\varepsilon$, zatisfy the inequalities $1<z<\varepsilon<2$. Define $G_{\delta}(y, s)=(2 \pi)^{-1} \iint \hat{G}_{\delta}(k, \omega) e^{i k y+i \omega s} d k d \omega$ by its Fourier transform

$$
\widehat{G}_{\delta}(k, \omega)=\frac{a^{1 / 2} \psi_{0}^{1 / 2}(k) \psi_{\infty}^{1 / 2}(\delta k)|k|^{(1-\varepsilon+z) / 2}}{\pi^{1 / 2}\left[a^{2} \delta^{2(z-1)}|k|^{2 z}+\omega^{2}\right]^{1 / 2}} .
$$

Let $V_{\delta}(s)$ be the random process defined by

$$
V_{\delta}(s)=\delta \sum_{n} G_{\delta}\left(\delta p^{(n)}+\bar{w} s, \delta \tau^{(n)}+s\right)
$$

where $\left\{U_{n}\right\},\left\{\left(p^{(n)}, \tau^{(n)}\right)\right\}$ satisfy the assumptions of Models A or B. Then, if $\theta(\delta) \rightarrow \infty$ as $\delta \rightarrow 0$, the random variables

$$
Y(\delta) \equiv \frac{1}{\sqrt{\theta}(\delta)} \int_{0}^{\theta(\delta)} V_{\delta}(s) d s
$$

converge in distribution as $\delta \rightarrow 0$ to a centered Gaussian with variance

$$
\sigma^{2}=2 \frac{\bar{U}^{2} a}{\bar{w}^{2}} \int_{-\infty}^{+\infty} \psi_{0}(k)|k|^{-1+\varepsilon-z} d k .
$$

Proof. The proof consists in reducing the problem to the study of a single "blob," independent of $\delta$, having compact support. This enables us to invoke, as in Step 2 of the proof of Proposition B.3, a Central Limit Theorem for sums of 2-dependent random variables.

In a first of a series of reductions, we observe that it is sufficient to the asymptotic normality of modified sequence of random variables, $Y^{(1)}(\delta)$, obtained by considering, instead of $G_{\delta}(y, s)$, the blobs

with

$$
G_{\delta}^{(1)}(y, s)=\frac{1}{2 \pi} \iint \hat{G}_{\delta}^{(1)}(k, \omega) e^{i k y+i \omega s} d k d \omega
$$

$$
\hat{G}_{\delta}^{(1)}(k, \omega)=\frac{a^{1 / 2} \psi_{0}^{1 / 2}(k) \psi_{\infty}^{1 / 2}\left(\delta_{1} k\right)|k|^{(1-\varepsilon+z) / 2}}{\pi^{1 / 2}\left[a^{2} \delta^{2(z-1)}|k|^{2 z}+\omega^{2}\right]^{1 / 2}}
$$


In this last equation, notice that $\hat{G}_{\delta}^{(1)}(k, \omega)$ vanishes for $|k|<k_{0}$ and $|k|>k_{1}$, where $k_{0}, k_{1}$ are positive constants which are independent of $\delta$. The justification of this reduction is done by explicitly computing the asymptotic variance of $Y(\delta)-Y^{(1)}(\delta)$ and showing that it can be made arbitrarily small by an appropriate choice of the parameter $\delta_{1}$ in (B.59). This explicit calculation is possible because (B.54) and (B.59) have the same form, as functions of $\omega$.

Due to the presence of the small parameter $\delta^{2(z-1)}$ in the denominator of $\hat{G}_{\delta}^{(1)}(k, \omega)$, this function becomes singular as $\delta \rightarrow 0$. For this reason, we make a second approximation, introducing the function $G_{\delta}^{(2)}(y, s)$, with Fourier transform

$$
\begin{aligned}
\hat{G}_{\delta}^{(2)}(k, \omega) & =\frac{a^{1 / 2} \psi_{0}^{1 / 2}(k) \psi_{\infty}^{1 / 2}\left(\delta_{1} k\right) \psi_{0}^{1 / 2}\left(\omega / \delta_{2}\right) k^{(1-\varepsilon+z) / 2}}{\pi^{1 / 2}\left[a^{2} \delta^{2(z-1)}|k|^{2 z}+\omega^{2}\right]^{1 / 2}}, \\
& =\psi_{0}^{1 / 2}\left(\frac{\omega}{\delta_{2}}\right) \hat{G}_{\delta}^{(1)}(k, \omega),
\end{aligned}
$$

where $\delta_{2}$ is a fixed constant. This new function vanishes identically for $|\omega| \leqq c \delta_{2}$. This implies, using the fact that the function $\hat{G}_{\delta}^{(2)}(k, \omega)$ is band-limited in $k$, that the derivatives $D_{\omega}^{p} D_{k}^{q} \hat{G}_{\delta}^{(2)}(k, \omega)$ are uniformly bounded in $L^{2}\left(\mathbf{R}^{2}\right)$, for all positive integers $p$ and $q$. Hence, $|s|^{p}|y|^{q} G_{\delta}^{(2)}(y, s) \leqq C_{p q}$ for some constant $C_{p, q}$, uniformly in the small parameter $\delta$. We claim that, for an appropriate choice of the small parameter $\delta_{2}$, the functions $G_{\delta}^{(2)}(y, s)$ give rise to normalized sums $Y^{(2)}(\delta)$ (defined according to (B.55), with $G_{\delta}^{(2)}$ in place of $\left.G_{\delta}\right)$, such that $\left(Y^{(2)}(\delta)-Y^{(1)}(\delta)\right)$ has an arbitrarily small asymptotic variance. To see this, define the function

and observe that

$$
\zeta(\omega)=1-\psi_{0}^{1 / 2}\left(\frac{\omega}{\delta_{2}}\right),
$$

$$
\begin{aligned}
& \left\langle\left[Y^{(2)}(\delta)-Y^{(1)}(\delta)\right]^{2}\right\rangle \\
& =2 \lim _{\delta \downarrow 0} \frac{1}{\theta(\delta)} \int_{0}^{\theta(\delta)} d s \int_{0}^{s} d s^{\prime}\left\langle\left(V_{\delta}^{(2)}\left(s^{\prime}\right)-V_{\delta}^{(1)}\left(s^{\prime}\right)\right)\left(V_{\delta}^{(2)}(0)-V_{\delta}^{(1)}(0)\right\rangle d s^{\prime}\right. \\
& =2 \bar{U}^{2} \lim _{\delta \downarrow} \frac{1}{\theta(\delta)} \int_{0}^{\theta(\delta)} d s \int_{0}^{s} d s^{\prime}\left(G_{\delta}^{(2)}-G_{\delta}^{(1)}\right)^{*}\left(G_{\delta}^{(2)}-G_{\delta}^{(1)}\right)\left(\bar{w} s^{\prime}, s^{\prime}\right) \\
& =2 \bar{U}^{2} \lim _{\delta \downarrow 0} \frac{1}{\theta(\delta)} \int_{0}^{\theta(\delta)} d s \int_{0}^{s} d s^{\prime}\left[\iint\left|\hat{G}_{\delta}^{(2)}-\hat{G}_{\delta}^{(1)}\right|^{2}(k, \omega) e^{i k \bar{w} s^{\prime}+i \omega s^{\prime}} d k d \omega\right] \\
& =2 \bar{U}^{2} \lim _{\delta \downarrow 0} \frac{1}{\theta(\delta)} \int_{0}^{\theta(\delta)} d s\left[\iint\left|\hat{G}_{\delta}^{(2)}(k, \omega)-\hat{G}_{\delta}^{(1)}(k, \omega)\right|^{2} \frac{\sin (k \bar{w}+\omega) s}{(k \bar{w}+\omega)} d k d \omega\right] \\
& =2 \bar{U}^{2} \lim _{\delta \downarrow 0} \frac{1}{\theta(\delta)} \int_{0}^{\theta} F_{\delta}(s) d s,
\end{aligned}
$$

where

$$
\begin{aligned}
F_{\delta}(s) & =\iint\left|\hat{G}_{\delta}^{(2)}(k, \omega-k \bar{w})-\hat{G}_{\delta}^{(1)}(k, \omega-k \bar{w})\right|^{2} \frac{\sin \omega s}{\omega} d k d \omega \\
& =\frac{a}{\pi} \iint \frac{\psi_{0}(k) \psi_{\infty}\left(\delta_{1} k, \zeta^{2}(\omega-k \bar{w})|k|^{1-\varepsilon+z}\right.}{a^{2} \delta^{2(z-1)}|k|^{2 z}+|\omega-k \bar{w}|^{2}} \frac{\sin \omega s}{\omega} d k d \omega .
\end{aligned}
$$


We write the limit in (B.62) as

$$
\lim _{\delta \downarrow 0} \iint \frac{a \psi_{0}(k) \psi_{\infty}\left(\delta_{1} k\right) \zeta^{2}(\omega-k \bar{w})|k|^{1-\varepsilon+z}}{\pi\left(a^{2} \delta^{2(z-1)}|k|^{2 z}+|\omega-k \bar{w}|^{2}\right)}\left[\frac{1-\cos \omega \theta(\delta)}{\theta(\delta) \omega^{2}}\right] d k d \omega .
$$

Since the function $\frac{1-\cos \omega \theta(\delta)}{\theta(\delta) \omega^{2}}$, in non-negative, the latter expression is bounded by

$$
\begin{aligned}
& \lim _{\delta \downarrow 0} \iint \frac{a \psi_{0}(k) \psi_{\infty}\left(\delta_{1} k\right) \zeta^{2}(\omega-k \bar{w})|k|^{1-\varepsilon+z}}{\pi|\omega-k \bar{w}|^{2}}\left(\frac{1-\cos \omega \theta(\delta)}{\omega^{2} \theta(\delta)}\right) d k d \omega \\
& \quad=\lim _{\delta \downarrow 0} \frac{1}{\theta(\delta)} \int_{0}^{\theta(\delta)} F_{0}(s) d s,
\end{aligned}
$$

where the function $F_{0}(s)$ is obtained by setting $\delta=0$ in (B.63), i.e.,

$$
F_{0}(s)=\frac{a}{\pi} \iint \frac{\psi_{0}(k) \psi_{\infty}\left(\delta_{1} k\right) \zeta^{2}(\omega-k \bar{w})|k|^{1-\varepsilon+z}}{|\omega-k \bar{w}|^{2}} \frac{\sin \omega s}{\omega} d k d \omega
$$

We now choose the parameter $\delta_{2}$ in the cutoff function $\zeta(\omega)=1-\psi_{0}^{1 / 2}\left(\frac{\omega}{\delta_{2}}\right)$ so that the supports of $\zeta^{2}(-\bar{w} k)$ and $\psi_{0}(k)$ are disjoint. This is always possible if $\delta_{2}$ is small enough. In the latter case, the following property must hold: there exists a positive number $\omega_{0}$, such that

$$
\left\{\begin{array}{l}
\text { if } \quad \psi_{0}(k)>0 \quad \text { and } \quad \zeta^{2}(\omega-k \bar{w})>0, \\
\text { then }|\omega| \geqq \omega_{0} .
\end{array}\right.
$$

On account of (B.67), the function

$$
\frac{\psi_{0}(k) \psi_{\infty}\left(\delta_{1} k\right) \zeta^{2}(\omega-k \bar{w})|k|^{1-\varepsilon+z}}{|\omega-k \bar{w}|^{2} \omega}
$$

is integrable, so that by the classical Riemann-Lebesgue lemma, we have

$$
\lim _{s \rightarrow \infty} F_{0}(s)=0
$$

and hence

$$
\lim _{\delta \downarrow}\left\langle\left[Y^{(2)}(\delta)-Y^{(1)}(\delta)\right]^{2}\right\rangle=0 .
$$

Finally, we claim that the asymptotic error is negligible if $G_{\delta}^{(2)}(y, s)$ is replaced by the fixed blob $G^{(3)}(y, s)$ with the Fourier transform obtained by setting $\delta=0$ in (B.60), i.e.,

$$
\hat{G}^{(3)}(k, \omega)=\frac{\alpha^{1 / 2} \psi_{0}^{1 / 2}(k) \psi_{\infty}^{1 / 2}\left(\delta_{1} k\right) \psi_{0}^{1 / 2}\left(\frac{\omega}{\delta_{3}}\right)|k|^{(1-\varepsilon-z) / 2}}{\pi^{1 / 2}|\omega|} .
$$

In fact, the asymptotic variance of the difference between the corresponding random 
variables $Y^{(2)}(\delta)$ and $Y^{(3)}(\delta)$ is given by

$$
\begin{aligned}
& \lim _{\delta \downarrow 0}\left\langle\left|Y^{(2)}(\delta)-Y^{(3)}(\delta)\right|^{2}\right\rangle \\
& \quad=2 \bar{U}^{2} \lim _{\delta \downarrow 0} \iint\left|\hat{G}_{\delta}^{(2)}(k, \omega-k \bar{w})-\hat{G}^{(3)}(k, \omega-k \bar{w})\right|^{2}\left(\frac{1-\cos \omega \theta(\delta)}{\omega^{2} \theta(\delta)}\right) d k d \omega .
\end{aligned}
$$

This last integral is dominated by

$$
\begin{aligned}
& \delta^{2(z-1)} \frac{a^{3}}{\pi} \iint \frac{\psi_{0}(k) \psi_{\infty}\left(\delta_{1} k\right) \psi_{0}\left(\frac{w-k \bar{w}}{\delta_{2}}\right)|k|^{1-\varepsilon+3 z}}{|\omega-k \bar{w}|^{2}} \frac{1-\cos \omega \theta(\delta)}{\omega^{2} \theta(\delta)} d k d \omega \\
& \equiv \frac{\delta^{2(z-1)} a^{3}}{\pi} \frac{1}{\theta(\delta)} \int_{0}^{\theta(\delta)} \tilde{F}(s) d s,
\end{aligned}
$$

where the auxiliary function $\tilde{F}(s)$ is defined by

$$
\tilde{F}(s)=\iint\left[\frac{\psi_{0}(k) \psi_{\infty}\left(\delta_{1} k\right) \psi_{0}\left(\frac{\omega-k \bar{w}}{\delta_{2}}\right)|k|^{1-\varepsilon+3 z}}{|\omega-k \bar{w}|^{2}}\right] \frac{\sin \omega t}{\omega} d k d \omega .
$$

The function in brackets in this integral is easily seen to be smooth and integrable. Hence,

$$
\lim _{\delta \downarrow 0} \tilde{F}_{\delta}(s)=\pi \int_{-\infty}^{+\infty} \psi_{0}(k) \psi_{\infty}\left(\delta_{1} k\right) \psi_{0}\left(\frac{-k \bar{w}}{\delta_{2}}\right)|k|^{-1-\varepsilon+3 z} d k
$$

and from (B.74), we conclude that $\left\langle\left[Y^{(3)}(\delta)-Y^{(2)}(\delta)\right]^{2}\right\rangle$ converges to zero as $\delta \rightarrow 0$.

We have reduced the problem to the study of a single blob, $G^{(3)}(y, s)$, independent of $\delta$, which is smooth and decays rapidly at infinity. The asymptotic variance of $Y^{(3)}(\delta)$ is

$$
\lim _{\delta \downarrow 0}\left\langle\left[Y^{(3)}(\delta)\right]^{2}\right\rangle=2 \bar{U}^{2} \int_{0}^{\infty}\left(G^{(3)} * G^{(3)}\right)(\bar{w} s, s) d s,
$$

where $*$ denotes convolution in $y$ and $s$. Given a small positive number $v$, let $\tilde{G}(y, s)$ be a smooth function with compact support satisfying

$$
\int_{0}^{-\infty}\left(G^{(3)}-\tilde{G}\right) *\left(G^{(3)}-\tilde{G}\right)(\bar{w} s, s) d s<\frac{v}{2 \bar{U}^{2}}
$$

and

$$
\iint \tilde{G}(y, s) d y d s=0
$$

It is easy to verify that the asymptotic variance of the corresponding normalized sums satisfies

$$
\lim _{\delta \downarrow 0}\left\langle\left[Y^{(3)}(\delta)-\tilde{Y}(\delta)\right]^{2}\right\rangle=2 \bar{U}^{2} \int_{0}^{\infty}\left(G^{(3)}-\tilde{G}\right) *\left(G^{(3)}-\tilde{G}\right)(\bar{w} s, s) d s<v .
$$

We have therefore shown that the conclusion of Proposition 4 follows if we establish 
the asymptotic normality of normalized integrals (B.56) corresponding to compactly supported blobs with mean zero. The proof of this latter fact is done in the same way as Step 2, Proposition B.3, using the fact that the corresponding random processes $\widetilde{V}_{\delta}(s)$ have finite domain of dependence. This concludes the proof of Proposition B.4.

\section{References}

1. Avellaneda, M., Majda, A. J.: Commun. Math. Phys. 131, 381-429 (1990)

2. Avellaneda, M., Majda, A. J.: Physics of Fluids A 4(1), 41-57 (1992)

3. Avellaneda, M., Majda, A. J.: In: The dynamical systems approach to the theory of turbulence in fluid flows. Foias, C., Sell, G., Temam, R. (eds.). Institute for Mathematics and its Applications. Berlin, Heidelberg, New York: Springer 1991 (in press)

4. Avellaneda, M., Majda, A. J.: Commun. Math. Phys., 138, 339-391 (1991)

5. Avellaneda, M., Majda, A. J.: Superballistic renormalization for turbulent transport. In: preparation

6. Avellaneda, M., Majda, A. J.: Renormalization theory for Eddy diffusivity in turbulent transport. Submitted to Phys. Rev. Lett., 1991

7. Boussoussan, A., Lions, J.-L., Papanicolaou, G. C.: Asymptotic analysis of periodic structures. Amsterdam: North-Holland 1978

8. Billingsley, P.: Convergence of probability measures. New York: John Wiley 1968

9. Borodin, A. N.: Theory Probab. Appl. 22 (3), 482-497 (1978)

10. Bouchaud, J. P., Comptet, A., Georges, A., Le Doussal, P.: J. Phys. (Paris) 48, 1445 (1987)

11. Bouchaud, J. P., Georges, A., Koplik, J., Provata, A., Redner, S.: Phys. Rev. Lett. 64, 2503 (1990)

12. Constantin, P.: Commun. Math. Phys. 129, 241 (1990)

13. Feller, W.: An introduction to probability theory and its applications, Vol. I, II, New York: John Wiley 1966

14. Forster, D., Nelson, D., Stephen, M.: Phys. Rev. A 16(2), $732-749$ (1977)

15. Glimm, J., Sharp, D. H.: Preprint, Los Alamos 1990

16. Helland, I. S.: Scand. J. Stat. 9, 79-94 (1982)

17. Hentschel, H. G. E., Procaccia, I.: Phys. Rev. A 29(3), 1461-1469 (1984)

18. Ibragimov, A. I.: Theory Prob. Applic. 7, 349-382 (1962)

19. Kesten, H., Papanicolaou, G. C.: Commun. Math. Phys. 65, 19-128 (1979)

20. Khasminski, R. Z.: Th. Prob. Appl. 5, 179-196 (1960)

21. Koch, D. L., Brody, J. Phys. of Fluids A 1, 47-51 (1989)

22. Kolmogorov, A. N.: Dokl. Acad. Nauka SSSR 31, 538-541 (1941); J. Fluid Mech. 12, 82-85 (1962)

23. Kozlov, S. M.: Russ. Math. Surv. $40(2), 73$ (1985)

24. Kraichnan, R.: Complex Syst. 1, 805-820 (1987)

25. Kraichnan, R.: Phys. Fluids 6, 575-598 (1987)

26. Lifshits, I. M., Gredeskul, S. A., Pastur, L. A.: Introduction to the theory of disordered systems. New York: Wiley-Interscience 1988

27. Lovejoy, S.: Science 216, 185 (1982)

28. Matheron, G., De Marsily, G.: Water Resources Res. 16, 901-907 (1980)

29. McComb, W. D.: The physics of fluid turbulence. Oxford Engr. Sci. Series vol. 25, Oxford: Clarendon Press 1990

30. McKean, H. P.: Stochastic integrals. New York: London: Academic Press 1969

31. Meneveau, C., Sreenivasan, K. R.: Phys. Rev. Lett. 59, 797 (1987)

32. Obukhov, A. M.: J. Fluid Mech. 12, 77-81 (1962)

33. Oelschlager, K.: Ann. Prob. 16, 1084-1126 (1988)

34. Orey, S.: Z. Warsch. Verb. Geb. 15, 249-256 (1970)

35. Orey, S.: Duke Math. J. 25, 543-546 (1958)

36. Osada, H.: In: Proc. 4th Japan-USSR Symp. Prob. Theory, Lect. Notes in Math. vol. 1021, pp. 507-517. Berlin, Heidelberg, New York: Springer 1983 
37. Papanicolaou, G. C., Varadhan, S. R. S.: In: Random Fields, Coll. Math. Soc. Janos Bolyai 27. Fritz. J., Leibowitz, J. L. (eds.). pp. 835-863. Amsterdam: North-Holland 1982

38. Richardson, L. F.: Proc. R. Soc. Lond. Ser. A 110, 709-737 (1926)

39. Roberts, P. H.: J. Fluid Mech. 11, 257-273 (1961)

40. Rose, H. A.: J. Fluid Mech. 81 (4), 719-734 (1977)

41. Williams, F.: Combustion theory. Menlo Park, CA: Benjamin Comming 1985

42. Yakhot, V., Orszag, S.: J. Sci. Comp. 1, 3-51 (1986)

43. Avellaneda, M., Torquato, S., Kim, I. C.: Physics of Fluids A 3(8), 1880-1891 (1991)

Communicated by S.-T. Yau 